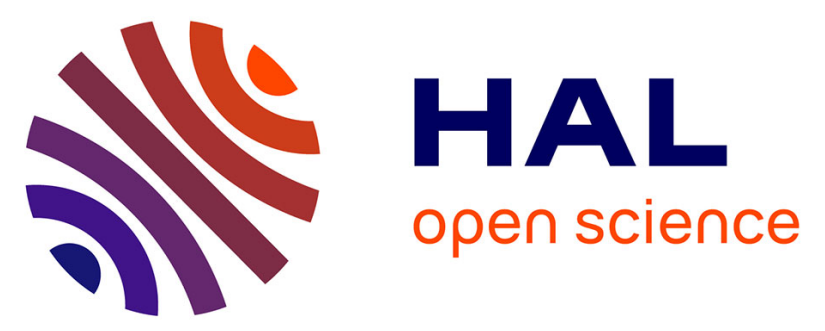

\title{
Asymptotic behavior for the Vlasov-Poisson equations with strong external magnetic field. Straight magnetic field lines
}

Mihai Bostan

\section{- To cite this version:}

Mihai Bostan. Asymptotic behavior for the Vlasov-Poisson equations with strong external magnetic field. Straight magnetic field lines. SIAM Journal on Mathematical Analysis, 2019, 51 (3), pp.27132747. 10.1137/18M122813X . hal-01683869v2

\section{HAL Id: hal-01683869 \\ https://hal.science/hal-01683869v2}

Submitted on 19 Aug 2019

HAL is a multi-disciplinary open access archive for the deposit and dissemination of scientific research documents, whether they are published or not. The documents may come from teaching and research institutions in France or abroad, or from public or private research centers.
L'archive ouverte pluridisciplinaire HAL, est destinée au dépôt et à la diffusion de documents scientifiques de niveau recherche, publiés ou non, émanant des établissements d'enseignement et de recherche français ou étrangers, des laboratoires publics ou privés. 


\title{
Asymptotic behavior for the Vlasov-Poisson equations with strong external magnetic field. Straight magnetic field lines
}

\author{
Mihaï BOSTAN *
}

(May 4, 2019)

\begin{abstract}
The subject matter of this paper concerns the Vlasov-Poisson equations in the framework of magnetic confinement. We study the behavior of the VlasovPoisson system with strong external magnetic field, when neglecting the curvature of the magnetic lines. The arguments rely on averaging techniques. We intend to determine second order approximations and to retrieve the usual electric cross field drift, the magnetic gradient drift.
\end{abstract}

Keywords: Vlasov-Poisson system, two-scale analysis, averaging, homogenization.

AMS classification: 35Q75, 78A35, 82D10.

\section{Introduction}

Let $f=f(t, x, v)$ be the phase space density of a population of charged particles of mass $m$, charge $q$, depending on time $t$, position $x$ and velocity $v$. Motivated by the magnetic confinement, we study the Vlasov-Poisson equations, with a strong external non vanishing magnetic field. Neglecting the curvature of the magnetic lines, we assume that the external magnetic field is orthogonal to $O x_{1}, O x_{2}$. In the two dimensional setting $x=\left(x_{1}, x_{2}\right), v=\left(v_{1}, v_{2}\right)$, the Vlasov equation writes

$$
\partial_{t} f^{\varepsilon}+v \cdot \nabla_{x} f^{\varepsilon}+\frac{q}{m}\left\{E\left[f^{\varepsilon}(t)\right](x)+B^{\varepsilon}(x)^{\perp} v\right\} \cdot \nabla_{v} f^{\varepsilon}=0, \quad(t, x, v) \in \mathbb{R}_{+} \times \mathbb{R}^{2} \times \mathbb{R}^{2} .
$$

Here the notation ${ }^{\perp}(\cdot)$ stands for the rotation of angle $-\pi / 2$, i.e., ${ }^{\perp} v=\mathcal{R}(-\pi / 2) v=$ $\left(v_{2},-v_{1}\right), v=\left(v_{1}, v_{2}\right) \in \mathbb{R}^{2}$ and the magnetic field writes $\mathbf{B}^{\varepsilon}(x)=\left(0,0, B^{\varepsilon}(x)\right)=$ $(0,0, B(x) / \varepsilon)$, where $B(x)$ is a given function and $\varepsilon>0$ is a small parameter related

*Aix Marseille Université, CNRS, Centrale Marseille, I2M, Marseille France, Centre de Mathématiques et Informatique, UMR 7373, 39 rue Frédéric Joliot Curie, 13453 Marseille Cedex 13 France. E-mail : mihai.bostan@univ-amu.fr. 
to the ratio between the cyclotronic period and the advection time scale. The electric field $E\left[f^{\varepsilon}(t)\right]=-\nabla_{x} \Phi\left[f^{\varepsilon}(t)\right]$ derives from the potential

$$
\Phi\left[f^{\varepsilon}(t)\right](x)=-\frac{q}{2 \pi \varepsilon_{0}} \int_{\mathbb{R}^{2}} \int_{\mathbb{R}^{2}} \ln \left|x-x^{\prime}\right| f^{\varepsilon}\left(t, x^{\prime}, v^{\prime}\right) \mathrm{d} v^{\prime} \mathrm{d} x^{\prime}
$$

satisfying the Poisson equation

$$
-\varepsilon_{0} \Delta_{x} \Phi\left[f^{\varepsilon}(t)\right]=q \int_{\mathbb{R}^{2}} f^{\varepsilon}(t, x, v) \mathrm{d} v, \quad(t, x) \in \mathbb{R}_{+} \times \mathbb{R}^{2}
$$

whose fundamental solution is $z \rightarrow-\frac{1}{2 \pi} \ln |z|, z \in \mathbb{R}^{2} \backslash\{0\}$. Here $\varepsilon_{0}$ represents the electric permittivity. For any particle density $f=f(x, v)$, the notation $E[f]$ stands for the Poisson electric field

$$
E[f](x)=\frac{q}{2 \pi \varepsilon_{0}} \int_{\mathbb{R}^{2}} \int_{\mathbb{R}^{2}} f\left(x^{\prime}, v^{\prime}\right) \frac{x-x^{\prime}}{\left|x-x^{\prime}\right|^{2}} \mathrm{~d} v^{\prime} \mathrm{d} x^{\prime}
$$

and $\rho[f], j[f]$ are the charge and current densities respectively

$$
\rho[f]=q \int_{\mathbb{R}^{2}} f(\cdot, v) \mathrm{d} v, \quad j[f]=q \int_{\mathbb{R}^{2}} f(\cdot, v) v \mathrm{~d} v .
$$

We complete the above system by the initial condition

$$
f^{\varepsilon}(0, x, v)=f_{\text {in }}(x, v), \quad(x, v) \in \mathbb{R}^{2} \times \mathbb{R}^{2} .
$$

We intend to investigate the asymptotic behavior of the problem (1), (3), (4) when $\varepsilon$ goes to 0 , by studying the well posedness of the limit models and establishing second error estimates. We refer to $[19,20,21,29,30,12,15,27,3,5,6,7]$ for previous results on this topic where, most of the time, the authors studied the case of uniform magnetic fields (see also $[17,22]$ for results with magnetic field of constant direction but variable strength and with magnetic field of constant strength but variable direction).

Solving numerically (1), (2), when $\varepsilon$ becomes small, requires a huge amount of computations. For example, when explicit numerical methods are used, CFL stability conditions apply, leading to a small time step of order $\varepsilon$. One alternative is to construct suitable numerical schemes, preserving the asymptotic cf. [16, 18, 14]. Here we have in mind another possibility. Instead of solving the problem (1), (2), which appears in a singular form, due to the large magnetic field, we are looking for a regular reformulation of it, whose numerical resolution is not penalized anymore by the smallness of the parameter $\varepsilon$. Certainly, the new problem will not be equivalent to the original one, but up to a second order term with respect to $\varepsilon$, the solutions will coincide. Therefore, when $\varepsilon$ becomes small we can obtain very good approximations for the Vlasov-Poisson system with large external magnetic field, with a numerical cost not depending on $\varepsilon$.

As usual, the starting point for such analysis is to look for quantities which have small time variations, like the guiding center, the rotation of the relative velocity with respect to the electric cross field drift $[17,27,30]$. Taking the average with respect to the fast cyclotronic motion, we obtain the model (6) for well prepared initial particle densities, which corresponds to the asymptotic model in [17] (where the authors also proposed asymptotically preserving particle-in-cell methods for solving it numerically). 
Its well posedness is established: a classical solution exists globally in time and it is unique. The main properties of this solution are detailed cf. Remark 4.1. For any $k \in \mathbb{N}$, the notation $C_{b}^{k}$ stands for $k$ times continuously differentiable functions, whose all partial derivatives, up to order $k$, are bounded.

\section{Theorem 1.1}

Consider a non negative, smooth, compactly supported initial particle density $\tilde{f}_{\text {in }} \in$ $C_{c}^{1}\left(\mathbb{R}^{2} \times \mathbb{R}^{2}\right)$ and a smooth magnetic field $B^{\varepsilon}=\frac{B}{\varepsilon} \in C_{b}^{2}\left(\mathbb{R}^{2}\right)$ such that $\inf _{x \in \mathbb{R}^{2}}\left|B^{\varepsilon}(x)\right|=$ $B_{0}^{\varepsilon}>0$ (that is $B_{0}^{\varepsilon}=\frac{B_{0}}{\varepsilon}$, inf $x \in \mathbb{R}^{2}|B(x)|=B_{0}>0$ ). There is a unique particle density $\tilde{f} \in C^{1}\left(\mathbb{R}_{+} \times \mathbb{R}^{2} \times \mathbb{R}^{2}\right)$ whose restriction on $[0, T] \times \mathbb{R}^{2} \times \mathbb{R}^{2}$ is compactly supported for any $T \in \mathbb{R}_{+}$, whose Poisson electric field belongs to $C^{1}\left(\mathbb{R}_{+} \times \mathbb{R}^{2}\right)$

$$
E[\tilde{f}(t)](x)=\frac{q}{2 \pi \varepsilon_{0}} \int_{\mathbb{R}^{2}} \int_{\mathbb{R}^{2}} \tilde{f}\left(t, x^{\prime}, \tilde{v}^{\prime}\right) \frac{x-x^{\prime}}{\left|x-x^{\prime}\right|^{2}} \mathrm{~d} \tilde{v}^{\prime} \mathrm{d} x^{\prime}, \quad(t, x) \in \mathbb{R}_{+} \times \mathbb{R}^{2}
$$

satisfying

$$
\begin{gathered}
\partial_{t} \tilde{f}+\left(\frac{{ }^{\perp} E[\tilde{f}(t)]}{B^{\varepsilon}}-\frac{m|\tilde{v}|^{2}}{2 q B^{\varepsilon}} \frac{{ }^{\perp} \nabla B^{\varepsilon}}{B^{\varepsilon}}\right) \cdot \nabla_{x} \tilde{f}+\frac{1}{2}\left(\frac{{ }^{\perp} E[\tilde{f}(t)]}{B^{\varepsilon}} \cdot \frac{\nabla_{x} B^{\varepsilon}}{B^{\varepsilon}}\right) \tilde{v} \cdot \nabla_{\tilde{v}} \tilde{f}=0 \\
\tilde{f}(0, x, \tilde{v})=\tilde{f}_{\text {in }}(x, \tilde{v}), \quad(x, \tilde{v}) \in \mathbb{R}^{2} \times \mathbb{R}^{2} .
\end{gathered}
$$

Moreover, if for some integer $k \geq 2$ we have $\tilde{f}_{\text {in }} \in C_{c}^{k}\left(\mathbb{R}^{2} \times \mathbb{R}^{2}\right), B^{\varepsilon} \in C^{k+1}\left(\mathbb{R}^{2}\right)$, then $\tilde{f} \in C^{k}\left(\mathbb{R}_{+} \times \mathbb{R}^{2} \times \mathbb{R}^{2}\right)$ and $E[\tilde{f}] \in C^{k}\left(\mathbb{R}_{+} \times \mathbb{R}^{2}\right)$.

It is easily seen that the three dimensional case, with magnetic field of fixed direction, comes similarly. Indeed, when the particle density $f^{\varepsilon}$ depends on $\left(t, x_{1}, x_{2}, x_{3}, v_{1}, v_{2}, v_{3}\right)$, the Vlasov equation (1) also contains the terms $v_{3} \partial_{x_{3}} f^{\varepsilon}+\frac{q}{m} E_{3}\left[f^{\varepsilon}(t)\right] \partial_{v_{3}} f^{\varepsilon}$ and the model (6) becomes

$$
\begin{aligned}
\partial_{t} \tilde{f}+v_{3} \partial_{x_{3}} \tilde{f} & +\frac{q}{m} E_{3}[\tilde{f}(t)] \partial_{v_{3}} \tilde{f}+\left(\frac{{ }^{\perp} E[\tilde{f}(t)]}{B^{\varepsilon}}-\frac{m|\tilde{v}|^{2}}{2 q B^{\varepsilon}} \frac{{ }^{\perp} \nabla B^{\varepsilon}}{B^{\varepsilon}}\right) \cdot \nabla_{x_{1}, x_{2}} \tilde{f} \\
& +\frac{1}{2}\left(\frac{{ }^{\perp} E[\tilde{f}(t)]}{B^{\varepsilon}} \cdot \frac{\nabla_{x} B^{\varepsilon}}{B^{\varepsilon}}\right) \tilde{v} \cdot \nabla_{\tilde{v}} \tilde{f}=0, \quad\left(t, x, \tilde{v}, v_{3}\right) \in \mathbb{R}_{+} \times \mathbb{R}^{3} \times \mathbb{R}^{3}
\end{aligned}
$$

where ${ }^{\perp} E=\left(E_{2},-E_{1}\right), \nabla B^{\varepsilon}=\left(\partial_{x_{1}} B^{\varepsilon}, \partial_{x_{2}} B^{\varepsilon}\right),{ }^{\perp} \nabla B^{\varepsilon}=\left(\partial_{x_{2}} B^{\varepsilon},-\partial_{x_{1}} B^{\varepsilon}\right)$. For more general results involving curved magnetic field lines, we refer to $[10,11]$, where the well posedness of these asymptotic regimes is investigated, together with the error estimates.

We concentrate on the error estimates, which is the most difficult part of this study, since we deal with non linear models, perturbed by stiff terms. The error estimates require a considerable effort : we need to construct a suitable corrector and to perform accurate balance computations, in order to cancel all the stiff terms. We prove that the solution of (6) approximates the solution of the Vlasov-Poisson system (1), (2) up to a second order term with respect to $\varepsilon$. We mention that most of the studies presents convergence results, without indicating error estimates. The main idea is to split the advection field of the Vlasov equation into a fast and slow dynamics, such that 
the guiding center is left (exactly) invariant by the fast dynamics. To the best of our knowledge this idea is new. The advantages are multiple. The fast dynamics becomes periodic (even for a non homogeneous magnetic field). Accordingly, the homogenization procedure comes easily, by averaging over one period, instead of taking ergodic means.

\section{Theorem 1.2}

Let $B \in C_{b}^{3}\left(\mathbb{R}^{2}\right)$ be a smooth magnetic field, such that $\inf _{x \in \mathbb{R}^{2}}|B(x)|=B_{0}>0$. Consider a family of non negative, smooth, uniformly compactly supported particle densities $\left(g^{\varepsilon}\right)_{\varepsilon>0} \subset C_{c}^{2}\left(\mathbb{R}^{2} \times \mathbb{R}^{2}\right)$

$\exists R_{\tilde{x}}, R_{\tilde{v}}>0: \operatorname{supp} g^{\varepsilon} \subset\left\{(\tilde{x}, \tilde{v}) \in \mathbb{R}^{2} \times \mathbb{R}^{2}:|\tilde{x}| \leq R_{\tilde{x}} \quad\right.$ and $\left.|\tilde{v}| \leq R_{\tilde{v}}\right\}, \quad \sup _{\varepsilon>0}\left\|g^{\varepsilon}\right\|_{C^{2}}<+\infty$.

We assume that the particle densities are well prepared i.e.,

$$
\sup _{\varepsilon>0} \frac{\left\|^{\perp} \tilde{v} \cdot \nabla_{\tilde{v}} g^{\varepsilon}\right\|_{L^{2}\left(\mathbb{R}^{2} \times \mathbb{R}^{2}\right)}}{\varepsilon^{2}}<+\infty .
$$

We denote by $\left(f^{\varepsilon}\right)_{\varepsilon>0}$ the solutions of the Vlasov-Poisson equations with external magnetic field (1), (2) corresponding to the initial conditions

$$
f^{\varepsilon}(0, x, v)=g^{\varepsilon}\left(x+\varepsilon \frac{{ }^{\perp} v}{\omega_{c}}, v-\varepsilon \frac{{ }^{\perp} E\left[g^{\varepsilon}\right]}{B}\right), \quad(x, v) \in \mathbb{R}^{2} \times \mathbb{R}^{2}, \quad \varepsilon>0 .
$$

Then for any $T \in \mathbb{R}_{+}$, there is $\varepsilon_{T}>0$ and $C_{T}>0$ such that for any $0<\varepsilon \leq \varepsilon_{T}$

$$
\sup _{t \in[0, T]}\left\{\int_{\mathbb{R}^{2}} \int_{\mathbb{R}^{2}}\left[f^{\varepsilon}(t, x, v)-\tilde{f}\left(t, x+\varepsilon \frac{{ }^{\perp} v}{\omega_{c}}, v-\varepsilon \frac{{ }^{\perp} E[\tilde{f}(t)]}{B}\right)\right]^{2} \mathrm{~d} v \mathrm{~d} x\right\}^{1 / 2} \leq C_{T} \varepsilon^{2}
$$

where $\tilde{f}$ is the solution of (6), (5) corresponding to the initial condition $\tilde{f}(0)=\left\langle g^{\varepsilon}\right\rangle$ (here the notation $\langle\cdot\rangle$ stands for the average along the characteristic flow of the vector field $\omega_{c}(x)^{\perp} v \cdot \nabla_{v}$, see Proposition 3.1).

Our paper is organized as follows. In Section 2 we discuss the well posedness of the Vlasov-Poisson problem with external magnetic field. The regular reformulation of the Vlasov-Poisson problem is derived by formal computations in Section 3. Its well posedness is established in Section 4. The error estimate, when the initial conditions are well prepared, is shown in Section 5 and relies on the construction of a corrector term. More general results, for initial conditions not necessarily well prepared, or the three dimensional setting with curved magnetic lines, are discussed in the last section. These extensions will be the topic of future works.

\section{Strong solutions for the Vlasov-Poisson problem with external magnetic field}

The well posedness of the Vlasov-Poisson problem is well known. We refer to [1] for weak solutions, and to [31, 24, 28] for strong solutions. Using essentially the same arguments, leads to global existence and uniqueness for the strong solution of the 
Vlasov-Poisson problem with external magnetic field. Moreover, we establish uniform estimates with respect to the magnetic field, which will be crucial when studying the asymptotic behavior with strong magnetic fields. More exactly we prove the following result, see Appendix A for the main lines of the proof.

\section{Theorem 2.1}

Consider a non negative, smooth, compactly supported initial particle density $f_{\text {in }} \in$ $C_{c}^{1}\left(\mathbb{R}^{2} \times \mathbb{R}^{2}\right)$ and a smooth magnetic field $B \in C_{b}^{1}\left(\mathbb{R}^{2}\right)$. There is a unique particle density $f \in C^{1}\left(\mathbb{R}_{+} \times \mathbb{R}^{2} \times \mathbb{R}^{2}\right)$, whose restriction on $[0, T] \times \mathbb{R}^{2} \times \mathbb{R}^{2}$ is compactly supported for any $T \in \mathbb{R}_{+}$, whose Poisson electric field is smooth $E[f] \in C^{1}\left(\mathbb{R}_{+} \times \mathbb{R}^{2}\right)$, satisfying

$$
\begin{gathered}
\partial_{t} f+v \cdot \nabla_{x} f+\frac{q}{m}\left(E[f(t)]+B^{\perp} v\right) \cdot \nabla_{v} f=0, \quad(t, x, v) \in \mathbb{R}_{+} \times \mathbb{R}^{2} \times \mathbb{R}^{2} \\
E[f(t)](x)=\frac{q}{2 \pi \varepsilon_{0}} \int_{\mathbb{R}^{2}} \int_{\mathbb{R}^{2}} f\left(t, x^{\prime}, v^{\prime}\right) \frac{x-x^{\prime}}{\left|x-x^{\prime}\right|^{2}} \mathrm{~d} v^{\prime} \mathrm{d} x^{\prime}, \quad(t, x) \in \mathbb{R}_{+} \times \mathbb{R}^{2} \\
f(0, x, v)=f_{\text {in }}(x, v), \quad(x, v) \in \mathbb{R}^{2} \times \mathbb{R}^{2} .
\end{gathered}
$$

Moreover, if for some integer $k \geq 2$ we have $f_{\text {in }} \in C_{c}^{k}\left(\mathbb{R}^{2} \times \mathbb{R}^{2}\right), B \in C_{b}^{k}\left(\mathbb{R}^{2}\right)$, then $f \in C^{k}\left(\mathbb{R} \times \mathbb{R}^{2} \times \mathbb{R}^{2}\right)$ and $E[f] \in C^{k}\left(\mathbb{R}_{+} \times \mathbb{R}^{2}\right)$.

\section{Remark 2.1}

1. The solution constructed in Theorem 2.1 satisfies the conservation of the particle number and total energy

$$
\frac{\mathrm{d}}{\mathrm{d} t} \int_{\mathbb{R}^{2}} \int_{\mathbb{R}^{2}} f(t, x, v) \mathrm{d} v \mathrm{~d} x=0, \quad t \in \mathbb{R}_{+}
$$

$\frac{\mathrm{d}}{\mathrm{d} t}\left\{\int_{\mathbb{R}^{2}} \int_{\mathbb{R}^{2}} \frac{m|v|^{2}}{2} f(t, x, v) \mathrm{d} v \mathrm{~d} x-\frac{1}{4 \pi \varepsilon_{0}} \int_{\mathbb{R}^{2}} \int_{\mathbb{R}^{2}} \rho[f(t)](x) \rho[f(t)]\left(x^{\prime}\right) \ln \left|x-x^{\prime}\right| \mathrm{d} x^{\prime} \mathrm{d} x\right\}=0$.

2. Notice also that we have the following balance for the total momentum

$$
\begin{gathered}
\frac{\mathrm{d}}{\mathrm{d} t} \int_{\mathbb{R}^{2}} \int_{\mathbb{R}^{2}} f(t, x, v) m v \mathrm{~d} v \mathrm{~d} x-q \int_{\mathbb{R}^{2}} \int_{\mathbb{R}^{2}} f(t, x, v) B(x)^{\perp} v \mathrm{~d} v \mathrm{~d} x=\int_{\mathbb{R}^{2}} \rho[f(t)] E[f(t)] \mathrm{d} x \\
=\varepsilon_{0} \int_{\mathbb{R}^{2}} \mathbf{1}_{\text {supp } \rho[f(t)]} \operatorname{div}_{x} E[f(t)] E[f(t)] \mathrm{d} x \\
=\varepsilon_{0} \int_{\mathbb{R}^{2}} \mathbf{1}_{\text {supp } \rho[f(t)]} \operatorname{div}_{x}\left(E[f(t)] \otimes E[f(t)]-\frac{|E[f(t)]|^{2}}{2} I_{2}\right) \mathrm{d} x=0 .
\end{gathered}
$$

When the magnetic field is uniform, we obtain

$$
\frac{\mathrm{d}}{\mathrm{d} t} \int_{\mathbb{R}^{2}} \int_{\mathbb{R}^{2}} f(t, x, v) m v \mathrm{~d} v \mathrm{~d} x=\frac{q B}{m} \perp\left(\int_{\mathbb{R}^{2}} \int_{\mathbb{R}^{2}} f(t, x, v) m v \mathrm{~d} v \mathrm{~d} x\right)
$$

saying that the total momentum rotates at the cyclotronic frequency $\omega_{c}=\frac{q B}{m}$

$$
\int_{\mathbb{R}^{2}} \int_{\mathbb{R}^{2}} f(t, x, v) m v \mathrm{~d} v \mathrm{~d} x=\mathcal{R}\left(-\omega_{c} t\right) \int_{\mathbb{R}^{2}} \int_{\mathbb{R}^{2}} f_{\text {in }}(x, v) m v \mathrm{~d} v \mathrm{~d} x, \quad t \in \mathbb{R}_{+} .
$$


3. By direct computation, when the magnetic field is uniform, we obtain

$$
\left[v \cdot \nabla_{x}+\frac{q}{m}\left(E[f]+B^{\perp} v\right) \cdot \nabla_{v}\right]\left(\frac{1}{2}\left|x+\frac{{ }^{\perp} v}{\omega_{c}}\right|^{2}-\frac{1}{2} \frac{|v|^{2}}{\omega_{c}^{2}}\right)=-\frac{E[f]}{B} \cdot{ }^{\perp} x .
$$

Therefore, after integration by parts, we deduce

$$
\begin{aligned}
\frac{\mathrm{d}}{\mathrm{d} t} \int_{\mathbb{R}^{2}} \int_{\mathbb{R}^{2}} f(t, x, v)\left(\frac{1}{2}\left|x+\frac{{ }^{\perp} v}{\omega_{c}}\right|^{2}-\frac{1}{2} \frac{|v|^{2}}{\omega_{c}^{2}}\right) \mathrm{d} v \mathrm{~d} x=-\int_{\mathbb{R}^{2}} \int_{\mathbb{R}^{2}} f \frac{E[f]}{B} \cdot{ }^{\perp} x \mathrm{~d} v \mathrm{~d} x \\
=-\frac{\varepsilon_{0}}{B q} \int_{\mathbb{R}^{2}} \mathbf{1}_{\text {supp } \rho[f(t)]} \operatorname{div}_{x} E[f(t)] E[f(t)] \cdot{ }^{\perp} x \mathrm{~d} x \\
=-\frac{\varepsilon_{0}}{B q} \int_{\mathbb{R}^{2}} \mathbf{1}_{\text {supp } \rho[f(t)]} \operatorname{div}_{x}\left(E[f(t)] \otimes E[f(t)]-\frac{|E[f(t)]|^{2}}{2} I_{2}\right) \cdot{ }^{\perp} x \mathrm{~d} x \\
=\frac{\varepsilon_{0}}{B q} \int_{\mathbb{R}^{2}} \mathbf{1}_{\text {supp } \rho[f(t)]}\left(E[f(t)] \otimes E[f(t)]-\frac{|E[f(t)]|^{2}}{2} I_{2}\right): \partial_{x}^{\perp} x \mathrm{~d} x=0 .
\end{aligned}
$$

\section{Asymptotic analysis by formal arguments}

We are interested on the asymptotic behavior of the particle densities $\left(f^{\varepsilon}\right)_{\varepsilon>0}$ satisfying (1), (2), (4) when $\varepsilon>0$ becomes small. We assume that the initial particle density and the external magnetic field $B^{\varepsilon}(x)=B(x) / \varepsilon$ are smooth

$$
f_{\text {in }} \geq 0, \quad f_{\text {in }} \in C_{c}^{1}\left(\mathbb{R}^{2} \times \mathbb{R}^{2}\right), \quad B \in C_{b}^{1}\left(\mathbb{R}^{2}\right) .
$$

Under the above assumptions, we know by Theorem 2.1 that for every $\varepsilon>0$, there is a unique strong solution $f^{\varepsilon} \in C^{1}\left(\mathbb{R}_{+} \times \mathbb{R}^{2} \times \mathbb{R}^{2}\right)$ (whose restriction on $[0, T] \times \mathbb{R}^{2} \times \mathbb{R}^{2}$ is compactly supported for any $\left.T \in \mathbb{R}_{+}\right), E^{\varepsilon}:=E\left[f^{\varepsilon}\right] \in C^{1}\left(\mathbb{R}_{+} \times \mathbb{R}^{2}\right)$ for the VlasovPoisson problem with external magnetic field $B^{\varepsilon}=B / \varepsilon$. By the arguments in the proof of Theorem 2.1 we also have uniform estimates with respect to $\varepsilon>0$ for the $L^{\infty}$ norm of the electric field $E^{\varepsilon}$ and the size of the support of the particle density $f^{\varepsilon}$. Let us denote by $\left(X^{\varepsilon}, V^{\varepsilon}\right)\left(t ; t_{0}, x, v\right)$ the characteristics associated to (1)

$$
\begin{gathered}
\frac{\mathrm{d} X^{\varepsilon}}{\mathrm{d} t}=V^{\varepsilon}\left(t ; t_{0}, x, v\right), \frac{\mathrm{d} V^{\varepsilon}}{\mathrm{d} t}=\frac{q}{m}\left[E^{\varepsilon}\left(t, X^{\varepsilon}\left(t ; t_{0}, x, v\right)\right)+\frac{B}{\varepsilon}\left(X^{\varepsilon}\left(t ; t_{0}, x, v\right)\right){ }^{\perp} V^{\varepsilon}\left(t ; t_{0}, x, v\right)\right] \\
X^{\varepsilon}\left(t ; t_{0}, x, v\right)=x, \quad V^{\varepsilon}\left(t ; t_{0}, x, v\right)=v .
\end{gathered}
$$

Clearly, the strong external magnetic field induces a large cyclotronic frequency with respect to the reciprocal advection time scale, and therefore a fast dynamics. Indeed, by introducing the characteristic scales $(\bar{t}, \bar{x}, \bar{v})$ for time, length, velocity, we have $\bar{t}=\bar{x} / \bar{v}$ and $\omega_{c}^{\varepsilon} \bar{t} \sim 1 / \varepsilon$. We use the notations $\omega_{c}^{\varepsilon}=q B^{\varepsilon} / m=\omega_{c} / \varepsilon, \omega_{c}=q B / m \sim 1 / \bar{t}$. The key point is to find out quantities which are left invariant with respect to the fast motion. It is well known that the guiding center, $X^{\varepsilon}(t)+\varepsilon{ }^{\perp} V^{\varepsilon}(t) / \omega_{c}\left(X^{\varepsilon}(t)\right)$ has small variations in time $[17,27,30]$. Another quantity having small variations is $\mathcal{R}\left(\int_{0}^{t} \frac{\omega_{c}\left(X^{\varepsilon}(\sigma)\right)}{\varepsilon} \mathrm{d} \sigma\right)\left[V^{\varepsilon}(t)-\varepsilon \frac{\perp E^{\varepsilon}\left(t, X^{\varepsilon}(t)\right)}{B\left(X^{\varepsilon}(t)\right)}\right]$, see $[23]$. 


\section{Lemma 3.1}

The following quantities

$$
X^{\varepsilon}(t)+\varepsilon^{\perp} V^{\varepsilon}(t) / \omega_{c}\left(X^{\varepsilon}(t)\right), \quad \mathcal{R}\left(\int_{0}^{t} \frac{\omega_{c}\left(X^{\varepsilon}(\sigma)\right)}{\varepsilon} \mathrm{d} \sigma\right)\left[V^{\varepsilon}(t)-\varepsilon \frac{{ }^{\perp} E^{\varepsilon}\left(t, X^{\varepsilon}(t)\right)}{B\left(X^{\varepsilon}(t)\right)}\right]
$$

have small variations in time.

\section{Proof.}

By direct computations we have

$$
\frac{\mathrm{d}}{\mathrm{d} t}\left[X^{\varepsilon}(t)+\varepsilon \frac{{ }^{\perp} V^{\varepsilon}(t)}{\omega_{c}\left(X^{\varepsilon}(t)\right)}\right]=\varepsilon\left[\frac{{ }^{\perp} E^{\varepsilon}\left(t, X^{\varepsilon}(t)\right)}{B\left(X^{\varepsilon}(t)\right)}-\frac{{ }^{\perp} V^{\varepsilon}(t) \otimes V^{\varepsilon}(t)}{\omega_{c}\left(X^{\varepsilon}(t)\right)^{2}} \nabla_{x} \omega_{c}\left(X^{\varepsilon}(t)\right)\right] .
$$

Notice also that

$$
\begin{aligned}
\frac{\mathrm{d}}{\mathrm{d} t}\left[V^{\varepsilon}(t)-\varepsilon \frac{{ }^{\perp} E^{\varepsilon}\left(t, X^{\varepsilon}(t)\right)}{B\left(X^{\varepsilon}(t)\right)}\right] & =\frac{\omega_{c}\left(X^{\varepsilon}(t)\right)}{\varepsilon} \perp\left[V^{\varepsilon}(t)-\varepsilon \frac{{ }^{\perp} E^{\varepsilon}\left(t, X^{\varepsilon}(t)\right)}{B\left(X^{\varepsilon}(t)\right)}\right] \\
& -\varepsilon \frac{\mathrm{d}}{\mathrm{d} t}\left[\frac{{ }^{\perp} E^{\varepsilon}\left(t, X^{\varepsilon}(t)\right)}{B\left(X^{\varepsilon}(t)\right)}\right]
\end{aligned}
$$

and therefore

$$
\begin{gathered}
\frac{\mathrm{d}}{\mathrm{d} t}\left\{\mathcal{R}\left(\int_{0}^{t} \frac{\omega_{c}\left(X^{\varepsilon}(\sigma)\right)}{\varepsilon} \mathrm{d} \sigma\right)\left[V^{\varepsilon}(t)-\varepsilon \frac{{ }^{\perp} E^{\varepsilon}\left(t, X^{\varepsilon}(t)\right)}{B\left(X^{\varepsilon}(t)\right)}\right]\right\} \\
=-\varepsilon \mathcal{R}\left(\int_{0}^{t} \frac{\omega_{c}\left(X^{\varepsilon}(\sigma)\right)}{\varepsilon} \mathrm{d} \sigma\right) \frac{\mathrm{d}}{\mathrm{d} t}\left[\frac{{ }^{\perp} E^{\varepsilon}\left(t, X^{\varepsilon}(t)\right)}{B\left(X^{\varepsilon}(t)\right)}\right] .
\end{gathered}
$$

Certainly, the above quantities are not exactly left invariant. The idea will be to split the advection field appearing in the Vlasov equation (1) into a fast and slow dynamics in such a way that the previous quantities become invariant. A consequence of this invariance is that the fast dynamics becomes periodic, which simplifies the averaging procedure. First of all, in order to simplify our computations, we perform a change of coordinates. Motivated by the calculation in (11), we introduce the relative velocity with respect to the electric cross field drift

$$
\tilde{v}=v-\varepsilon \frac{{ }^{\perp} E^{\varepsilon}(t, x)}{B(x)} .
$$

Accordingly, at any time $t \in \mathbb{R}_{+}$, we consider the new particle density

$$
\tilde{f}^{\varepsilon}(t, x, \tilde{v})=f^{\varepsilon}\left(t, x, \tilde{v}+\varepsilon \frac{{ }^{\perp} E\left[f^{\varepsilon}(t)\right](x)}{B(x)}\right), \quad(x, \tilde{v}) \in \mathbb{R}^{2} \times \mathbb{R}^{2} .
$$

Notice that this change of coordinates, which depends on the particle density through the electric field, does not change the charge density

$$
\rho\left[\tilde{f}^{\varepsilon}(t)\right]=q \int_{\mathbb{R}^{2}} \tilde{f}^{\varepsilon}(t, \cdot, \tilde{v}) \mathrm{d} \tilde{v}=q \int_{\mathbb{R}^{2}} f^{\varepsilon}(t, \cdot, v) \mathrm{d} v=\rho\left[f^{\varepsilon}(t)\right], \quad t \in \mathbb{R}_{+} .
$$


Therefore the Poisson electric fields corresponding to the particle densities $f^{\varepsilon}, \tilde{f}^{\varepsilon}$ coincide

$$
E\left[f^{\varepsilon}(t)\right]=E\left[\tilde{f}^{\varepsilon}(t)\right], \quad t \in \mathbb{R}_{+}
$$

and we can use the same notation $E^{\varepsilon}(t)$ for denoting them. For further use, see (16), notice that we have the following equality between the current densities $j\left[f^{\varepsilon}\right], j\left[\tilde{f}^{\varepsilon}\right]$

$$
\begin{gathered}
j\left[f^{\varepsilon}(t)\right]=q \int_{\mathbb{R}^{2}} f^{\varepsilon}(t, \cdot, v) v \mathrm{~d} v=q \int_{\mathbb{R}^{2}} \tilde{f}^{\varepsilon}(t, \cdot, \tilde{v})\left(\tilde{v}+\varepsilon \frac{{ }^{\perp} E^{\varepsilon}(t)}{B(x)}\right) \mathrm{d} \tilde{v} \\
=j\left[\tilde{f}^{\varepsilon}(t)\right]+\varepsilon \frac{{ }^{\varepsilon} E^{\varepsilon}(t)}{B(x)} \rho\left[\tilde{f}^{\varepsilon}(t)\right], \quad t \in \mathbb{R}_{+} .
\end{gathered}
$$

We add to (10) the hypothesis

$$
B_{0}:=\inf _{x \in \mathbb{R}^{2}}|B(x)|>0 \text { or equivalently } \omega_{0}:=\inf _{x \in \mathbb{R}^{2}}\left|\omega_{c}(x)\right|>0
$$

such that $(12),(13)$ are well defined. Observe that the new particle densities $\left(\tilde{f}^{\varepsilon}\right)_{\varepsilon>0}$ are smooth, $\tilde{f}^{\varepsilon} \in C^{1}\left(\mathbb{R}_{+} \times \mathbb{R}^{2} \times \mathbb{R}^{2}\right)$ and that the restrictions to $[0, T] \times \mathbb{R}^{2} \times \mathbb{R}^{2}$ are compactly supported, uniformly with respect to $\varepsilon \in] 0,1]$, for any $T \in \mathbb{R}_{+}$. The last statement comes from the similar property of the particle densities $\left(f^{\varepsilon}\right)_{\varepsilon>0}$, together with the uniform bound for the electric fields $\left(E^{\varepsilon}\right)_{\varepsilon>0}$ and the hypothesis (14). A straightforward computation leads to the following problem in the new coordinates $(x, \tilde{v})$

$$
\begin{gathered}
\partial_{t} \tilde{f}^{\varepsilon}+\left(\tilde{v}+\varepsilon \frac{{ }^{\perp} E^{\varepsilon}}{B}\right) \cdot \nabla_{x} \tilde{f}^{\varepsilon}-\varepsilon\left[\partial_{t}\left(\frac{{ }^{\perp} E^{\varepsilon}}{B}\right)+\partial_{x}\left(\frac{{ }^{\perp} E^{\varepsilon}}{B}\right)\left(\tilde{v}+\varepsilon \frac{{ }^{\perp} E^{\varepsilon}}{B}\right)\right] \cdot \nabla_{\tilde{v}} \tilde{f}^{\varepsilon} \\
+\frac{\omega_{c}(x)}{\varepsilon}{ }^{\perp} \tilde{v} \cdot \nabla_{\tilde{v}} \tilde{f}^{\varepsilon}=0, \quad(t, x, \tilde{v}) \in \mathbb{R}_{+} \times \mathbb{R}^{2} \times \mathbb{R}^{2} \\
\tilde{f}^{\varepsilon}(0, x, \tilde{v})=f_{\text {in }}\left(x, \tilde{v}+\varepsilon \frac{{ }^{\perp} E\left[f_{\text {in }}\right](x)}{B(x)}\right), \quad(x, \tilde{v}) \in \mathbb{R}^{2} \times \mathbb{R}^{2} .
\end{gathered}
$$

Notice that the time derivative of the electric field $E^{\varepsilon}$ can be written in terms of the particle density $f^{\varepsilon}$ (or $\tilde{f}^{\varepsilon}$ ). Indeed, thanks to the continuity equation

$$
\partial_{t} \rho\left[f^{\varepsilon}\right]+\operatorname{div}_{x} j\left[f^{\varepsilon}\right]=0
$$

we have

$$
\begin{aligned}
\partial_{t} E\left[f^{\varepsilon}\right] & =\frac{1}{2 \pi \varepsilon_{0}} \int_{\mathbb{R}^{2}} \partial_{t} \rho\left[f^{\varepsilon}(t)\right]\left(x-x^{\prime}\right) \frac{x^{\prime}}{\left|x^{\prime}\right|^{2}} \mathrm{~d} x^{\prime} \\
& =-\frac{1}{2 \pi \varepsilon_{0}} \int_{\mathbb{R}^{2}} \operatorname{div}_{x} j\left[f^{\varepsilon}\right]\left(x-x^{\prime}\right) \frac{x^{\prime}}{\left|x^{\prime}\right|^{2}} \mathrm{~d} x^{\prime} \\
& =-\frac{1}{2 \pi \varepsilon_{0}} \operatorname{div}_{x} \int_{\mathbb{R}^{2}} \frac{x^{\prime}}{\left|x^{\prime}\right|^{2}} \otimes j\left[f^{\varepsilon}(t)\right]\left(x-x^{\prime}\right) \mathrm{d} x^{\prime} \\
& =-\frac{1}{2 \pi \varepsilon_{0}} \operatorname{div}_{x} \int_{\mathbb{R}^{2}} \frac{x-x^{\prime}}{\left|x-x^{\prime}\right|^{2}} \otimes\left(j\left[\tilde{f}^{\varepsilon}(t)\right]\left(x^{\prime}\right)+\varepsilon \frac{{ }^{\perp} E^{\varepsilon}\left(t, x^{\prime}\right)}{B\left(x^{\prime}\right)} \rho\left[\tilde{f}^{\varepsilon}(t)\right]\left(x^{\prime}\right)\right) \mathrm{d} x^{\prime} .
\end{aligned}
$$

Finally the Vlasov equation (15) writes

$$
\partial_{t} \tilde{f}^{\varepsilon}+\varepsilon a^{\varepsilon}\left[\tilde{f}^{\varepsilon}(t)\right] \cdot \nabla_{x, \tilde{v}} \tilde{f}^{\varepsilon}+\frac{b^{\varepsilon}(x, \tilde{v})}{\varepsilon} \cdot \nabla_{x, \tilde{v}} \tilde{f}^{\varepsilon}=0, \quad(t, x, \tilde{v}) \in \mathbb{R}_{+} \times \mathbb{R}^{2} \times \mathbb{R}^{2}
$$


where $b^{\varepsilon} \cdot \nabla_{x, \tilde{v}}=\left(\varepsilon \tilde{v}+\varepsilon^{2} A_{x}^{\varepsilon}(x, \tilde{v})\right) \cdot \nabla_{x}+\omega_{c}(x)^{\perp} \tilde{v} \cdot \nabla_{\tilde{v}}$ and for any particle density $\tilde{f}$, $a^{\varepsilon}[\tilde{f}] \cdot \nabla_{x, \tilde{v}}$ stands for the vector field

$$
\begin{aligned}
a^{\varepsilon}[\tilde{f}] \cdot \nabla_{x, \tilde{v}} & =\left(\frac{{ }^{\perp} E[\tilde{f}]}{B}-A_{x}^{\varepsilon}\right) \cdot \nabla_{x}+\left[-\partial_{x}\left(\frac{{ }^{\perp} E[\tilde{f}]}{B}\right)\left(\tilde{v}+\varepsilon \frac{{ }^{\perp} E[\tilde{f}]}{B}\right)\right. \\
& \left.+\frac{1}{2 \pi \varepsilon_{0} B} \operatorname{div}_{x} \int_{\mathbb{R}^{2}} \frac{{ }^{\perp}\left(x-x^{\prime}\right)}{\left|x-x^{\prime}\right|^{2}} \otimes\left(j[\tilde{f}]+\varepsilon \frac{{ }^{\perp} E[\tilde{f}]}{B} \rho[\tilde{f}]\right)\left(x^{\prime}\right) \mathrm{d} x^{\prime}\right] \cdot \nabla_{\tilde{v}} .
\end{aligned}
$$

Here $A_{x}^{\varepsilon}(x, \tilde{v}) \cdot \nabla_{x}$ is a vector field, to be determined later on, not depending on the particle density $\tilde{f}$. We will distinguish between the fast dynamics along the vector field $\frac{b^{\varepsilon}}{\varepsilon} \cdot \nabla_{x, \tilde{v}}$ and the slow dynamics along the vector field $\varepsilon a^{\varepsilon} \cdot \nabla_{x, \tilde{v}}$. We pick the vector field $A_{x}^{\varepsilon}(x, \tilde{v}) \cdot \nabla_{x}$ entering the corrections in $a^{\varepsilon} \cdot \nabla_{x, \tilde{v}}$ and $b^{\varepsilon} \cdot \nabla_{x, \tilde{v}}$ such that $x+\varepsilon \frac{{ }^{\perp} \tilde{v}}{\omega_{c}(x)}$ is left invariant by the fast dynamics

$$
b^{\varepsilon} \cdot \nabla_{x, \tilde{v}}\left(x+\varepsilon \frac{\perp \tilde{v}}{\omega_{c}(x)}\right)=0
$$

that is

$$
\left(I_{2}-\varepsilon \frac{{ }^{\perp} \tilde{v} \otimes \nabla \omega_{c}}{\omega_{c}^{2}(x)}\right) A_{x}^{\varepsilon}(x, \tilde{v})=\frac{{ }^{\perp} \tilde{v} \otimes \tilde{v}}{\omega_{c}^{2}(x)} \nabla_{x} \omega_{c} .
$$

Obviously, when the magnetic field is uniform, i.e., $\nabla_{x} \omega_{c}=0$, there is no correction, $A_{x}^{\varepsilon}=0$. Notice that $A_{x}^{\varepsilon}$ is well defined for any $(x, \tilde{v})$ such that $\varepsilon \frac{{ }^{\perp} \tilde{v} \cdot \nabla \omega_{c}}{\omega_{c}^{2}(x)} \neq 1$, that is, for almost all $(x, \tilde{v}) \in \mathbb{R}^{2} \times \mathbb{R}^{2}$. By construction, the guiding center $x+\varepsilon \frac{{ }^{\perp} \tilde{v}}{\omega_{c}(x)}$ is left invariant by the fast dynamics along $\frac{b^{\varepsilon}}{\varepsilon} \cdot \nabla_{x, \tilde{v}}$ and it is easily seen that $|\tilde{v}|$ is left invariant as well. Therefore, any function which is left invariant by the fast dynamics, depends only on the guiding center $x+\varepsilon \frac{{ }^{\perp} \tilde{v}}{\omega_{c}(x)}$ and $|\tilde{v}|$.

Remark 3.1 The vector field in the Vlasov equation (17) is divergence free

$$
\operatorname{div}_{x, \tilde{v}}\left(\varepsilon a^{\varepsilon}[\tilde{f}]+\frac{b^{\varepsilon}}{\varepsilon}\right)=\varepsilon \operatorname{div}_{x}\left(\frac{{ }^{\perp} E[\tilde{f}]}{B}\right)-\varepsilon \operatorname{div}_{\tilde{v}}\left[\partial_{x}\left(\frac{{ }^{\perp} E[\tilde{f}]}{B}\right) \tilde{v}\right]=0 .
$$

Studying the asymptotic behavior of (17), when $\varepsilon$ goes to 0 reduces to averaging with respect to the flow of the fast dynamics generated by the advection field $\frac{b^{\varepsilon}(x, \tilde{v})}{\varepsilon} \cdot \nabla_{x, \tilde{v}} \mathrm{cf}$. $[3,5,8,6,7]$. Notice that, when the magnetic field is uniform, this characteristic flow is periodic, and therefore the averaging comes easily. This is the main reason why most of the mathematical studies concern uniform magnetic fields. Nevertheless, by taking into account the vector field $A_{x}^{\varepsilon} \cdot \nabla_{x}$, even when the magnetic field is non homogeneous, the characteristic flow associated to $b^{\varepsilon}(x, \tilde{v}) \cdot \nabla_{x, \tilde{v}}$ is periodic, which simplifies a lot the asymptotic analysis. Not only it allows us to derive formally the asymptotic regimes, but it will provide us a very useful tool for performing the error analysis. In particular, in the periodic setting it is possible to construct explicitly the corrector (and therefore to check its smoothness) that we need when establishing the error estimate. 
Proposition 3.1 We denote by $\left(X^{\varepsilon}(s ; x, \tilde{v}), \tilde{\mathcal{V}}^{\varepsilon}(s ; x, \tilde{v})\right)$ the characteristic flow of the autonomous vector field $b^{\varepsilon}(x, \tilde{v}) \cdot \nabla_{x, \tilde{v}}$

$$
\begin{gathered}
\frac{\mathrm{d} X^{\varepsilon}}{\mathrm{d} s}=\varepsilon \tilde{\mathcal{V}}^{\varepsilon}(s ; x, \tilde{v})+\varepsilon^{2} A_{x}^{\varepsilon}\left(X^{\varepsilon}(s ; x, \tilde{v}), \tilde{\mathcal{V}}^{\varepsilon}(s ; x, \tilde{v})\right), \frac{\mathrm{d} \tilde{\mathcal{V}}^{\varepsilon}}{\mathrm{d} s}=\omega_{c}\left(X^{\varepsilon}(s ; x, \tilde{v})\right){ }^{\perp} \tilde{\mathcal{V}}^{\varepsilon}(s ; x, \tilde{v}) \\
X^{\varepsilon}(0 ; x, \tilde{v})=x, \quad \tilde{\mathcal{V}}^{\varepsilon}(0 ; x, \tilde{v})=\tilde{v}
\end{gathered}
$$

and by $(X(s ; x, \tilde{v}), \tilde{\mathcal{V}}(s ; x, \tilde{v}))$ the characteristic flow of the autonomous vector field $b(x, \tilde{v}) \cdot \nabla_{x, \tilde{v}}=\omega_{c}(x)^{\perp} \tilde{v} \cdot \nabla_{\tilde{v}}$

$$
\frac{\mathrm{d} X}{\mathrm{~d} s}=0, \quad \frac{\mathrm{d} \tilde{\mathcal{V}}}{\mathrm{d} s}=\omega_{c}(X(s ; x, \tilde{v}))^{\perp} \tilde{\mathcal{V}}(s ; x, \tilde{v}), \quad X(0 ; x, \tilde{v})=x, \quad \tilde{\mathcal{V}}(0 ; x, \tilde{v})=\tilde{v}
$$

1. For $\varepsilon>0$ small enough, the flow $\left(X^{\varepsilon}, \tilde{V}^{\varepsilon}\right)$ is periodic. More exactly for any $(x, \tilde{v}) \in \mathbb{R}^{2} \times \mathbb{R}^{2}$ and $\varepsilon>0$ such that $\varepsilon \frac{|\tilde{v}|\left\|\nabla \omega_{c}\right\|_{L^{\infty}}}{\omega_{0}^{2}}<1$, the characteristic $s \rightarrow$ $\left(X^{\varepsilon}, \tilde{V}^{\varepsilon}\right)(s ; x, \tilde{v})$ is periodic, with smallest period $S^{\varepsilon}(x, \tilde{v})>0$.

2. For any $(x, \tilde{v}) \in \mathbb{R}^{2} \times \mathbb{R}^{2}$ and $\varepsilon>0$ such that $\varepsilon \frac{|\tilde{v}|\left\|\nabla \omega_{c}\right\|_{L} \infty}{\omega_{0}^{2}} \leq \frac{1}{2}$ we have

$$
\begin{gathered}
\left|X^{\varepsilon}(s ; x, \tilde{v})-X(s ; x, \tilde{v})\right|=\left|X^{\varepsilon}(s ; x, \tilde{v})-x\right| \leq \varepsilon \frac{4 \pi}{\omega_{0}}|\tilde{v}|, \quad s \in \mathbb{R} \\
S^{\varepsilon}(x, \tilde{v}) \leq \frac{2 \pi}{\omega_{0}}, \quad\left|S^{\varepsilon}(x, \tilde{v})-S(x, \tilde{v})\right| \leq \varepsilon\left\|\nabla \omega_{c}\right\|_{L^{\infty}} \frac{8 \pi^{2}|\tilde{v}|}{\omega_{0}^{3}}, \quad S(x, \tilde{v})=\frac{2 \pi}{\left|\omega_{c}(x)\right|} \leq \frac{2 \pi}{\omega_{0}}
\end{gathered}
$$

and

$$
\left|\tilde{\mathcal{V}}^{\varepsilon}(s ; x, \tilde{v})-\tilde{\mathcal{V}}(s ; x, \tilde{v})\right|=\left|\tilde{\mathcal{V}}^{\varepsilon}(s ; x, \tilde{v})-\mathcal{R}\left(-s \omega_{c}(x)\right) \tilde{v}\right| \leq \varepsilon\left\|\nabla \omega_{c}\right\|_{L^{\infty}} \frac{8 \pi^{2}}{\omega_{0}^{2}}|\tilde{v}|^{2}, \quad s \in\left[0, \frac{2 \pi}{\omega_{0}}\right] .
$$

3. For any continuous function $u \in C\left(\mathbb{R}^{2} \times \mathbb{R}^{2}\right)$ we define the averages along the flows of $b \cdot \nabla_{x, \tilde{v}}, b^{\varepsilon} \cdot \nabla_{x, \tilde{v}}$

$$
\begin{gathered}
\langle u\rangle(x, \tilde{v})=\frac{1}{S(x, \tilde{v})} \int_{0}^{S(x, \tilde{v})} u(X(s ; x, \tilde{v}), \tilde{\mathcal{V}}(s ; x, \tilde{v})) \mathrm{d} s, \quad(x, \tilde{v}) \in \mathbb{R}^{2} \times \mathbb{R}^{2} \\
\langle u\rangle_{\varepsilon}(x, \tilde{v})=\frac{1}{S^{\varepsilon}(x, \tilde{v})} \int_{0}^{S^{\varepsilon}(x, \tilde{v})} u\left(X^{\varepsilon}(s ; x, \tilde{v}), \tilde{\mathcal{V}}^{\varepsilon}(s ; x, \tilde{v})\right) \mathrm{d} s, \quad(x, \tilde{v}) \in \mathbb{R}^{2} \times \mathbb{R}^{2} .
\end{gathered}
$$

For any $R_{x}, R_{\tilde{v}} \in \mathbb{R}_{+}$we have

$$
\begin{gathered}
\|\langle u\rangle\|_{L^{\infty}\left(B\left(R_{x}\right) \times B\left(R_{\tilde{v}}\right)\right)} \leq\|u\|_{L^{\infty}\left(B\left(R_{x}\right) \times B\left(R_{\tilde{v}}\right)\right)} \\
\left\|\langle u\rangle_{\varepsilon}\right\|_{L^{\infty}\left(B\left(R_{x}\right) \times B\left(R_{\tilde{v}}\right)\right)} \leq\|u\|_{L^{\infty}\left(B\left(R_{x}^{\varepsilon}\right) \times B\left(R_{\tilde{v}}\right)\right)}, \quad R_{x}^{\varepsilon}=R_{x}+2 \varepsilon R_{\tilde{v}} / \omega_{0}
\end{gathered}
$$

where $B(R)$ stands for the closed ball of radius $R$ in $\mathbb{R}^{2}$.

4. If $u$ is Lipschitz continuous, then for any $(x, \tilde{v}) \in \mathbb{R}^{2} \times \mathbb{R}^{2}$ and $\varepsilon>0$ such that $\varepsilon \frac{|\tilde{v}| \mid\left\|\nabla \omega_{c}\right\|_{L} \infty}{\omega_{0}^{2}} \leq \frac{1}{2}$ we have

$$
\begin{aligned}
\frac{\left|\langle u\rangle_{\varepsilon}(x, \tilde{v})-\langle u\rangle(x, \tilde{v})\right|}{\varepsilon} & \leq \operatorname{Lip}(u) \frac{4 \pi}{\omega_{0}}|\tilde{v}|\left[1+\left\|\nabla \omega_{c}\right\|_{L^{\infty}} \frac{2 \pi}{\omega_{0}}|\tilde{v}|\right] \\
& +\sup _{\left|\tilde{v}^{\prime}\right|=|\tilde{v}|}\left|u\left(x, \tilde{v}^{\prime}\right)\right||| \nabla \omega_{c} \|_{L^{\infty}} \frac{8 \pi}{\omega_{0}^{2}}|\tilde{v}| .
\end{aligned}
$$


5. For any function $u \in C_{c}^{1}\left(\mathbb{R}^{2} \times \mathbb{R}^{2}\right)$ we have the inequality

$$
\|u-\langle u\rangle\|_{L^{2}\left(\mathbb{R}^{2} \times \mathbb{R}^{2}\right)} \leq \frac{2 \pi}{\omega_{0}}\left\|b \cdot \nabla_{x, \tilde{v}} u\right\|_{L^{2}\left(\mathbb{R}^{2} \times \mathbb{R}^{2}\right)} .
$$

6. For any function $u \in C^{1}\left(\mathbb{R}^{2} \times \mathbb{R}^{2}\right)$, we have $\langle u\rangle \in C^{1}\left(\mathbb{R}^{2} \times \mathbb{R}^{2}\right)$ and

$$
\left\langle\nabla_{x} u\right\rangle=\nabla_{x}\langle u\rangle, \quad \tilde{v} \cdot \nabla_{\tilde{v}}\langle u\rangle=\left\langle\tilde{v} \cdot \nabla_{\tilde{v}} u\right\rangle,{ }^{\perp} \tilde{v} \cdot \nabla_{\tilde{v}}\langle u\rangle=0 .
$$

\section{Proof.}

1. For any $s \in \mathbb{R}$ we have

$$
\tilde{\mathcal{V}}^{\varepsilon}(s)=\mathcal{R}\left(-\int_{0}^{s} \omega_{c}\left(X^{\varepsilon}(\sigma)\right) \mathrm{d} \sigma\right) \tilde{v}
$$

and therefore $\left|\tilde{\mathcal{V}}^{\varepsilon}(s)\right|=|\tilde{v}|$. By hypothesis (14) we know that $\omega_{c}$ has constant sign, and there is a unique $S^{\varepsilon}(x, \tilde{v})>0$ such that

$$
\operatorname{sgn} \omega_{c} \int_{0}^{S^{\varepsilon}(x, \tilde{v})} \omega_{c}\left(X^{\varepsilon}(\sigma ; x, \tilde{v})\right) \mathrm{d} \sigma=\int_{0}^{S^{\varepsilon}(x, \tilde{v})}\left|\omega_{c}\left(X^{\varepsilon}(\sigma ; x, \tilde{v})\right)\right| \mathrm{d} \sigma=2 \pi .
$$

Clearly we have $\tilde{\mathcal{V}}^{\varepsilon}\left(S^{\varepsilon}(x, \tilde{v}) ; x, \tilde{v}\right)=\tilde{v}$ and we claim that, for small $\varepsilon$, we also have $X^{\varepsilon}\left(S^{\varepsilon}(x, \tilde{v}) ; x, \tilde{v}\right)=x$ saying that the flow $\left(X^{\varepsilon}(s ; x, \tilde{v}), \tilde{\mathcal{V}}^{\varepsilon}(s ; x, \tilde{v})\right)$ is $S^{\varepsilon}(x, \tilde{v})$ periodic. The main ingredient when justifying this periodicity is the invariance of the guiding center, see the definition of the vector field $A_{x}^{\varepsilon}(x, \tilde{v}) \cdot \nabla_{x}$

$$
X^{\varepsilon}\left(S^{\varepsilon}\right)+\varepsilon \frac{{ }^{\perp} \tilde{\mathcal{V}}^{\varepsilon}\left(S^{\varepsilon}\right)}{\omega_{c}\left(X^{\varepsilon}\left(S^{\varepsilon}\right)\right)}=x+\varepsilon \frac{{ }^{\perp} \tilde{v}}{\omega_{c}(x)}
$$

we deduce

$$
\left|X^{\varepsilon}\left(S^{\varepsilon}\right)-x\right| \leq \varepsilon \frac{|\tilde{v}|}{\omega_{0}^{2}}\left|\omega_{c}\left(X^{\varepsilon}\left(S^{\varepsilon}\right)\right)-\omega_{c}(x)\right| \leq \frac{\varepsilon|\tilde{v}|\left\|\nabla \omega_{c}\right\|_{L^{\infty}}}{\omega_{0}^{2}}\left|X^{\varepsilon}\left(S^{\varepsilon}\right)-x\right| .
$$

Therefore, if $\frac{\varepsilon|\tilde{v}|\left\|\nabla \omega_{c}\right\|_{L} \infty}{\omega_{0}^{2}}<1$, we obtain $X^{\varepsilon}\left(S^{\varepsilon}(x, \tilde{v})\right)=x$.

2. Notice that for any $s \in \mathbb{R}$

$$
\begin{aligned}
\operatorname{det}\left(I_{2}-\varepsilon \frac{\perp \tilde{\mathcal{V}}^{\varepsilon}(s) \otimes \nabla \omega_{c}\left(X^{\varepsilon}(s)\right)}{\omega_{c}^{2}\left(X^{\varepsilon}(s)\right)}\right) & =1-\varepsilon \frac{{ }^{\perp} \tilde{\mathcal{V}}^{\varepsilon}(s) \cdot \nabla \omega_{c}\left(X^{\varepsilon}(s)\right)}{\omega_{c}^{2}\left(X^{\varepsilon}(s)\right)} \\
& \geq 1-\varepsilon \frac{\left|\tilde{\mathcal{V}}^{\varepsilon}(s)\right|\left|\nabla \omega_{c}\left(X^{\varepsilon}(s)\right)\right|}{\omega_{c}^{2}\left(X^{\varepsilon}(s)\right)} \\
& \geq 1-\frac{\varepsilon|\tilde{v}|\left\|\nabla \omega_{c}\right\|_{L^{\infty}}}{\omega_{0}^{2}} \geq \frac{1}{2}
\end{aligned}
$$

and therefore $A_{x}^{\varepsilon}\left(X^{\varepsilon}(s), \tilde{\mathcal{V}}^{\varepsilon}(s)\right)$ is well defined for any $s \in \mathbb{R}$. Moreover (18) implies

$$
\begin{aligned}
\left|A_{x}^{\varepsilon}\left(X^{\varepsilon}(s), \tilde{\mathcal{V}}^{\varepsilon}(s)\right)\right| & \leq \frac{\left|\tilde{\mathcal{V}}^{\varepsilon}(s)\right|^{2}}{\omega_{c}^{2}\left(X^{\varepsilon}(s)\right)}\left|\nabla \omega_{c}\left(X^{\varepsilon}(s)\right)\right|+\varepsilon \frac{\left|\tilde{\mathcal{V}}^{\varepsilon}(s)\right|\left|\nabla \omega_{c}\left(X^{\varepsilon}(s)\right)\right|}{\omega_{c}^{2}\left(X^{\varepsilon}(s)\right)}\left|A_{x}^{\varepsilon}\left(X^{\varepsilon}(s), \tilde{\mathcal{V}}^{\varepsilon}(s)\right)\right| \\
& \leq \frac{|\tilde{v}|^{2}}{\omega_{0}^{2}}\left\|\nabla \omega_{c}\right\|_{L^{\infty}}+\frac{1}{2}\left|A_{x}^{\varepsilon}\left(X^{\varepsilon}(s), \tilde{\mathcal{V}}^{\varepsilon}(s)\right)\right|
\end{aligned}
$$


and thus

$$
\varepsilon\left|A_{x}^{\varepsilon}\left(X^{\varepsilon}(s), \tilde{\mathcal{V}}^{\varepsilon}(s)\right)\right| \leq 2 \frac{\varepsilon|\tilde{v}|^{2}\left\|\nabla \omega_{c}\right\|_{L^{\infty}}}{\omega_{0}^{2}} \leq|\tilde{v}|
$$

By the previous point we know that $s \rightarrow X^{\varepsilon}(s ; x, \tilde{v})$ is $S^{\varepsilon}(x, \tilde{v})$ periodic, where

$$
S^{\varepsilon}(x, \tilde{v}) \leq \frac{1}{\omega_{0}} \int_{0}^{S^{\varepsilon}(x, \tilde{v})}\left|\omega_{c}\left(X^{\varepsilon}(\sigma)\right)\right| \mathrm{d} \sigma=\frac{2 \pi}{\omega_{0}} .
$$

Finally we write for any $s \in\left[0, S^{\varepsilon}(x, \tilde{v})\right] \subset\left[0,2 \pi / \omega_{0}\right]$

$$
\begin{aligned}
\left|X^{\varepsilon}(s ; x, \tilde{v})-x\right| & =\left|\int_{0}^{s}\left\{\varepsilon \tilde{\mathcal{V}}^{\varepsilon}(\sigma)+\varepsilon^{2} A_{x}^{\varepsilon}\left(X^{\varepsilon}(\sigma), \tilde{\mathcal{V}}^{\varepsilon}(\sigma)\right)\right\} \mathrm{d} \sigma\right| \\
& \leq 2 \varepsilon S^{\varepsilon}(x, \tilde{v})|\tilde{v}| \leq \varepsilon \frac{4 \pi}{\omega_{0}}|\tilde{v}| .
\end{aligned}
$$

By periodicity we have

$$
\left|X^{\varepsilon}(s ; x, \tilde{v})-X(s ; x, \tilde{v})\right|=\left|X^{\varepsilon}(s ; x, \tilde{v})-x\right| \leq \varepsilon \frac{4 \pi}{\omega_{0}}|\tilde{v}|, \quad s \in \mathbb{R} .
$$

Let us compare now $S^{\varepsilon}(x, \tilde{v})$ to $S(x, \tilde{v})=2 \pi /\left|\omega_{c}(x)\right|$, which is the smallest period of the flow $(X, \tilde{\mathcal{V}})$. Integrating over $\left[0, S^{\varepsilon}(x, \tilde{v})\right]$ the inequality

$$
\left|\omega_{c}(x)\right|-\left\|\nabla \omega_{c}\right\|_{L^{\infty}} \frac{\varepsilon 4 \pi|\tilde{v}|}{\omega_{0}} \leq \mid \omega_{c}\left(X^{\varepsilon}(\sigma)|\leq| \omega_{c}(x) \mid+\left\|\nabla \omega_{c}\right\|_{L^{\infty}} \frac{\varepsilon 4 \pi|\tilde{v}|}{\omega_{0}}\right.
$$

one gets

$$
S^{\varepsilon}(x, \tilde{v})\left(\left|\omega_{c}(x)\right|-\left\|\nabla \omega_{c}\right\|_{L^{\infty}} \frac{\varepsilon 4 \pi|\tilde{v}|}{\omega_{0}}\right) \leq 2 \pi \leq S^{\varepsilon}(x, \tilde{v})\left(\left|\omega_{c}(x)\right|+\left\|\nabla \omega_{c}\right\|_{L^{\infty}} \frac{\varepsilon 4 \pi|\tilde{v}|}{\omega_{0}}\right)
$$

implying that

$$
\left|\frac{1}{S^{\varepsilon}(x, \tilde{v})}-\frac{1}{S(x, \tilde{v})}\right| \leq \varepsilon\left\|\nabla \omega_{c}\right\|_{L^{\infty}} \frac{2|\tilde{v}|}{\omega_{0}}
$$

and

$$
\left|S^{\varepsilon}(x, \tilde{v})-S(x, \tilde{v})\right|=S^{\varepsilon}(x, \tilde{v}) S(x, \tilde{v})\left|\frac{1}{S^{\varepsilon}(x, \tilde{v})}-\frac{1}{S(x, \tilde{v})}\right| \leq \varepsilon\left\|\nabla \omega_{c}\right\|_{L^{\infty}} \frac{8 \pi^{2}|\tilde{v}|}{\omega_{0}^{3}} .
$$

Thanks to the inequality $\left\|\mathcal{R}(\theta)-\mathcal{R}\left(\theta^{\prime}\right)\right\| \leq\left|\theta-\theta^{\prime}\right|, \theta, \theta^{\prime} \in \mathbb{R}$, we obtain for any $s \in\left[0,2 \pi / \omega_{0}\right]$

$$
\begin{aligned}
\left|\tilde{\mathcal{V}}^{\varepsilon}(s ; x, \tilde{v})-\tilde{\mathcal{V}}(s ; x, \tilde{v})\right| & =\left|\mathcal{R}\left(-\int_{0}^{s} \omega_{c}\left(X^{\varepsilon}(\sigma)\right) \mathrm{d} \sigma\right) \tilde{v}-\mathcal{R}\left(-\int_{0}^{s} \omega_{c}(X(\sigma)) \mathrm{d} \sigma\right) \tilde{v}\right| \\
& \leq \int_{0}^{s}\left|\omega_{c}\left(X^{\varepsilon}(\sigma)\right)-\omega_{c}(X(\sigma))\right| \mathrm{d} \sigma|\tilde{v}| \\
& \leq \varepsilon\left\|\nabla \omega_{c}\right\|_{L^{\infty}} \frac{8 \pi^{2}}{\omega_{0}^{2}}|\tilde{v}|^{2} .
\end{aligned}
$$


3. The first inequality is obvious, sice $X(s ; x, \tilde{v})=x$ and $|\tilde{\mathcal{V}}(s ; x, \tilde{v})|=|\tilde{v}|$, for any $(s, x, \tilde{v}) \in \mathbb{R} \times \mathbb{R}^{2} \times \mathbb{R}^{2}$. For the second one use $|\tilde{\mathcal{V}} \varepsilon(s ; x, \tilde{v})|=|\tilde{v}|$ and notice that by the invariance of the center

$$
X^{\varepsilon}(s ; x, \tilde{v})+\varepsilon \frac{{ }^{\perp} \tilde{\mathcal{V}}^{\varepsilon}(s ; x, \tilde{v})}{\omega_{c}\left(X^{\varepsilon}(s ; x, \tilde{v})\right)}=x+\varepsilon \frac{{ }^{\perp} \tilde{v}}{\omega_{c}(x)}
$$

we have

$$
\left|X^{\varepsilon}(s ; x, \tilde{v})\right| \leq|x|+\left|X^{\varepsilon}(s ; x, \tilde{v})-x\right| \leq|x|+2 \varepsilon \frac{|\tilde{v}|}{\omega_{0}}, \quad(s, x, \tilde{v}) \in \mathbb{R} \times \mathbb{R}^{2} \times \mathbb{R}^{2} .
$$

4. When analyzing the asymptotic regimes with $\varepsilon \searrow 0$, it is worth noticing that the average $\langle\cdot\rangle_{\varepsilon}$ converges toward the average $\langle\cdot\rangle$. It means that, although the average $\langle\cdot\rangle_{\varepsilon}$ is not explicit (because we do not have formulae for the flow $\left(X^{\varepsilon}, \tilde{V}^{\varepsilon}\right)$ ), its contribution in the limit model can be determined by averaging along the flow $(X, \tilde{\mathcal{V}})$, which is completely explicit. Based on the previous statements, we have

$$
\begin{aligned}
& \left|\langle u\rangle_{\varepsilon}(x, \tilde{v})-\langle u\rangle(x, \tilde{v})\right| \leq \frac{1}{S^{\varepsilon}(x, \tilde{v})} \int_{0}^{S^{\varepsilon}(x, \tilde{v})}\left|u\left(X^{\varepsilon}(s), \tilde{\mathcal{V}}^{\varepsilon}(s)\right)-u(X(s), \tilde{\mathcal{V}}(s))\right| \mathrm{d} s \\
& +\left|\frac{1}{S^{\varepsilon}(x, \tilde{v})}-\frac{1}{S(x, \tilde{v})}\right| \int_{0}^{S^{\varepsilon}(x, \tilde{v})}|u(X(s), \tilde{\mathcal{V}}(s))| \mathrm{d} s+\frac{1}{S(x, \tilde{v})}\left|\int_{S(x, \tilde{v})}^{S^{\varepsilon}(x, \tilde{v})}\right| u(X(s), \tilde{\mathcal{V}}(s))|\mathrm{d} s| \\
& \leq \operatorname{Lip}(u) \sup _{0 \leq s \leq 2 \pi / \omega_{0}}\left(\left|X^{\varepsilon}(s)-X(s)\right|+\left|\tilde{\mathcal{V}}^{\varepsilon}(s)-\tilde{\mathcal{V}}(s)\right|\right) \\
& +\left[\left|\frac{1}{S^{\varepsilon}(x, \tilde{v})}-\frac{1}{S(x, \tilde{v})}\right| S^{\varepsilon}(x, \tilde{v})+\frac{\left|S^{\varepsilon}(x, \tilde{v})-S(x, \tilde{v})\right|}{S(x, \tilde{v})}\right] \sup _{\left|\tilde{v}^{\prime}\right|=|\tilde{v}|}\left|u\left(x, \tilde{v}^{\prime}\right)\right| \\
& \leq \varepsilon \operatorname{Lip}(u) \frac{4 \pi}{\omega_{0}}|\tilde{v}|\left[1+\left\|\nabla \omega_{c}\right\|_{L^{\infty}} \frac{2 \pi}{\omega_{0}}|\tilde{v}|\right]+\varepsilon \sup _{\left|\tilde{v}^{\prime}\right|=|\tilde{v}|}\left|u\left(x, \tilde{v}^{\prime}\right)\right|\left|\nabla \omega_{c} \|_{L^{\infty}} \frac{8 \pi}{\omega_{0}^{2}}\right| \tilde{v} \mid .
\end{aligned}
$$

5. We can write for any $(x, \tilde{v}) \in \mathbb{R}^{2} \times \mathbb{R}^{2}$

$$
\begin{aligned}
\langle u\rangle(x, \tilde{v})-u(x, \tilde{v}) & =\frac{1}{S(x, \tilde{v})} \int_{0}^{S(x, \tilde{v})}[u(X(s ; x, \tilde{v}), \tilde{\mathcal{V}}(s ; x, \tilde{v}))-u(x, \tilde{v})] \mathrm{d} s \\
& =\frac{1}{S(x, \tilde{v})} \int_{0}^{S(x, \tilde{v})} \int_{0}^{s} \frac{\mathrm{d}}{\mathrm{d} \sigma} u(X(\sigma ; x, \tilde{v}), \tilde{\mathcal{V}}(\sigma ; x, \tilde{v})) \mathrm{d} \sigma \mathrm{d} s \\
& =\frac{1}{S(x, \tilde{v})} \int_{0}^{S(x, \tilde{v})} \int_{0}^{s}(b \cdot \nabla u)(X(\sigma ; x, \tilde{v}), \tilde{\mathcal{V}}(\sigma ; x, \tilde{v})) \mathrm{d} \sigma \mathrm{d} s \\
& =\frac{1}{2 \pi} \int_{0}^{2 \pi} \int_{0}^{\frac{\theta}{\omega_{c}(x) \mid}}(b \cdot \nabla u)(X(\sigma ; x, \tilde{v}), \tilde{\mathcal{V}}(\sigma ; x, \tilde{v})) \mathrm{d} \sigma \mathrm{d} \theta
\end{aligned}
$$

As $u$ has compact support, its average $\langle u\rangle$ is also compactly supported and therefore $u-\langle u\rangle$ belongs to $L^{2}\left(\mathbb{R}^{2} \times \mathbb{R}^{2}\right)$. By the previous computation we have

$$
|\langle u\rangle(x, \tilde{v})-u(x, \tilde{v})| \leq \frac{1}{2 \pi} \int_{0}^{2 \pi} \int_{0}^{\frac{2 \pi}{\omega_{0}}}|(b \cdot \nabla u)(X(\sigma ; x, \tilde{v}), \tilde{\mathcal{V}}(\sigma ; x, \tilde{v}))| \mathrm{d} \sigma \mathrm{d} \theta
$$

and therefore

$$
\|\langle u\rangle-u\|_{L^{2}} \leq \frac{1}{2 \pi} \int_{0}^{2 \pi} \int_{0}^{\frac{2 \pi}{\omega_{0}}}\|(b \cdot \nabla u)(X(\sigma ; \cdot, \cdot), \tilde{\mathcal{V}}(\sigma ; \cdot, \cdot))\|_{L^{2}} \mathrm{~d} \sigma \mathrm{d} \theta=\frac{2 \pi}{\omega_{0}}\|b \cdot \nabla u\|_{L^{2}}
$$


The above Poincaré inequality still holds true for any $u \in L^{2}\left(\mathbb{R}^{2} \times \mathbb{R}^{2}\right)$ such that $b \cdot \nabla u \in L^{2}\left(\mathbb{R}^{2} \times \mathbb{R}^{2}\right)$, see [3] for details about the average of $L^{p}$ functions, $1 \leq p \leq \infty$.

6 . Observe that for any $(x, \tilde{v}) \in \mathbb{R}^{2} \times \mathbb{R}^{2}$ we have

$$
\langle u\rangle(x, \tilde{v})=\frac{1}{2 \pi} \int_{0}^{2 \pi} u(x, \mathcal{R}(-\theta) \tilde{v}) \mathrm{d} \theta
$$

and therefore $\langle u\rangle \in C^{1}\left(\mathbb{R}^{2} \times \mathbb{R}^{2}\right)$. Taking the derivatives with respect to $x, \tilde{v}$ under the integral sign, one gets

$$
\left(\nabla_{x}\langle u\rangle\right)(x, \tilde{v})=\frac{1}{2 \pi} \int_{0}^{2 \pi}\left(\nabla_{x} u\right)(x, \mathcal{R}(-\theta) \tilde{v}) \mathrm{d} \theta=\left\langle\nabla_{x} u\right\rangle(x, \tilde{v})
$$

and

$$
\tilde{v} \cdot\left(\nabla_{\tilde{v}}\langle u\rangle\right)(x, \tilde{v})=\frac{1}{2 \pi} \int_{0}^{2 \pi} \mathcal{R}(-\theta) \tilde{v} \cdot\left(\nabla_{\tilde{v}} u\right)(x, \mathcal{R}(-\theta) \tilde{v}) \mathrm{d} \theta=\left\langle\tilde{v} \cdot \nabla_{\tilde{v}} u\right\rangle(x, \tilde{v}) .
$$

By the definition, the average $\langle u\rangle$ is constant along the flow of $b \cdot \nabla_{x, \tilde{v}}$, and therefore belongs to the kernel of $b \cdot \nabla_{x, \tilde{v}}$, that is ${ }^{\perp} \tilde{v} \cdot \nabla_{\tilde{v}}\langle u\rangle=0$.

It is easily seen that the periods $S, S^{\varepsilon}$ are invariant along the flows of $b \cdot \nabla_{x, \tilde{v}}, b^{\varepsilon} \cdot \nabla_{x, \tilde{v}}$ as well as the averages $\langle u\rangle,\langle u\rangle_{\varepsilon}$. If $u$ is a $C^{1}$ function, we have by periodicity

$$
\left\langle b \cdot \nabla_{x, \tilde{v}} u\right\rangle(x, \tilde{v})=\frac{1}{S(x, \tilde{v})} \int_{0}^{S(x, \tilde{v})} \frac{\mathrm{d}}{\mathrm{d} s} u(X(s), \tilde{\mathcal{V}}(s)) \mathrm{d} s=0, \quad(x, \tilde{v}) \in \mathbb{R}^{2} \times \mathbb{R}^{2}
$$

and similarly $\left\langle b^{\varepsilon} \cdot \nabla_{x, \tilde{v}} u\right\rangle_{\varepsilon}=0$.

In the sequel we derive formally the limit model of (17), as $\varepsilon$ goes to 0 . The rigorous arguments will be discussed in Section 5. As we are interested by a second order model, we keep all the first order corrections. When $\varepsilon$ becomes small, we expect that $b^{\varepsilon}(x, \tilde{v}) \cdot \nabla_{x, \tilde{v}} \tilde{f}^{\varepsilon}$ will vanish, saying that $\tilde{f}^{\varepsilon}$ behaves like its average $\left\langle\tilde{f}^{\varepsilon}\right\rangle_{\varepsilon}$ along the flow $\left(X^{\varepsilon}, \tilde{\mathcal{V}}^{\varepsilon}\right)$. Taking the average of $(17)$ along the flow $\left(X^{\varepsilon}, \tilde{\mathcal{V}}^{\varepsilon}\right)$ we obtain

$$
\partial_{t}\left\langle\tilde{f}^{\varepsilon}(t)\right\rangle_{\varepsilon}+\varepsilon\left\langle a^{\varepsilon}\left[\tilde{f}^{\varepsilon}(t)\right] \cdot \nabla_{x, \tilde{v}} \tilde{f}^{\varepsilon}(t)\right\rangle_{\varepsilon}=0, \quad(t, x, \tilde{v}) \in \mathbb{R}_{+} \times \mathbb{R}^{2} \times \mathbb{R}^{2} .
$$

As the difference between the averages along $b^{\varepsilon}(x, \tilde{v}) \cdot \nabla_{x, \tilde{v}}$ and $b(x, \tilde{v}) \cdot \nabla_{x, \tilde{v}}$ is of order $\varepsilon$ cf. Proposition 3.1, up to a second order term, we can replace $\varepsilon\left\langle a^{\varepsilon}\left[\tilde{f}^{\varepsilon}(t)\right] \cdot \nabla_{x, \tilde{v}} \tilde{f}^{\varepsilon}(t)\right\rangle_{\varepsilon}$ by $\varepsilon\left\langle a[\tilde{f}(t)] \cdot \nabla_{x, \tilde{v}} \tilde{f}(t)\right\rangle$ where

$$
\begin{aligned}
a[\tilde{f}] \cdot \nabla_{x, \tilde{v}} & =\left(\frac{{ }^{\perp} E[\tilde{f}]}{B}-A_{x}\right) \cdot \nabla_{x} \\
& +\left[\frac{1}{2 \pi \varepsilon_{0} B} \operatorname{div}_{x} \int_{\mathbb{R}^{2}} \frac{{ }^{\perp}\left(x-x^{\prime}\right)}{\left|x-x^{\prime}\right|^{2}} \otimes j[\tilde{f}]\left(x^{\prime}\right) \mathrm{d} x^{\prime}-\partial_{x}\left(\frac{{ }^{\perp} E[\tilde{f}]}{B}\right) \tilde{v}\right] \cdot \nabla_{\tilde{v}}
\end{aligned}
$$

and $A_{x}(x, \tilde{v})=\frac{{ }^{\perp} \tilde{v} \otimes \tilde{v}}{\omega_{c}^{2}(x)} \nabla \omega_{c}$. More exactly, we expect that solving

$$
\partial_{t} \tilde{f}+\varepsilon\left\langle a[\tilde{f}(t)] \cdot \nabla_{x, \tilde{v}} \tilde{f}(t)\right\rangle=0, \quad b \cdot \nabla_{x, \tilde{v}} \tilde{f}=0, \quad(t, x, \tilde{v}) \in \mathbb{R}_{+} \times \mathbb{R}^{2} \times \mathbb{R}^{2}
$$


for a suitable (well prepared) initial condition (see Section 5), will provide a second order approximation for (1), (2). Although the above solution depends on $\varepsilon$, we use the notation $\tilde{f}$, for saying that it is an approximation of $\tilde{f}^{\varepsilon}$, when $\varepsilon$ becomes small. It remains to compute the average of $a[\tilde{f}(t)] \cdot \nabla_{x, \tilde{v}} \tilde{f}(t)$, where the particle density $\tilde{f}(t)$ satisfies the constraint $b \cdot \nabla_{x, \tilde{v}} \tilde{f}(t)=0$, that is, $\tilde{f}(t)$ depends only on $x$ and $|\tilde{v}|$. By the definition of the average operator along $b \cdot \nabla_{x, \tilde{v}}$, it is easily seen that $\left\langle{ }^{\perp} \tilde{v} \otimes \tilde{v}\right\rangle=\frac{|\tilde{v}|^{2}}{2} \mathcal{R}(-\pi / 2)$ and therefore

$$
\begin{aligned}
\varepsilon\left\langle\left(\frac{{ }^{\perp} E[\tilde{f}]}{B}-A_{x}\right) \cdot \nabla_{x} \tilde{f}\right\rangle & =\varepsilon \frac{{ }^{\perp} E[\tilde{f}]}{B(x)} \cdot \nabla_{x} \tilde{f}-\varepsilon\left\langle A_{x}\right\rangle \cdot \nabla_{x} \tilde{f} \\
& =\varepsilon\left(\frac{{ }^{\perp} E[\tilde{f}]}{B(x)}-{\frac{|\tilde{v}|^{2}}{2 \omega_{c}^{2}(x)}}^{\perp} \nabla \omega_{c}\right) \cdot \nabla_{x} \tilde{f}
\end{aligned}
$$

We recognize here the electric cross field drift $\varepsilon \frac{{ }^{\perp} E[\tilde{f}]}{B}=\frac{{ }^{\perp} E[\tilde{f}]}{B^{\varepsilon}}$ and the magnetic gradient drift

$$
-\varepsilon \frac{|\tilde{v}|^{2}}{2 \omega_{c}^{2}(x)}{ }^{\perp} \nabla \omega_{c}=-\frac{m|\tilde{v}|^{2}}{2 q B^{\varepsilon}} \frac{\perp \nabla B^{\varepsilon}}{B^{\varepsilon}} .
$$

$\operatorname{As}\left\langle\nabla_{\tilde{v}} \tilde{f}\right\rangle=0$, we have

$$
\left\langle\frac{1}{2 \pi \varepsilon_{0} B} \operatorname{div}_{x} \int_{\mathbb{R}^{2}} \frac{\perp\left(x-x^{\prime}\right)}{\left|x-x^{\prime}\right|^{2}} \otimes j[\tilde{f}]\left(x^{\prime}\right) \mathrm{d} x^{\prime} \cdot \nabla_{\tilde{v}} \tilde{f}\right\rangle=0 .
$$

It remains to compute the average $-\varepsilon\left\langle\partial_{x}\left(\frac{{ }^{\perp} E[\tilde{f}]}{B}\right) \tilde{v} \cdot \nabla_{\tilde{v}} \tilde{f}\right\rangle$. As $\tilde{f}$ satisfies the constraint $b \cdot \nabla_{x, \tilde{v}} \tilde{f}=0$, we have $\nabla_{\tilde{v}} \tilde{f}=\frac{\nabla_{\tilde{\tau}} \tilde{f} \cdot \tilde{v}}{|\tilde{v}|^{2}} \tilde{v}$ and therefore

$$
\begin{aligned}
-\varepsilon\left\langle\partial_{x}\left(\frac{{ }^{\perp} E[\tilde{f}]}{B}\right) \tilde{v} \cdot \nabla_{\tilde{v}} \tilde{f}\right\rangle & =\varepsilon\left\langle\partial_{x}\left(\frac{E[\tilde{f}]}{B}\right) \tilde{v} \cdot{ }^{\perp} \tilde{v}\right\rangle \frac{\nabla_{\tilde{v}} \tilde{f} \cdot \tilde{v}}{|\tilde{v}|^{2}} \\
& =\varepsilon \partial_{x}\left(\frac{E[\tilde{f}]}{B}\right):\left\langle{ }^{\perp} \tilde{v} \otimes \tilde{v}\right\rangle \frac{\nabla_{\tilde{v}} \tilde{f} \cdot \tilde{v}}{|\tilde{v}|^{2}} \\
& =\frac{\varepsilon}{2}\left(\frac{{ }^{\perp} E[\tilde{f}]}{B} \cdot \frac{\nabla B}{B}\right) \tilde{v} \cdot \nabla_{\tilde{v}} \tilde{f}
\end{aligned}
$$

Finally, the particle density $\tilde{f}$ satisfies

$$
\partial_{t} \tilde{f}+\left(\frac{{ }^{\perp} E[\tilde{f}]}{B^{\varepsilon}(x)}-\frac{m|\tilde{v}|^{2}}{2 q B^{\varepsilon}} \frac{{ }^{\perp} \nabla B^{\varepsilon}}{B^{\varepsilon}(x)}\right) \cdot \nabla_{x} \tilde{f}+\frac{1}{2}\left(\frac{{ }^{\perp} E[\tilde{f}]}{B^{\varepsilon}(x)} \cdot \frac{\nabla B^{\varepsilon}}{B^{\varepsilon}(x)}\right) \tilde{v} \cdot \nabla_{\tilde{v}} \tilde{f}=0 .
$$

\section{Remark 3.2}

The above model can also be obtained by working at the characteristic level. Indeed, at least formally, when $\varepsilon$ is small, the solution of (19) is approximated by the solution $\tilde{g}$ (which depends on $\varepsilon$ ) of

$$
\partial_{t} \tilde{g}+\varepsilon a[\tilde{g}(t)] \cdot \nabla_{x, \tilde{v}} \tilde{g}+\frac{b(x, \tilde{v})}{\varepsilon} \cdot \nabla_{x, \tilde{v}} \tilde{g}=0, \quad(t, x, \tilde{v}) \in \mathbb{R}_{+} \times \mathbb{R}^{2} \times \mathbb{R}^{2}
$$


whose characteristics, denoted by $(X, \tilde{V})$ (and depending on $\varepsilon$ ), verify

$$
\begin{gathered}
\frac{\mathrm{d} X}{\mathrm{~d} t}=\varepsilon a_{x}[\tilde{g}(t)](X(t), \tilde{V}(t)) \\
\frac{\mathrm{d} \tilde{V}}{\mathrm{~d} t}=\varepsilon a_{\tilde{v}}[\tilde{g}(t)](X(t), \tilde{V}(t))+\frac{\omega_{c}(X(t))}{\varepsilon}{ }^{\perp} \tilde{V}(t) .
\end{gathered}
$$

Studying the asymptotic behavior, when $\varepsilon$ becomes small, of (19) or (21), reduces to determining the effective characteristic system coming from (22), (23), when averaging with respect to the fast rotation in velocity. More exactly, (23) writes

$$
\frac{\mathrm{d}}{\mathrm{d} t}\left\{\mathcal{R}\left(\frac{\int_{0}^{t} \omega_{c}(X(s)) \mathrm{d} s}{\varepsilon}\right) \tilde{V}(t)\right\}=\varepsilon \mathcal{R}\left(\frac{\int_{0}^{t} \omega_{c}(X(s)) \mathrm{d} s}{\varepsilon}\right) a_{\tilde{v}}[\tilde{g}(t)](X(t), \tilde{V}(t))
$$

saying that $V(t):=\mathcal{R}\left(\frac{\int_{0}^{t} \omega_{c}(X(s)) \mathrm{d} s}{\varepsilon}\right) \tilde{V}(t)$ has no fast oscillations. Moreover, when $\varepsilon$ is small, the time variation of $V(t)$ can be approximated by

$$
\begin{aligned}
\frac{\varepsilon}{2 \pi} & \int_{0}^{2 \pi} \mathcal{R}(\theta) a_{\tilde{v}}[\tilde{g}(t)](X(t), \mathcal{R}(-\theta) V(t)) \mathrm{d} \theta \\
& =-\frac{\varepsilon}{2 \pi} \int_{0}^{2 \pi} \mathcal{R}(\theta) \partial_{x}\left(\frac{{ }^{\perp} E[\tilde{g}]}{B}\right)(X(t)) \mathcal{R}(-\theta) V(t) \mathrm{d} \theta \\
& =-\frac{\varepsilon}{2} \operatorname{div}_{x}\left(\frac{{ }^{\perp} E[\tilde{g}]}{B}\right)(X(t)) V(t)-\frac{\varepsilon}{2} \operatorname{div}_{x}\left(\frac{E[\tilde{g}]}{B}\right)(X(t))^{\perp} V(t) .
\end{aligned}
$$

In the above computation we have used the formula

$$
\frac{1}{2 \pi} \int_{0}^{2 \pi} \mathcal{R}(\theta) M \mathcal{R}(-\theta) \mathrm{d} \theta=\frac{\operatorname{trace}(M)}{2} I_{2}+\frac{\operatorname{trace}\left(\mathcal{R}\left(\frac{\pi}{2}\right) M\right)}{2} \mathcal{R}\left(-\frac{\pi}{2}\right)
$$

for any square matrix $M$ of size 2 . As the limit density $\tilde{g}$ satisfies the constraint $\omega_{c}(x)^{\perp} \tilde{v} \cdot \nabla_{\tilde{v}} \tilde{g}=0$, the velocity advection in the effective Vlasov equation is

$$
-\frac{\varepsilon}{2} \operatorname{div}_{x}\left(\frac{{ }^{\perp} E[\tilde{g}]}{B}\right) \tilde{v} \cdot \nabla_{\tilde{v}} \tilde{g}=\frac{1}{2}\left(\frac{{ }^{\perp} E[\tilde{g}]}{B^{\varepsilon}(x)} \cdot \frac{\nabla B^{\varepsilon}}{B^{\varepsilon}(x)}\right) \tilde{v} \cdot \nabla_{\tilde{v}} \tilde{g} .
$$

Notice that the acceleration $\frac{1}{2}\left(\frac{{ }^{\perp} E}{B^{\varepsilon}(x)} \cdot \frac{\nabla B^{\varepsilon}}{B^{\varepsilon}(x)}\right) \tilde{v}$ comes by smoothing out the fast velocity rotation, when computing the average

$$
-\frac{\varepsilon}{2 \pi} \int_{0}^{2 \pi} \mathcal{R}(\theta) \partial_{x}\left(\frac{{ }^{\perp} E}{B}\right)(X(t)) \mathcal{R}(-\theta) \mathrm{d} \theta V(t) .
$$

Similarly, the time variation of $X(t)$ can be approximated by

$$
\frac{\varepsilon}{2 \pi} \int_{0}^{2 \pi} a_{x}[\tilde{g}(t)](X(t), \mathcal{R}(-\theta) V(t)) \mathrm{d} \theta
$$


and therefore, by using one more time (24), one gets

$$
\begin{aligned}
& \frac{\varepsilon}{2 \pi} \int_{0}^{2 \pi}\left[\left(\frac{{ }^{\perp} E[\tilde{g}]}{B}\right)(X(t))-\frac{\mathcal{R}(-\theta){ }^{\perp} V(t) \otimes \mathcal{R}(-\theta) V(t)}{\omega_{c}^{2}(X(t))} \nabla \omega_{c}(X(t))\right] \mathrm{d} \theta \\
& \quad=\varepsilon\left(\frac{{ }^{\perp} E[\tilde{g}]}{B}\right)(X(t))-\frac{\varepsilon}{2 \pi} \int_{0}^{2 \pi} \mathcal{R}(-\theta) \frac{{ }^{\perp} V(t) \otimes V(t)}{\omega_{c}^{2}(X(t))} \mathcal{R}(\theta) \mathrm{d} \theta \nabla \omega_{c}(X(t)) \\
&= \varepsilon\left(\frac{{ }^{\perp} E[\tilde{g}]}{B}\right)(X(t))-\frac{\varepsilon}{2} \operatorname{trace}\left(\frac{{ }^{\perp} V(t) \otimes V(t)}{\omega_{c}^{2}(X(t))}\right) \nabla \omega_{c}(X(t)) \\
&-\frac{\varepsilon}{2} \operatorname{trace}\left(\mathcal{R}\left(\frac{\pi}{2}\right) \frac{{ }^{\perp} V(t) \otimes V(t)}{\omega_{c}^{2}(X(t))}\right){ }^{\perp} \nabla \omega_{c}(X(t)) \\
&=\varepsilon\left(\frac{{ }^{\perp} E[\tilde{g}]}{B}\right)(X(t))-\varepsilon \frac{|V(t)|^{2}}{2 \omega_{c}^{2}(X(t))}{ }^{\perp} \nabla \omega_{c}(X(t)) .
\end{aligned}
$$

The space advection in the effective Vlasov equation is given by the electric cross field drift and the magnetic gradient drift

$$
\left(\frac{{ }^{\perp} E[\tilde{g}]}{B^{\varepsilon}(x)}-\frac{m|\tilde{v}|^{2}}{2 q B^{\varepsilon}(x)} \frac{{ }^{\perp} \nabla B^{\varepsilon}}{B^{\varepsilon}(x)}\right) \cdot \nabla_{x} \tilde{g}
$$

and therefore we retrieve (20). Observe that the drift velocities come by smoothing out the fast velocity rotation, when computing the average

$$
\frac{\varepsilon}{2 \pi} \int_{0}^{2 \pi}\left[\left(\frac{{ }^{\perp} E}{B}\right)(X(t))-\frac{\mathcal{R}(-\theta){ }^{\perp} V(t) \otimes \mathcal{R}(-\theta) V(t)}{\omega_{c}^{2}(X(t))} \nabla \omega_{c}(X(t))\right] \mathrm{d} \theta .
$$

\section{Well posedness of the limit model}

In this section we focus on the existence, uniqueness and other properties of the limit model

$$
\partial_{t} \tilde{f}+\left(\frac{{ }^{\perp} E[\tilde{f}]}{B^{\varepsilon}(x)}-\frac{m|\tilde{v}|^{2}}{2 q B^{\varepsilon}} \frac{{ }^{\perp} \nabla B^{\varepsilon}}{B^{\varepsilon}(x)}\right) \cdot \nabla_{x} \tilde{f}+\frac{1}{2}\left(\frac{{ }^{\perp} E[\tilde{f}]}{B^{\varepsilon}(x)} \cdot \frac{\nabla B^{\varepsilon}}{B^{\varepsilon}(x)}\right) \tilde{v} \cdot \nabla_{\tilde{v}} \tilde{f}=0
$$

where $E[\tilde{f}]$ stands for the Poisson electric field

$$
E[\tilde{f}(t)](x)=\frac{q}{2 \pi \varepsilon_{0}} \int_{\mathbb{R}^{2}} \int_{\mathbb{R}^{2}} \tilde{f}\left(t, x^{\prime}, v^{\prime}\right) \frac{x-x^{\prime}}{\left|x-x^{\prime}\right|^{2}} \mathrm{~d} v^{\prime} \mathrm{d} x^{\prime}, \quad(t, x) \in \mathbb{R}_{+} \times \mathbb{R}^{2} .
$$

Although the well posedness of this limit model is analogous to that of the VlasovPoisson system, it is instructive to detail it there. A very important consequence of the uniqueness is the invariance of the solution under the rotations in the velocity space, cf. 6. Remark 4.1. In particular we justify the propagation of the constraint $b \cdot \nabla_{x, \tilde{v}} \tilde{f}=0$. We supplement our model by the initial condition

$$
\tilde{f}(0, x, \tilde{v})=\tilde{f}_{\text {in }}(x, \tilde{v}), \quad(x, \tilde{v}) \in \mathbb{R}^{2} \times \mathbb{R}^{2}
$$

where $\tilde{f}_{\text {in }}$ is a smooth compactly supported particle density. The parameter $\varepsilon>0$ is fixed and $B^{\varepsilon}$ is a smooth external magnetic field. 
Proof. (of Theorem 1.1)

We follow the same arguments as in the well posedness proof for the Vlasov-Poisson problem with external magnetic field. It is enough to establish the existence and uniqueness on any time interval $[0, T], T \in \mathbb{R}_{+}$. We construct a map acting on electric fields. Given a $C^{1}$ electric field $E$ on $[0, T] \times \mathbb{R}^{2}$ such that $E, \partial_{x} E$ are bounded, $\operatorname{div}_{x}{ }^{\perp} E=0$, we consider the solution by characteristics of the Vlasov problem (25), (27) on $[0, T] \times \mathbb{R}^{2} \times \mathbb{R}^{2}$, corresponding to the electric field $E$

$$
\tilde{f}^{E}(t, x, \tilde{v})=\tilde{f}_{\text {in }}\left(X^{E}(0 ; t, x, \tilde{v}), \tilde{V}^{E}(0 ; t, x, \tilde{v})\right), \quad(t, x, \tilde{v}) \in[0, T] \times \mathbb{R}^{2} \times \mathbb{R}^{2}
$$

where this time the characteristics $\left(X^{E}(s ; t, x, \tilde{v}), V^{E}(s ; t, x, \tilde{v})\right)$ are given by

$$
\begin{gathered}
\frac{\mathrm{d} X^{E}}{\mathrm{~d} s}=\frac{{ }^{\perp} E\left(s, X^{E}(s)\right)}{B^{\varepsilon}\left(X^{E}(s)\right)}-\frac{m\left|\tilde{V}^{E}(s)\right|^{2}}{2 q B^{\varepsilon}\left(X^{E}(s)\right)} \frac{{ }^{\perp} \nabla B^{\varepsilon}\left(X^{E}(s)\right)}{B^{\varepsilon}\left(X^{E}(s)\right)} \\
\frac{\mathrm{d} \tilde{V}^{E}}{\mathrm{~d} s}=\frac{1}{2}\left(\frac{{ }^{\perp} E\left(s, X^{E}(s)\right)}{B^{\varepsilon}\left(X^{E}(s)\right)} \cdot \frac{\nabla B^{\varepsilon}\left(X^{E}(s)\right)}{B^{\varepsilon}\left(X^{E}(s)\right)}\right) \tilde{V}^{E}(s) \\
X^{E}(s ; t, x, \tilde{v})=x, \quad \tilde{V}^{E}(s ; t, x, \tilde{v})=\tilde{v}, \quad(s, t, x, \tilde{v}) \in[0, T] \times[0, T] \times \mathbb{R}^{2} \times \mathbb{R}^{2} .
\end{gathered}
$$

Notice that the magnetic moment $\mu^{\varepsilon}(x, \tilde{v})=\frac{m|\tilde{v}|^{2}}{2 B^{\varepsilon}(x)}$ is left invariant along these characteristics :

$$
\begin{aligned}
\frac{\mathrm{d} \mu^{\varepsilon}}{\mathrm{d} s} & =-\frac{m\left|\tilde{V}^{E}(s)\right|^{2}}{2 B^{\varepsilon}\left(X^{E}(s)\right)} \frac{\nabla B^{\varepsilon}\left(X^{E}(s)\right)}{B^{\varepsilon}\left(X^{E}(s)\right)} \cdot\left(\frac{{ }^{\perp} E\left(s, X^{E}(s)\right)}{B^{\varepsilon}\left(X^{E}(s)\right)}-\frac{m\left|\tilde{V}^{E}(s)\right|^{2}}{2 q B^{\varepsilon}\left(X^{E}(s)\right)} \frac{{ }^{\perp} \nabla B^{\varepsilon}\left(X^{E}(s)\right)}{B^{\varepsilon}\left(X^{E}(s)\right)}\right) \\
& +\frac{m\left|\tilde{V}^{E}(s)\right|^{2}}{2 B^{\varepsilon}\left(X^{E}(s)\right)}\left(\frac{{ }^{\perp} E\left(s, X^{E}(s)\right)}{B^{\varepsilon}\left(X^{E}(s)\right)} \cdot \frac{\nabla B^{\varepsilon}\left(X^{E}(s)\right)}{B^{\varepsilon}\left(X^{E}(s)\right)}\right)=0
\end{aligned}
$$

and therefore the velocity remains bounded, independently with respect to the electric field

$$
\left|\tilde{V}^{E}(s)\right| \leq\left(\frac{\left\|B^{\varepsilon}\right\|_{L^{\infty}}}{B_{0}^{\varepsilon}}\right)^{1 / 2}|\tilde{v}|=\left(\frac{\|B\|_{L^{\infty}}}{B_{0}}\right)^{1 / 2}|\tilde{v}|, \quad s \in \mathbb{R} .
$$

We deduce that (28) also writes

$$
\frac{\mathrm{d} X^{E}}{\mathrm{~d} s}=\frac{{ }^{\perp} E\left(s, X^{E}(s)\right)}{B^{\varepsilon}\left(X^{E}(s)\right)}-\frac{m|\tilde{v}|^{2}}{2 q B^{\varepsilon}(x)} \frac{{ }^{\perp} \nabla B^{\varepsilon}\left(X^{E}(s)\right)}{B^{\varepsilon}\left(X^{E}(s)\right)}
$$

whose right hand side remains bounded for $s \in[0, T]$ and thus $X^{E}$ exists globally on $[0, T]$ and has $C^{1}$ regularity in all the arguments. Once that $X^{E}$ is determined, $\tilde{V}^{E}$ comes easily by solving (29), which is linear with respect to $\tilde{V}^{E}$, and therefore $\tilde{V}^{E}$ exists also globally on $[0, T]$, and has $C^{1}$ regularity in all the arguments. As before, we consider the map $\tilde{\mathcal{F}}$, whose fixed point gives the solution of the limit model $(25),(26)$, (27)

$$
E \rightarrow \tilde{\mathcal{F}}(E):=\frac{q}{2 \pi \varepsilon_{0}} \int_{\mathbb{R}^{2}} \int_{\mathbb{R}^{2}} \tilde{f}^{E}\left(t, x^{\prime}, \tilde{v}^{\prime}\right) \frac{x-x^{\prime}}{\left|x-x^{\prime}\right|^{2}} \mathrm{~d} v^{\prime} \mathrm{d} x^{\prime}
$$

We are looking for a set $\tilde{X}_{T}$ of smooth electric fields, which is left invariant by the map $\tilde{\mathcal{F}}$ and for an estimate like

$$
\left.\left.\left\|\tilde{\mathcal{F}} E(t)-\tilde{\mathcal{F}} E^{\prime}(t)\right\|_{L^{\infty}} \leq \varepsilon \tilde{C}_{T} \int_{0}^{t}\left\|E(s)-E^{\prime}(s)\right\|_{L^{\infty}} \mathrm{d} s, \quad E, E^{\prime} \in \tilde{X}_{T}, t \in[0, T], \varepsilon \in\right] 0,1\right]
$$


for some constant $\tilde{C}_{T}$, not depending on $E, E^{\prime}$. Assume that

$$
\operatorname{supp} \tilde{f}_{\text {in }} \subset\left\{(x, \tilde{v}) \in \mathbb{R}^{2} \times \mathbb{R}^{2}:|x| \leq \tilde{R}_{x}^{\text {in }} \text { and }|\tilde{v}| \leq \tilde{R}_{\tilde{v}}^{\text {in }}\right\} .
$$

By the conservation of the magnetic moment and of the particle number (thanks to the condition $\operatorname{div}_{x}{ }^{\perp} E=0$ ), it is easily seen that at any time $t \in[0, T]$ we have

$$
\operatorname{supp} \tilde{f}^{E}(t) \subset\left\{(x, \tilde{v}) \in \mathbb{R}^{2} \times \mathbb{R}^{2}:|\tilde{v}| \leq \tilde{R}_{\tilde{v}}:=\tilde{R}_{\tilde{v}}^{\text {in }}\left(\|B\|_{L^{\infty}} / B_{0}\right)^{1 / 2}\right\}
$$

implying that

$$
\|\rho[\tilde{f}(t)]\|_{L^{\infty}} \leq|q|\left\|\tilde{f}_{\text {in }}\right\|_{L^{\infty}} \pi\left(\tilde{R}_{\tilde{v}}^{\text {in }}\right)^{2} \frac{\|B\|_{L^{\infty}}}{B_{0}} .
$$

Therefore, as in (49) we obtain the $L^{\infty}$ bound for the electric field

$$
\begin{aligned}
\|E[\tilde{f}(t)]\|_{L^{\infty}\left(\mathbb{R}^{2}\right)} & \leq \frac{1}{\varepsilon_{0}}\|\rho[\tilde{f}(t)]\|_{L^{\infty}\left(\mathbb{R}^{2}\right)}^{1 / 2}\left(\frac{|q|}{2 \pi}\left\|\tilde{f}_{\text {in }}\right\|_{L^{1}\left(\mathbb{R}^{2} \times \mathbb{R}^{2}\right)}\right)^{1 / 2} \\
& \leq \alpha:=\frac{|q|}{\sqrt{2} \varepsilon_{0}}\left\|\tilde{f}_{\text {in }}\right\|_{L^{\infty}\left(\mathbb{R}^{2} \times \mathbb{R}^{2}\right)}^{1 / 2} R_{\tilde{v}}^{\text {in }}\left(\frac{\|B\|_{L^{\infty}}}{B_{0}}\right)^{1 / 2}\left\|\tilde{f}_{\text {in }}\right\|_{L^{1}\left(\mathbb{R}^{2} \times \mathbb{R}^{2}\right)}^{1 / 2}, \quad t \in[0, T] .
\end{aligned}
$$

Integrating in time (30) leads immediately to

$$
\operatorname{supp} \tilde{f}(t) \subset\left\{(x, \tilde{v}) \in \mathbb{R}^{2} \times \mathbb{R}^{2}:|x| \leq \tilde{R}_{x}(t) \text { and }|\tilde{v}| \leq \tilde{R}_{\tilde{v}}\right\}
$$

where

$$
\tilde{R}_{x}(t)=\tilde{R}_{x}^{\text {in }}+\varepsilon t\left[\frac{\alpha}{B_{0}}+\frac{m\left(\tilde{R}_{\tilde{v}}^{\text {in }}\right)^{2}}{2|q| B_{0}} \frac{\|B\|_{L^{\infty}}}{B_{0}} \frac{\|\nabla B\|_{L^{\infty}}}{B_{0}}\right], \quad t \in[0, T] .
$$

Taking the derivative with respect to $x$ in (30), (29) we deduce that there is a constant $C_{5}\left(m, \varepsilon_{0}, q, T,\|B\|_{W^{2, \infty}\left(\mathbb{R}^{2}\right)}, B_{0}\right)$ such that for any $(x, \tilde{v}) \in \mathbb{R}^{2} \times \mathbb{R}^{2},|\tilde{v}| \leq \tilde{R}_{\tilde{v}}$

$$
\left|\partial_{x} X^{E}(0 ; t, x, \tilde{v})\right|+\left|\partial_{x} \tilde{V}^{E}(0 ; t, x, \tilde{v})\right| \leq C_{5} \exp \left(\varepsilon \int_{0}^{t}\left\|\partial_{x} E(s)\right\|_{L^{\infty}} \mathrm{d} s\right), \quad t \in[0, T] .
$$

As before we write

$$
\begin{aligned}
\nabla_{x} \rho\left[\tilde{f}^{E}(t)\right] & =q \nabla_{x} \int_{\mathbb{R}^{2}} \tilde{f}_{\mathrm{in}}\left(X^{E}(0 ; t, x, \tilde{v}), \tilde{V}^{E}(0 ; t, x, \tilde{v})\right) \mathrm{d} \tilde{v} \\
& =q \int_{\mathbb{R}^{2}} \mathbf{1}_{\left\{|\tilde{v}| \leq \tilde{R}_{\tilde{v}}\right\}}{ }^{t} \partial_{x} X^{E}(0 ; t, x, \tilde{v})\left(\nabla_{X} \tilde{f}_{\mathrm{in}}\right)\left(X^{E}(0 ; t, x, \tilde{v}), \tilde{V}^{E}(0 ; t, x, \tilde{v})\right) \mathrm{d} \tilde{v} \\
& +q \int_{\mathbb{R}^{2}} \mathbf{1}_{\left\{|\tilde{v}| \leq \tilde{R}_{\tilde{v}\}}\right.}{ }^{t} \partial_{x} \tilde{V}^{E}(0 ; t, x, \tilde{v})\left(\nabla_{V} \tilde{f}_{\text {in }}\right)\left(X^{E}(0 ; t, x, \tilde{v}), \tilde{V}^{E}(0 ; t, x, \tilde{v})\right) \mathrm{d} \tilde{v}
\end{aligned}
$$

and by coming back to (32), we obtain

$$
\left\|\nabla_{x} \rho\left[\tilde{f}^{E}(t)\right]\right\|_{L^{\infty}} \leq C_{6} \exp \left(\varepsilon \int_{0}^{t}\left\|\partial_{x} E(s)\right\|_{L^{\infty}} \mathrm{d} s\right), \quad t \in[0, T]
$$

for some constant $C_{6}\left(m, \varepsilon_{0}, q, T,\|B\|_{W^{2, \infty}\left(\mathbb{R}^{2}\right)}, B_{0}\right) \geq 1$ and thus

$$
\left.\left.\ln ^{+}\left\|\nabla_{x} \rho\left[\tilde{f}^{E}(t)\right]\right\|_{L^{\infty}} \leq \ln C_{6}+\varepsilon \int_{0}^{t}\left\|\partial_{x} E(s)\right\|_{L^{\infty}} \mathrm{d} s, \quad t \in[0, T], \varepsilon \in\right] 0,1\right] .
$$


Combining with (54) yields

$$
\left.\left.\left\|\partial_{x} E\left[\tilde{f}^{E}(t)\right]\right\|_{L^{\infty}} \leq C_{7}\left(1+\varepsilon \int_{0}^{t}\left\|\partial_{x} E(s)\right\|_{L^{\infty}} \mathrm{d} s\right), \quad t \in[0, T], \varepsilon \in\right] 0,1\right]
$$

for some constant $C_{7}\left(m, \varepsilon_{0}, q, T,\|B\|_{W^{2, \infty}\left(\mathbb{R}^{2}\right)}, B_{0}\right)$. The previous computations allow us to consider the set

$$
\begin{aligned}
& \tilde{X}_{T}=\left\{E \in C^{1}\left([0, T] \times \mathbb{R}^{2}\right):\right. \\
& \left.\quad \operatorname{div}_{x}{ }^{\perp} E=0,\|E(t)\|_{L^{\infty}} \leq \alpha,\left\|\partial_{x} E(t)\right\|_{L^{\infty}} \leq C_{7} \exp \left(\varepsilon t C_{7}\right), t \in[0, T]\right\} .
\end{aligned}
$$

It remains to establish (31). Let us consider $E, E^{\prime} \in \tilde{X}_{T}$ and denote by $\tilde{f}{ }^{E}, \tilde{f}^{E^{\prime}}$ the solutions by characteristics of $(25),(27)$ corresponding to the electric fields $E, E^{\prime}$ respectively. Recall that at any time $t \in[0, T]$, the densities $\tilde{f}^{E}(t), \tilde{f}^{E^{\prime}}(t)$ are supported in $\left\{(x, \tilde{v}) \in \mathbb{R}^{2} \times \mathbb{R}^{2}:|x| \leq \tilde{R}_{x}(t)\right.$ and $\left.|\tilde{v}| \leq \tilde{R}_{\tilde{v}}\right\}$ and therefore at any time $t \in[0, T]$, the charge densities $\rho\left[\tilde{f}^{E}(t)\right], \rho\left[\tilde{f}^{E^{\prime}}(t)\right]$ are supported in $\left\{x \in \mathbb{R}^{2}:|x| \leq \tilde{R}_{x}(t)\right\}$. It is easily seen by (49) that

$$
\begin{aligned}
\| \tilde{\mathcal{F}}(E)(t) & -\tilde{\mathcal{F}}\left(E^{\prime}\right)(t)\left\|_{L^{\infty}\left(\mathbb{R}^{2}\right)}=\right\| E\left[\tilde{f}^{E}(t)\right]-E\left[\tilde{f}^{E^{\prime}}(t)\right] \|_{L^{\infty}\left(\mathbb{R}^{2}\right)} \\
& \leq \frac{1}{\varepsilon_{0}}\left\|\rho\left[\tilde{f}^{E}(t)\right]-\rho\left[\tilde{f}^{E^{\prime}}(t)\right]\right\|_{L^{\infty}\left(\mathbb{R}^{2}\right)}^{1 / 2}\left(\frac{1}{2 \pi}\left\|\rho\left[\tilde{f}^{E}(t)\right]-\rho\left[\tilde{f}^{E^{\prime}}(t)\right]\right\|_{L^{1}\left(\mathbb{R}^{2}\right)}\right)^{1 / 2} \\
& \leq \frac{1}{\varepsilon_{0}} \frac{|q|}{\sqrt{2}} \tilde{R}_{x}(t)\left\|\mathbf{1}_{\left\{|x| \leq \tilde{R}_{x}(t)\right\}} \int_{\mathbb{R}^{2}} \mathbf{1}_{\left\{|\tilde{v}| \leq \tilde{R}_{\tilde{v}}\right\}}\left(\tilde{f}^{E}-\tilde{f}^{E^{\prime}}\right)(t, \cdot \tilde{v}) \mathrm{d} \tilde{v}\right\|_{L^{\infty}\left(\mathbb{R}^{2}\right)} .
\end{aligned}
$$

By standard computations, one gets for any $(t, x, \tilde{v}) \in[0, T] \times \mathbb{R}^{2} \times \mathbb{R}^{2}$ such that $|x| \leq \tilde{R}_{x}(t)$ and $|\tilde{v}| \leq \tilde{R}_{\tilde{v}}$

$$
\begin{aligned}
\left|\left(\tilde{f}^{E}-\tilde{f}^{E^{\prime}}\right)(t, x, \tilde{v})\right| & =\left|\tilde{f}_{\text {in }}\left(X^{E}(0 ; t, x, \tilde{v}), \tilde{V}^{E}(0 ; t, x, \tilde{v})\right)-\tilde{f}_{\text {in }}\left(X^{E^{\prime}}(0 ; t, x, \tilde{v}), \tilde{V}^{E^{\prime}}(0 ; t, x, \tilde{v})\right)\right| \\
& \leq \operatorname{Lip}\left(f_{\text {in }}\right)\left[\left|X^{E}-X^{E^{\prime}}\right|(0 ; t, x, \tilde{v})+\left|\tilde{V}^{E}-\tilde{V}^{E^{\prime}}\right|(0 ; t, x, \tilde{v})\right] \\
& \leq \varepsilon \operatorname{Lip}\left(f_{\text {in }}\right) C_{8} \int_{0}^{t}\left\|E(s)-E^{\prime}(s)\right\|_{L^{\infty}\left(\mathbb{R}^{2}\right)} \mathrm{d} s \exp \left(\varepsilon t C_{8}\right)
\end{aligned}
$$

for some constant $C_{8}\left(m, \varepsilon_{0}, q, T,\|B\|_{W^{2, \infty}\left(\mathbb{R}^{2}\right)}, B_{0}\right)$ and (31) follows.

\section{Remark 4.1}

Under the hypotheses of Theorem 1.1, let us consider the solution $(\tilde{f}, E=E[\tilde{f}])$ of (25), (26). Therefore

1. The total number of particles is conserved. Indeed, as $\operatorname{div}_{x}^{\perp} E=0$, we have

$$
\operatorname{div}_{x}\left(\frac{{ }^{\perp} E}{B^{\varepsilon}}-\frac{m|\tilde{v}|^{2}}{2 q B^{\varepsilon}} \frac{{ }^{\perp} \nabla B^{\varepsilon}}{B^{\varepsilon}}\right)+\operatorname{div}_{\tilde{v}}\left[\frac{1}{2}\left(\frac{{ }^{\perp} E}{B^{\varepsilon}} \cdot \frac{\nabla B^{\varepsilon}}{B^{\varepsilon}}\right) \tilde{v}\right]=0
$$

and thus

$$
\frac{\mathrm{d}}{\mathrm{d} t} \int_{\mathbb{R}^{2}} \int_{\mathbb{R}^{2}} \tilde{f}(t, x, \tilde{v}) \mathrm{d} \tilde{v} \mathrm{~d} x=0, \quad t \in \mathbb{R}_{+}
$$


2. The continuity equation writes

$$
\partial_{t} \int_{\mathbb{R}^{2}} \tilde{f}(t, x, \tilde{v}) \mathrm{d} \tilde{v}+\operatorname{div}_{x} \int_{\mathbb{R}^{2}}\left(\frac{{ }^{\perp} E}{B^{\varepsilon}}-\frac{m|\tilde{v}|^{2}}{2 q B^{\varepsilon}} \frac{{ }^{\perp} \nabla B^{\varepsilon}}{B^{\varepsilon}}\right) \tilde{f} \mathrm{~d} \tilde{v}=0
$$

and, when the magnetic field is uniform, becomes

$$
\partial_{t} \rho[\tilde{f}(t)]+\frac{{ }^{\perp} E[\tilde{f}(t)]}{B^{\varepsilon}} \cdot \nabla_{x} \rho[\tilde{f}(t)]=0, \quad(t, x) \in \mathbb{R}_{+} \times \mathbb{R}^{2}
$$

that is, the vorticity formulation of the two dimensional Euler equations, with velocity ${ }^{\perp} E / B^{\varepsilon}$ and vorticity $\operatorname{div}_{x}{ }^{\perp}\left(\frac{{ }^{\perp} E}{B^{\varepsilon}}\right)=-\frac{\rho[\tilde{f}]}{\varepsilon_{0} B^{\varepsilon}}$ see also [12, 4, 27].

3. The balance of the total momentum is

$$
\frac{\mathrm{d}}{\mathrm{d} t} \int_{\mathbb{R}^{2}} \int_{\mathbb{R}^{2}} \tilde{f}(t, x, \tilde{v}) m \tilde{v} \mathrm{~d} \tilde{v} \mathrm{~d} x+\frac{1}{2} \int_{\mathbb{R}^{2}} \int_{\mathbb{R}^{2}} m \operatorname{div}_{x}\left(\frac{{ }^{\perp} E[\tilde{f}]}{B^{\varepsilon}(x)}\right) \tilde{f}(t, x, \tilde{v}) \tilde{v} \mathrm{~d} \tilde{v} \mathrm{~d} x=0 .
$$

In particular, the total momentum is conserved when the magnetic field is uniform.

4. As the magnetic moment $\mu^{\varepsilon}$ is left invariant along the characteristics of (25)

$$
\left(\frac{{ }^{\perp} E[\tilde{f}]}{B^{\varepsilon}}-\mu^{\varepsilon} \frac{{ }^{\perp} \nabla B^{\varepsilon}}{B^{\varepsilon}}\right) \cdot \nabla_{x} \mu^{\varepsilon}+\frac{1}{2}\left(\frac{{ }^{\perp} E[\tilde{f}]}{B^{\varepsilon}} \cdot \frac{\nabla B^{\varepsilon}}{B^{\varepsilon}}\right) \tilde{v} \cdot \nabla_{\tilde{v}} \mu^{\varepsilon}=0
$$

we deduce the conservation of the total magnetic moment

$$
\frac{\mathrm{d}}{\mathrm{d} t} \int_{\mathbb{R}^{2}} \int_{\mathbb{R}^{2}} \frac{m|\tilde{v}|^{2}}{2 B^{\varepsilon}} \tilde{f}(t, x, \tilde{v}) \mathrm{d} \tilde{v} \mathrm{~d} x=0, \quad t \in \mathbb{R}_{+} .
$$

5. The total, i.e., kinetic and electric energy is conserved. Indeed, by using the fundamental solution of the Poisson equation in $\mathbb{R}^{2}$, we have

$$
\begin{aligned}
\frac{\mathrm{d}}{\mathrm{d} t} \frac{1}{2 \varepsilon_{0}} & \int_{\mathbb{R}^{2}} \int_{\mathbb{R}^{2}} e\left(x-x^{\prime}\right) \rho[\tilde{f}(t)](x) \rho[\tilde{f}(t)]\left(x^{\prime}\right) \mathrm{d} x^{\prime} \mathrm{d} x \\
& =\frac{1}{\varepsilon_{0}} \int_{\mathbb{R}^{2}} \int_{\mathbb{R}^{2}} e\left(x-x^{\prime}\right) \rho[\tilde{f}(t)]\left(x^{\prime}\right) \partial_{t} \rho[\tilde{f}(t)](x) \mathrm{d} x^{\prime} \mathrm{d} x \\
& =\int_{\mathbb{R}^{2}} \Phi[\tilde{f}(t)](x) \partial_{t} \rho[\tilde{f}(t)](x) \mathrm{d} x \\
& =-\int_{\mathbb{R}^{2}} \Phi[\tilde{f}(t)](x) \operatorname{div}_{x} \int_{\mathbb{R}^{2}} q \tilde{f}(t, x, \tilde{v})\left(\frac{{ }^{\perp} E[\tilde{f}(t)]}{B^{\varepsilon}}-\frac{m|\tilde{v}|^{2} \perp}{2 q B^{\varepsilon}} \frac{{ }^{\varepsilon} B^{\varepsilon}}{B^{\varepsilon}}\right) \mathrm{d} \tilde{v} \mathrm{~d} x \\
& =\int_{\mathbb{R}^{2}} \int_{\mathbb{R}^{2}}\left(\frac{E[\tilde{f}(t)]}{B^{\varepsilon}} \cdot \frac{{ }^{\perp} \nabla B^{\varepsilon}}{B^{\varepsilon}}\right) \frac{m|\tilde{v}|^{2}}{2} \tilde{f} \mathrm{~d} \tilde{v} \mathrm{~d} x \\
& =-\frac{\mathrm{d}}{\mathrm{d} t} \int_{\mathbb{R}^{2}} \int_{\mathbb{R}^{2}} \frac{m|\tilde{v}|^{2}}{2} \tilde{f}(t, x, \tilde{v}) \mathrm{d} \tilde{v} \mathrm{~d} x
\end{aligned}
$$

and therefore

$\frac{\mathrm{d}}{\mathrm{d} t}\left[\int_{\mathbb{R}^{2}} \int_{\mathbb{R}^{2}} \frac{m|\tilde{v}|^{2}}{2} \tilde{f} \mathrm{~d} \tilde{v} \mathrm{~d} x-\frac{1}{4 \pi \varepsilon_{0}} \int_{\mathbb{R}^{2}} \int_{\mathbb{R}^{2}} \rho[\tilde{f}](x) \rho[\tilde{f}]\left(x^{\prime}\right) \ln \left|x-x^{\prime}\right| \mathrm{d} x^{\prime} \mathrm{d} x\right]=0, \quad t \in \mathbb{R}_{+}$. 
6. The model (25), (26), (27) is invariant under rotation in the velocity space. More exactly, if $\tilde{f}$ solves $(25),(26),(27)$, then $\tilde{f}_{\theta}(t, x, \tilde{V})=\tilde{f}(t, x, \tilde{v}=\mathcal{R}(\theta) \tilde{V})$ solves (25), (26) as well, together with the initial condition $\tilde{f}_{\theta}(0, x, \tilde{V})=\tilde{f}_{\text {in }}(x, \tilde{v}=$ $\mathcal{R}(\theta) \tilde{V})$. Indeed, we have $\tilde{V} \cdot \nabla_{\tilde{V}} \tilde{f}_{\theta}=\left.\tilde{v} \cdot \nabla_{\tilde{v}} \tilde{f}\right|_{\tilde{v}=\mathcal{R}(\theta) \tilde{V}}$ and $E\left[\tilde{f}_{\theta}(t)\right]=E[\tilde{f}(t)]$, implying that

$\partial_{t} \tilde{f}_{\theta}+\left(\frac{{ }^{\perp} E\left[\tilde{f}_{\theta}\right]}{B^{\varepsilon}}-\frac{m|\tilde{V}|^{2}}{2 q B^{\varepsilon}} \frac{{ }^{\perp} \nabla B^{\varepsilon}}{B^{\varepsilon}}\right) \cdot \nabla_{x} \tilde{f}_{\theta}+\frac{1}{2}\left(\frac{{ }^{\perp} E\left[\tilde{f}_{\theta}(t)\right]}{B^{\varepsilon}} \cdot \frac{\nabla B^{\varepsilon}}{B^{\varepsilon}}\right)\left(\tilde{V} \cdot \nabla_{\tilde{V}} \tilde{f}_{\theta}\right)=0$.

In particular, if the initial particle density depends only on $x$ and $|\tilde{v}|$ i.e., $b$. $\nabla_{x, \tilde{v}} \tilde{f}_{\text {in }}=0$, then at any time $t \in \mathbb{R}_{+}$the particle density $\tilde{f}(t)$ depends only on $x$ and $|\tilde{v}|$, that is $b \cdot \nabla_{x, \tilde{v}} \tilde{f}(t)=0$. Indeed, for any $\theta \in \mathbb{R}$ the particle densities $\tilde{f}, \tilde{f}_{\theta}$ satisfy the problem (25), (26), (27) and thanks to the uniqueness, we obtain $\tilde{f}=\tilde{f}_{\theta}$, saying that at any time $t \in \mathbb{R}_{+}, \tilde{f}(t)$ depends only on $x$ and $|\tilde{v}|$.

\section{$5 \quad$ Error analysis}

In many of the previous works concerning the asymptotic regimes for the magnetic confinement, the authors indicate convergence results, based on compactness arguments. We intend to perform here the error analysis. In this section we study rigorously the error when replacing the Vlasov-Poisson problem with strong external magnetic field (1), (2) by the model (25), (26). We expect an error of order $\varepsilon^{2}$, when the initial particle density $f_{\text {in }}$ is well prepared. The case with general initial particle densities is more elaborated, but can be handled by similar arguments cf. [9]. As usual when performing the error analysis in homogenization theory, we need to construct a corrector. We will use the following easy lemma, see also (24).

Lemma 5.1 Let $M$ be a square matrix of size 2 . Then we have the equalities

$$
\begin{gathered}
\langle M \tilde{v} \cdot \tilde{v}\rangle=\operatorname{trace}(M) \frac{|\tilde{v}|^{2}}{2} \\
M \tilde{v} \cdot \tilde{v}-\langle M \tilde{v} \cdot \tilde{v}\rangle+b \cdot \nabla_{x, \tilde{v}}\left(\frac{M \mathcal{R}(-\pi / 2)+\mathcal{R}(\pi / 2) M}{4 \omega_{c}(x)} \tilde{v} \cdot \tilde{v}\right)=0 \\
\left\langle\frac{M \mathcal{R}(-\pi / 2)+\mathcal{R}(\pi / 2) M}{4 \omega_{c}(x)} \tilde{v} \cdot \tilde{v}\right\rangle=0 .
\end{gathered}
$$

Proof. By direct computations we check that $\left\langle\tilde{v}_{1}^{2}\right\rangle=\left\langle\tilde{v}_{2}^{2}\right\rangle=|\tilde{v}|^{2} / 2,\left\langle\tilde{v}_{1} \tilde{v}_{2}\right\rangle=0$,

$$
b \cdot \nabla_{x, \tilde{v}}\left(\tilde{v}_{1}^{2}-\tilde{v}_{2}^{2}\right)=4 \omega_{c}(x) \tilde{v}_{2} \tilde{v}_{1}, \quad b \cdot \nabla_{x, \tilde{v}}\left(\tilde{v}_{2} \tilde{v}_{1}\right)=\omega_{c}(x)\left(\tilde{v}_{2}^{2}-\tilde{v}_{1}^{2}\right)
$$

and the first and second statements follow easily. The last statement is a consequence of the first one

$$
\left\langle\frac{M \mathcal{R}(-\pi / 2)+\mathcal{R}(\pi / 2) M}{4 \omega_{c}(x)} \tilde{v} \cdot \tilde{v}\right\rangle=\operatorname{trace}\left(M \mathcal{R}\left(-\frac{\pi}{2}\right)+\mathcal{R}\left(\frac{\pi}{2}\right) M\right) \frac{|\tilde{v}|^{2}}{8 \omega_{c}(x)}=0
$$


Looking for second order estimates is a difficult task. Indeed, this can not be achieved directly, by comparing $\tilde{f}^{\varepsilon}$ and $\tilde{f}$, but requires the construction of a corrector $\tilde{f}^{2}$. Since the smoothness of the corrector is crucial when analyzing the error, we indicate below its exact expression.

Proposition 5.1 Assume that $\tilde{f}=\tilde{f}(x, \tilde{v}) \in C_{c}^{1}\left(\mathbb{R}^{2} \times \mathbb{R}^{2}\right), E=E(x) \in C^{1}\left(\mathbb{R}^{2}\right), B=$ $B(x) \in C^{1}\left(\mathbb{R}^{2}\right)$ such that $b \cdot \nabla_{x, \tilde{v}} \tilde{f}=0, \operatorname{div}_{x}{ }^{\perp} E=0, \inf _{x \in \mathbb{R}^{2}}|B(x)|>0$. Then we have the equality

$$
\begin{aligned}
& \left(\frac{{ }^{\perp} E}{B}-A_{x}\right) \cdot \nabla_{x} \tilde{f}+\left[\frac{1}{2 \pi \varepsilon_{0} B} \operatorname{div}_{x} \int_{\mathbb{R}^{2}} \frac{{ }^{\perp}\left(x-x^{\prime}\right)}{\left|x-x^{\prime}\right|^{2}} \otimes j[\tilde{f}]\left(x^{\prime}\right) \mathrm{d} x^{\prime}-\partial_{x}\left(\frac{{ }^{\perp} E}{B}\right) \tilde{v}\right] \cdot \nabla_{\tilde{v}} \tilde{f} \\
& -\left(\frac{{ }^{\perp} E}{B}-\frac{m|\tilde{v}|^{2}}{2 q B} \frac{{ }^{\perp} \nabla B}{B}\right) \cdot \nabla_{x} \tilde{f}-\frac{1}{2}\left(\frac{{ }^{\perp} E}{B} \cdot \frac{\nabla B}{B}\right) \tilde{v} \cdot \nabla_{\tilde{v}} \tilde{f}+b \cdot \nabla_{x, \tilde{v}} \tilde{f}^{2}=0
\end{aligned}
$$

where $A_{x}(x, \tilde{v})=\frac{{ }^{\perp} \tilde{v} \otimes \tilde{v}}{\omega_{c}^{2}} \nabla \omega_{c}$ and

$$
\begin{aligned}
\tilde{f}^{2}(x, \tilde{v}) & =\frac{\nabla \omega_{c} \otimes \nabla_{x} \tilde{f}-{ }^{\perp} \nabla \omega_{c} \otimes{ }^{\perp} \nabla_{x} \tilde{f}}{4 \omega_{c}^{3}(x)} \tilde{v} \cdot \tilde{v} \\
& +\frac{1}{2 \pi \varepsilon_{0} B}\left(\operatorname{div}_{x} \int_{\mathbb{R}^{2}} \frac{{ }^{\perp}\left(x-x^{\prime}\right)}{\left|x-x^{\prime}\right|^{2}} \otimes j[\tilde{f}]\left(x^{\prime}\right) \mathrm{d} x^{\prime} \cdot \frac{{ }^{\perp} \tilde{v}}{\omega_{c}(x)}\right) \frac{\tilde{v} \cdot \nabla_{\tilde{v}} \tilde{f}}{|\tilde{v}|^{2}} \\
& -\left(\frac{\partial_{x}\left(\frac{{ }^{\perp} E}{B}\right) \mathcal{R}\left(-\frac{\pi}{2}\right)+\mathcal{R}\left(\frac{\pi}{2}\right) \partial_{x}\left(\frac{{ }^{\perp} E}{B}\right)}{4 \omega_{c}(x)} \tilde{v} \cdot \tilde{v}\right) \frac{\tilde{v} \cdot \nabla_{\tilde{v}} \tilde{f}}{|\tilde{v}|^{2}} .
\end{aligned}
$$

Proof. Notice that

$$
-A_{x}(x, \tilde{v}) \cdot \nabla_{x} \tilde{f}=-\frac{\left({ }^{\perp} \tilde{v} \cdot \nabla_{x} \tilde{f}\right)\left(\tilde{v} \cdot \nabla \omega_{c}\right)}{\omega_{c}^{2}(x)}=\frac{\left(\tilde{v} \cdot{ }^{\perp} \nabla_{x} \tilde{f}\right)\left(\tilde{v} \cdot \nabla \omega_{c}\right)}{\omega_{c}^{2}(x)}=\frac{\nabla \omega_{c} \otimes{ }^{\perp} \nabla_{x} \tilde{f}}{\omega_{c}^{2}(x)} \tilde{v} \cdot \tilde{v}
$$

and therefore, thanks to Lemma 5.1, we have

$$
\begin{aligned}
-A_{x}(x, \tilde{v}) \cdot \nabla_{x} \tilde{f}+\frac{m|\tilde{v}|^{2}}{2 q B(x)} \frac{\perp \nabla B}{B(x)} \cdot \nabla_{x} \tilde{f} & =\frac{\nabla \omega_{c} \otimes{ }^{\perp} \nabla_{x} \tilde{f}}{\omega_{c}^{2}(x)} \tilde{v} \cdot \tilde{v}-\left\langle\frac{\nabla \omega_{c} \otimes{ }^{\perp} \nabla_{x} \tilde{f}}{\omega_{c}^{2}(x)} \tilde{v} \cdot \tilde{v}\right\rangle \\
& =-b \cdot \nabla_{x, \tilde{v}} \tilde{f}_{I}^{2}
\end{aligned}
$$

with

$$
\begin{aligned}
\tilde{f}_{I}^{2} & :=\left(\frac{\nabla \omega_{c} \otimes{ }^{\perp} \nabla_{x} \tilde{f}}{4 \omega_{c}^{3}(x)} \mathcal{R}\left(-\frac{\pi}{2}\right)+\mathcal{R}\left(\frac{\pi}{2}\right) \frac{\nabla \omega_{c} \otimes{ }^{\perp} \nabla_{x} \tilde{f}}{4 \omega_{c}^{3}(x)}\right) \tilde{v} \cdot \tilde{v} \\
& =\frac{\nabla \omega_{c} \otimes \nabla_{x} \tilde{f}-{ }^{\perp} \nabla \omega_{c} \otimes{ }^{\perp} \nabla_{x} \tilde{f}}{4 \omega_{c}^{3}(x)} \tilde{v} \cdot \tilde{v} .
\end{aligned}
$$

Observing that $b \cdot \nabla_{x, \tilde{v}}\left(-{ }^{\perp} \tilde{v} / \omega_{c}\right)=\tilde{v}$ and that $\nabla_{\tilde{v}} \tilde{f}=\frac{\nabla_{\tilde{v}} \tilde{f} \tilde{v}}{|\tilde{v}|^{2}} \tilde{v}$, it is easily seen that

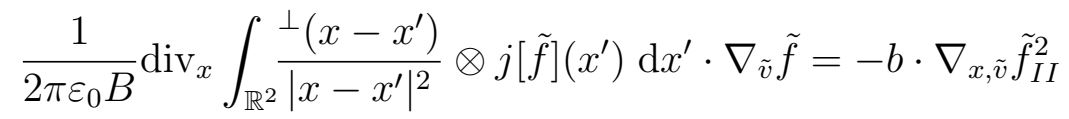


with

$$
\tilde{f}_{I I}^{2}:=\frac{1}{2 \pi \varepsilon_{0} B}\left(\operatorname{div}_{x} \int_{\mathbb{R}^{2}} \frac{\perp\left(x-x^{\prime}\right)}{\left|x-x^{\prime}\right|^{2}} \otimes j[\tilde{f}]\left(x^{\prime}\right) \mathrm{d} x^{\prime} \cdot \frac{{ }^{\perp} \tilde{v}}{\omega_{c}(x)}\right) \frac{\tilde{v} \cdot \nabla_{\tilde{v}} \tilde{f}}{|\tilde{v}|^{2}} .
$$

Using one more time Lemma 5.1, yields

$$
\begin{aligned}
-\partial_{x}\left(\frac{{ }^{\perp} E}{B}\right) \tilde{v} \cdot \nabla_{\tilde{v}} \tilde{f} & -\frac{1}{2}\left(\frac{{ }^{\perp} E}{B} \cdot \frac{\nabla B}{B}\right)\left(\tilde{v} \cdot \nabla_{\tilde{v}} \tilde{f}\right) \\
& =-\frac{\tilde{v} \cdot \nabla_{\tilde{v}} \tilde{f}}{|\tilde{v}|^{2}}\left[\partial_{x}\left(\frac{{ }^{\perp} E}{B}\right) \tilde{v} \cdot \tilde{v}-\left\langle\partial_{x}\left(\frac{{ }^{\perp} E}{B}\right) \tilde{v} \cdot \tilde{v}\right\rangle\right] \\
& =-b \cdot \nabla_{x, \tilde{v}} \tilde{f}_{I I I}^{2}
\end{aligned}
$$

with

$$
\tilde{f}_{I I I}^{2}=-\frac{\tilde{v} \cdot \nabla_{\tilde{v}} \tilde{f}}{|\tilde{v}|^{2}} \frac{\partial_{x}\left(\frac{\perp_{E}}{B}\right) \mathcal{R}\left(-\frac{\pi}{2}\right)+\mathcal{R}\left(\frac{\pi}{2}\right) \partial_{x}\left(\frac{\perp_{E}}{B}\right)}{4 \omega_{c}(x)} \tilde{v} \cdot \tilde{v} .
$$

Our conclusion follows by taking $\tilde{f}^{2}=\tilde{f}_{I}^{2}+\tilde{f}_{I I}^{2}+\tilde{f}_{I I I}^{2}$.

Remark 5.1 Notice that $\tilde{f}^{2} \in C_{c}\left(\mathbb{R}^{2} \times \mathbb{R}^{2}\right)$ and if there is $k \geq 2$ such that $\tilde{f} \in$ $C_{c}^{k}\left(\mathbb{R}^{2} \times \mathbb{R}^{2}\right), E \in C^{k}\left(\mathbb{R}^{2}\right), B \in C^{k}\left(\mathbb{R}^{2}\right)$, then $\tilde{f}^{2} \in C_{c}^{k-1}\left(\mathbb{R}^{2} \times \mathbb{R}^{2}\right)$.

One of the main difficulties when comparing $\tilde{f}^{\varepsilon}, \tilde{f}$ is that these particle densities belong to different constraint spaces. Indeed, the corresponding Vlasov like equations involve different stiff transport terms $\frac{b^{\varepsilon}}{\varepsilon} \cdot \nabla_{x, \tilde{v}}, \frac{b}{\varepsilon} \cdot \nabla_{x, \tilde{v}}$. The idea is to map the above constraint spaces in order to bring $\tilde{f}$ in the constraint space of $\tilde{f}^{\varepsilon}$. In the sequel we will use the map $T^{\varepsilon}: \mathbb{R}^{2} \times \mathbb{R}^{2} \rightarrow \mathbb{R}^{2} \times \mathbb{R}^{2}$, given by

$$
T^{\varepsilon}(x, \tilde{v})=\left(x+\varepsilon \frac{\perp \tilde{v}}{\omega_{c}(x)}, \tilde{v}\right) .
$$

Notice that for any application $\varphi(x, \tilde{v})$ in the kernel of $b \cdot \nabla_{x, \tilde{v}}$ i.e., $\varphi(x, \tilde{v})=\psi(x,|\tilde{v}|)$, the composition product $\varphi \circ T^{\varepsilon}$ depends only on $x+\varepsilon \frac{{ }^{\perp} \tilde{v}}{\omega_{c}(x)},|\tilde{v}|$

$$
\left(\varphi \circ T^{\varepsilon}\right)(x, \tilde{v})=\varphi\left(x+\varepsilon \frac{{ }^{\perp} \tilde{v}}{\omega_{c}(x)}, \tilde{v}\right)=\psi\left(x+\varepsilon \frac{{ }^{\perp} \tilde{v}}{\omega_{c}(x)},|\tilde{v}|\right)
$$

and therefore belongs to the kernel of $b^{\varepsilon} \cdot \nabla_{x, \tilde{v}}$. Actually, by direct computations we check that

$$
\partial_{x, \tilde{v}} T^{\varepsilon} b^{\varepsilon}=\lambda^{\varepsilon} b \circ T^{\varepsilon}, \quad \lambda^{\varepsilon}(x, \tilde{v})=\frac{\omega_{c}(x)}{\omega_{c}(\tilde{x})}, \quad \tilde{x}=x+\varepsilon \frac{{ }^{\perp} \tilde{v}}{\omega_{c}(x)} .
$$

Another difficulty comes by the inhomogeneity of the magnetic field, which accounts for a weight function $\lambda^{\varepsilon}$, close to 1 . We are ready to prove our main result.

Proof. (of Theorem 1.2)

As $g^{\varepsilon}$ belongs to $C_{c}^{2}\left(\mathbb{R}^{2} \times \mathbb{R}^{2}\right)$, it is easily seen that $\left\langle g^{\varepsilon}\right\rangle \in C_{c}^{2}\left(\mathbb{R}^{2} \times \mathbb{R}^{2}\right)$. We deduce by Theorem 1.1 that the restriction on $[0, T]$ of the solution $\tilde{f}$ for $(25),(26)$ corresponding to the initial condition $\left\langle g^{\varepsilon}\right\rangle$ belongs to $C_{c}^{2}\left([0, T] \times \mathbb{R}^{2} \times \mathbb{R}^{2}\right)$. The restriction on $[0, T]$ of the Poisson electric field $E[\tilde{f}]$ belongs to $C^{2}\left([0, T] \times \mathbb{R}^{2}\right)$. As $b \cdot \nabla\left\langle g^{\varepsilon}\right\rangle=0$, we deduce by the last statement in Remark 4.1 that $b \cdot \nabla \tilde{f}(t)=0, t \in \mathbb{R}_{+}$. Applying Proposition 
5.1 with the particle density $\tilde{f}(t) \in C_{c}^{2}\left(\mathbb{R}^{2} \times \mathbb{R}^{2}\right)$, the electric field $E[\tilde{f}(t)] \in C^{2}\left(\mathbb{R}^{2}\right)$, which verify $b \cdot \nabla_{x, \tilde{v}} \tilde{f}(t)=0, \operatorname{div}_{x}{ }^{\perp} E[\tilde{f}(t)]=0$, one gets for any $t \in \mathbb{R}_{+}$

$$
\begin{aligned}
& {\left[\frac{{ }^{\perp} E[\tilde{f}(t)]}{B}-A_{x}\right] \cdot \nabla_{x} \tilde{f}+\left[\frac{\operatorname{div}_{x}}{2 \pi \varepsilon_{0} B} \int_{\mathbb{R}^{2}} \frac{{ }^{\perp}\left(x-x^{\prime}\right)}{\left|x-x^{\prime}\right|^{2}} \otimes j[\tilde{f}]\left(x^{\prime}\right) \mathrm{d} x^{\prime}-\partial_{x}\left(\frac{{ }^{\perp} E[\tilde{f}(t)]}{B}\right) \tilde{v}\right]_{(35)} \nabla_{\tilde{v}} \tilde{f}} \\
& -\left(\frac{{ }^{\perp} E[\tilde{f}(t)]}{B}-\frac{m|\tilde{v}|^{2}}{2 q B} \frac{{ }^{\perp} \nabla B}{B}\right) \cdot \nabla_{x} \tilde{f}-\frac{1}{2}\left(\frac{{ }^{\perp} E[\tilde{f}(t)]}{B} \cdot \frac{\nabla B}{B}\right) \tilde{v} \cdot \nabla_{\tilde{v}} \tilde{f}+b \cdot \nabla_{x, \tilde{v}} \tilde{f}^{2}(t)=0
\end{aligned}
$$

where $\tilde{f}^{2}(t) \in C_{\tilde{c}}^{1}\left(\mathbb{R}^{2} \times \mathbb{R}^{2}\right)$ is given by the formula (33) which corresponds to the particle density $\tilde{f}(t)$ and the electric field $E[\tilde{f}(t)]$. Multiplying (35) by $\varepsilon$ and adding to (25) yield

$$
\partial_{t} \tilde{f}+\varepsilon a[\tilde{f}(t)] \cdot \nabla \tilde{f}(t)+\varepsilon b \cdot \nabla \tilde{f}^{2}(t)=0
$$

and thus the corrector $\tilde{f}^{2}$ allowed us to write the limit model (6) into a form similar to that in (17). Nevertheless the transport operators entering the terms $\frac{b}{\varepsilon} \cdot \nabla\left(\varepsilon^{2} \tilde{f}^{2}\right), \frac{b^{\varepsilon}}{\varepsilon} \cdot \nabla \tilde{f} \varepsilon$ are not exactly the same, which will prevent us for obtaining error estimates by usual $L^{2}$ balances. Therefore we appeal to the map $T^{\varepsilon}$ and weight $\lambda^{\varepsilon}$. Notice that thanks to (34) we can write

$$
\lambda^{\varepsilon}\left(b \cdot \nabla \tilde{f}^{2}\right) \circ T^{\varepsilon}=\partial T^{\varepsilon} b^{\varepsilon} \cdot\left(\nabla \tilde{f}^{2}\right) \circ T^{\varepsilon}=b^{\varepsilon} \cdot \nabla\left(\tilde{f}^{2} \circ T^{\varepsilon}\right)
$$

and therefore, after composition with $T^{\varepsilon}$ and multiplication by $\lambda^{\varepsilon}$, the equation (36) becomes

$$
\lambda^{\varepsilon}\left(\partial_{t} \tilde{f}\right) \circ T^{\varepsilon}+\varepsilon \lambda^{\varepsilon}(a[\tilde{f}(t)] \cdot \nabla \tilde{f}(t)) \circ T^{\varepsilon}+\varepsilon b^{\varepsilon} \cdot \nabla\left(\tilde{f}^{2} \circ T^{\varepsilon}\right)=0
$$

or equivalently

$\partial_{t}\left(\left\langle\lambda^{\varepsilon}\right\rangle \tilde{f} \circ T^{\varepsilon}\right)+\varepsilon \lambda^{\varepsilon}(a[\tilde{f}(t)] \cdot \nabla \tilde{f}(t)) \circ T^{\varepsilon}+\varepsilon b^{\varepsilon} \cdot \nabla\left(\tilde{f}^{2} \circ T^{\varepsilon}\right)=-\varepsilon\left(\left\langle\lambda^{\varepsilon}\right\rangle-\lambda^{\varepsilon}\right)(\langle a\rangle[\tilde{f}(t)] \cdot \nabla \tilde{f}(t)) \circ T^{\varepsilon}$

where we have used the notation

$$
\langle a\rangle[\tilde{f}]:=\left(\frac{{ }^{\perp} E[\tilde{f}]}{B}-\frac{m|\tilde{v}|^{2}}{2 q B} \frac{{ }^{\perp} \nabla B}{B}, \frac{1}{2}\left(\frac{{ }^{\perp} E[\tilde{f}]}{B} \cdot \frac{\nabla B}{B}\right) \tilde{v}\right) .
$$

Notice that, as $\tilde{f}(t)$ belongs to the kernel of $b \cdot \nabla$, then $\tilde{f} \circ T^{\varepsilon}$ belongs to the kernel of $b^{\varepsilon} \cdot \nabla$, as well as $\left\langle\lambda^{\varepsilon}\right\rangle \tilde{f} \circ T^{\varepsilon}$. Combining (17), (37) we obtain

$$
\begin{aligned}
& \partial_{t}\left\{\tilde{f}^{\varepsilon}-\left\langle\lambda^{\varepsilon}\right\rangle \tilde{f} \circ T^{\varepsilon}-\varepsilon^{2} \tilde{f}^{2} \circ T^{\varepsilon}\right\}+\varepsilon a^{\varepsilon}\left[\tilde{f}^{\varepsilon}(t)\right] \cdot \nabla\left(\left\langle\lambda^{\varepsilon}\right\rangle \tilde{f} \circ T^{\varepsilon}\right)-\varepsilon \lambda^{\varepsilon}(a[\tilde{f}(t)] \cdot \nabla \tilde{f}(t)) \circ T^{\varepsilon} \\
& +\left(\varepsilon a^{\varepsilon}\left[\tilde{f}^{\varepsilon}(t)\right]+\frac{b^{\varepsilon}}{\varepsilon}\right) \cdot \nabla\left\{\tilde{f}^{\varepsilon}-\left\langle\lambda^{\varepsilon}\right\rangle \tilde{f} \circ T^{\varepsilon}-\varepsilon^{2} \tilde{f}^{2} \circ T^{\varepsilon}\right\}=-\varepsilon^{2}\left(\partial_{t} \tilde{f}^{2}\right) \circ T^{\varepsilon} \\
& -\varepsilon^{3} a^{\varepsilon}\left[\tilde{f}^{\varepsilon}(t)\right] \cdot \nabla\left(\tilde{f}^{2} \circ T^{\varepsilon}\right)-\varepsilon\left(\lambda^{\varepsilon}-\left\langle\lambda^{\varepsilon}\right\rangle\right)(\langle a\rangle[\tilde{f}(t)] \cdot \nabla \tilde{f}(t)) \circ T^{\varepsilon}
\end{aligned}
$$

By Remark 3.1 we know that $\operatorname{div}\left(\varepsilon a^{\varepsilon}\left[\tilde{f}^{\varepsilon}(t)\right]+b^{\varepsilon} / \varepsilon\right)=0$ and therefore, multiplying (38) by $r^{\varepsilon}:=\tilde{f}^{\varepsilon}-\left\langle\lambda^{\varepsilon}\right\rangle \tilde{f} \circ T^{\varepsilon}-\varepsilon^{2} \tilde{f}^{2} \circ T^{\varepsilon}$ yields

$$
\begin{aligned}
& \frac{1}{2} \frac{\mathrm{d}}{\mathrm{d} t}\left\|r^{\varepsilon}\right\|_{L^{2}}^{2} \leq \varepsilon\left\|r^{\varepsilon}(t)\right\|_{L^{2}}\left\|a^{\varepsilon}\left[\tilde{f}^{\varepsilon}(t)\right] \cdot \nabla\left(\left\langle\lambda^{\varepsilon}\right\rangle \tilde{f} \circ T^{\varepsilon}\right)-\lambda^{\varepsilon}(a[\tilde{f}(t)] \cdot \nabla \tilde{f}(t)) \circ T^{\varepsilon}\right\|_{L^{2}}+\left\|r^{\varepsilon}(t)\right\|_{L^{2}} \\
& \times\left\|\varepsilon^{2}\left(\partial_{t} \tilde{f}^{2}\right) \circ T^{\varepsilon}+\varepsilon^{3} a^{\varepsilon}\left[\tilde{f}^{\varepsilon}(t)\right] \cdot \nabla\left(\tilde{f}^{2} \circ T^{\varepsilon}\right)+\varepsilon\left(\lambda^{\varepsilon}-\left\langle\lambda^{\varepsilon}\right\rangle\right)(\langle a\rangle[\tilde{f}(t)] \cdot \nabla \tilde{f}(t)) \circ T^{\varepsilon}\right\|_{L^{2}} .
\end{aligned}
$$


By Bellman lemma one gets

$$
\begin{aligned}
\left\|r^{\varepsilon}(t)\right\|_{L^{2}} & \leq\left\|r^{\varepsilon}(0)\right\|_{L^{2}}+\varepsilon \int_{0}^{t}\left\|a^{\varepsilon}\left[\tilde{f}^{\varepsilon}(s)\right] \cdot \nabla\left(\left\langle\lambda^{\varepsilon}\right\rangle \tilde{f}(s) \circ T^{\varepsilon}\right)-\lambda^{\varepsilon}(a[\tilde{f}(s)] \cdot \nabla \tilde{f}(s)) \circ T^{\varepsilon}\right\|_{L^{2}} \mathrm{~d} s \\
& +\varepsilon^{2} \int_{0}^{t}\left\|\left(\partial_{t} \tilde{f}^{2}(s)\right) \circ T^{\varepsilon}\right\|_{L^{2}} \mathrm{~d} s+\varepsilon^{3} \int_{0}^{t}\left\|a^{\varepsilon}\left[\tilde{f}^{\varepsilon}(s)\right] \cdot \nabla\left(\tilde{f}^{2}(s) \circ T^{\varepsilon}\right)\right\|_{L^{2}} \mathrm{~d} s \\
& +\varepsilon \int_{0}^{t}\left\|\left(\lambda^{\varepsilon}-\left\langle\lambda^{\varepsilon}\right\rangle\right)(\langle a\rangle[\tilde{f}(s)] \cdot \nabla \tilde{f}(s)) \circ T^{\varepsilon}\right\|_{L^{2}} \mathrm{~d} s .
\end{aligned}
$$

We need to analyze one by one all the terms in the right hand side of the previous inequality, for any $t \in[0, T], T \in \mathbb{R}_{+}$. We will denote by $C$ any constant depending on $m, \varepsilon_{0}, q, T, B$ and the uniform bounds satisfied by the particle densities $\left(g^{\varepsilon}\right)_{\varepsilon>0}$, but not on $\varepsilon$. As $\left(g^{\varepsilon}\right)_{\varepsilon>0}$ are uniformly compactly supported in $\mathbb{R}^{2} \times \mathbb{R}^{2}$ and uniformly bounded in $C^{2}\left(\mathbb{R}^{2} \times \mathbb{R}^{2}\right)$, it is easily seen that so are $\left(\left\langle g^{\varepsilon}\right\rangle\right)_{\varepsilon>0}$. By Theorem 1.1 the solutions $\tilde{f}$ for $(25),(26)$ associated to the initial conditions $\left(\left\langle g^{\varepsilon}\right\rangle\right)_{\varepsilon>0}$, remain uniformly compactly supported in $[0, T] \times \mathbb{R}^{2} \times \mathbb{R}^{2}, T \in \mathbb{R}_{+}$, and uniformly bounded in $C^{2}\left([0, T] \times \mathbb{R}^{2}\right), T \in \mathbb{R}_{+}$. Clearly, at least for $\varepsilon$ small enough $0<\varepsilon \leq \varepsilon_{T}$ we have

$$
\varepsilon^{2}\left\|\tilde{f}^{2}(t) \circ T^{\varepsilon}\right\|_{L^{2}} \leq C \varepsilon^{2}, \quad t \in[0, T] .
$$

As $B \in C^{2}\left(\mathbb{R}^{2}\right), \inf _{x \in \mathbb{R}^{2}}|B(x)|>0$, for any compact set $B\left(R_{x}\right) \times B\left(R_{\tilde{v}}\right)$ there is a constant $C\left(R_{x}, R_{\tilde{v}}\right)$ such that

$$
\left|\frac{\omega_{c}(x)}{\omega_{c}\left(x+\varepsilon^{\perp} \tilde{v} / \omega_{c}(x)\right)}-1+\varepsilon \frac{\nabla \omega_{c}(x) \cdot{ }^{\perp} \tilde{v}}{\omega_{c}^{2}(x)}\right| \leq C\left(R_{x}, R_{\tilde{v}}\right) \varepsilon^{2}, \quad(x, \tilde{v}) \in B\left(R_{x}\right) \times B\left(R_{\tilde{v}}\right)
$$

and by the third statement in Proposition 3.1, we deduce

$$
\left|\left\langle\lambda^{\varepsilon}\right\rangle-1\right|=\left|\left\langle\frac{\omega_{c}(x)}{\omega_{c}(\tilde{x})}-1+\varepsilon \frac{\nabla \omega_{c} \cdot{ }^{\perp} \tilde{v}}{\omega_{c}^{2}}\right\rangle\right| \leq C\left(R_{x}, R_{\tilde{v}}\right) \varepsilon^{2}, \quad(x, \tilde{v}) \in B\left(R_{x}-\varepsilon \frac{R_{\tilde{v}}}{\omega_{0}}\right) \times B\left(R_{\tilde{v}}\right) .
$$

Notice that $\left\|f^{\varepsilon}(0)-g^{\varepsilon}\right\|_{L^{2}}$, and therefore $\left\|\rho\left[f^{\varepsilon}(0)\right]-\rho\left[g^{\varepsilon}\right]\right\|_{L^{2}}$ are of order $\varepsilon$, implying that

$$
\left\|E\left[f^{\varepsilon}(0)\right]-E\left[g^{\varepsilon}\right]\right\|_{L^{2}\left(\mathbb{R}^{2}\right)} \leq C \varepsilon .
$$

We intend to compare $\tilde{f}^{\varepsilon}(0)$ with respect to $\tilde{f}(0) \circ T^{\varepsilon}$. We have

$$
\begin{aligned}
\tilde{f}^{\varepsilon}(0, x, \tilde{v}) & =f^{\varepsilon}\left(0, x, \tilde{v}+\varepsilon \frac{{ }^{\perp} E\left[f^{\varepsilon}(0)\right]}{B(x)}\right) \\
& =g^{\varepsilon}\left(x+{\frac{\varepsilon}{\omega_{c}(x)}}^{\perp}\left(\tilde{v}+\varepsilon \frac{{ }^{\perp} E\left[f^{\varepsilon}(0)\right]}{B(x)}\right), \tilde{v}+\varepsilon \frac{{ }^{\perp} E\left[f^{\varepsilon}(0)\right]}{B(x)}-\varepsilon \frac{{ }^{\perp} E\left[g^{\varepsilon}\right]}{B(x)}\right)
\end{aligned}
$$

and therefore $\left\|\tilde{f}^{\varepsilon}(0)-g^{\varepsilon} \circ T^{\varepsilon}\right\|_{L^{2}} \leq C \varepsilon^{2}$. Combining the previous arguments, together with the inequality

$$
\left\|g^{\varepsilon}-\left\langle g^{\varepsilon}\right\rangle\right\|_{L^{2}} \leq \frac{2 \pi}{\omega_{0}}\left\|b \cdot \nabla g^{\varepsilon}\right\|_{L^{2}} \leq \varepsilon^{2} \frac{2 \pi}{\omega_{0}} \sup _{\eta>0} \frac{\left\|b \cdot \nabla g^{\eta}\right\|_{L^{2}}}{\eta^{2}}
$$


cf. statement 5 in Proposition 3.1, we obtain

$$
\begin{aligned}
\left\|r^{\varepsilon}(0)\right\|_{L^{2}} & \leq\left\|\tilde{f}^{\varepsilon}(0)-\left\langle\lambda^{\varepsilon}\right\rangle \tilde{f}(0) \circ T^{\varepsilon}\right\|_{L^{2}}+\varepsilon^{2}\left\|\tilde{f}^{2}(0) \circ T^{\varepsilon}\right\|_{L^{2}} \\
& \leq\left\|\tilde{f}^{\varepsilon}(0)-g^{\varepsilon} \circ T^{\varepsilon}\right\|_{L^{2}}+\left\|g^{\varepsilon} \circ T^{\varepsilon}-\left\langle g^{\varepsilon}\right\rangle \circ T^{\varepsilon}\right\|_{L^{2}} \\
& +\left\|\left(1-\left\langle\lambda^{\varepsilon}\right\rangle\right)\left\langle g^{\varepsilon}\right\rangle \circ T^{\varepsilon}\right\|_{L^{2}}+\varepsilon^{2}\left\|\tilde{f}^{2}(0) \circ T^{\varepsilon}\right\|_{L^{2}} \leq C \varepsilon^{2}
\end{aligned}
$$

Notice that (40), (41) also imply that $\left(\frac{\lambda^{\varepsilon}-\left\langle\lambda^{\varepsilon}\right\rangle}{\varepsilon}\right)_{\varepsilon>0}$ is bounded on compact sets of $\mathbb{R}^{2} \times \mathbb{R}^{2}$, and thus

$$
\varepsilon \int_{0}^{t}\left\|\left(\lambda^{\varepsilon}-\left\langle\lambda^{\varepsilon}\right\rangle\right)(\langle a\rangle[\tilde{f}(s)] \cdot \nabla \tilde{f}(s)) \circ T^{\varepsilon}\right\|_{L^{2}} \mathrm{~d} s \leq C \varepsilon^{2}
$$

Using the $C_{c}^{1}$ regularity of $\tilde{f}^{2}$, and therefore the $C_{c}^{2}$ regularity of $\tilde{f}$, it is straightforward that

$$
\varepsilon^{2} \int_{0}^{t}\left\|\left(\partial_{t} \tilde{f}^{2}(s)\right) \circ T^{\varepsilon}\right\|_{L^{2}} \mathrm{~d} s+\varepsilon^{3} \int_{0}^{t}\left\|a^{\varepsilon}\left[\tilde{f}^{\varepsilon}(s)\right] \cdot \nabla\left(\tilde{f}^{2}(s) \circ T^{\varepsilon}\right)\right\|_{L^{2}} \mathrm{~d} s \leq C \varepsilon^{2}, \quad t \in[0, T] .
$$

We claim that

$$
\begin{aligned}
\varepsilon \int_{0}^{t} \| a^{\varepsilon}\left[\tilde{f}^{\varepsilon}(s)\right] & \cdot \nabla\left(\left\langle\lambda^{\varepsilon}\right\rangle \tilde{f}(s) \circ T^{\varepsilon}\right)-\lambda^{\varepsilon}(a[\tilde{f}(s)] \cdot \nabla \tilde{f}(s)) \circ T^{\varepsilon} \|_{L^{2}} \leq C \varepsilon^{2} \\
& +C \varepsilon \int_{0}^{t}\left\|\tilde{f}^{\varepsilon}(s)-\tilde{f}(s) \circ T^{\varepsilon}\right\|_{L^{2}} \mathrm{~d} s, \quad t \in[0, T], \quad 0<\varepsilon \leq \varepsilon_{T} .
\end{aligned}
$$

These arguments are technical and come by the smoothness and uniform bounds of the family $\left(\tilde{f}^{\varepsilon}\right)_{\varepsilon>0}$, combined to the asymptotic behavior of $A_{x}^{\varepsilon}, T^{\varepsilon}, \lambda^{\varepsilon}$ when $\varepsilon \searrow 0$. Indeed, recall that for any $(x, \tilde{v}) \in \mathbb{R}^{2} \times B(R)$ and any $\varepsilon>0$ such that $\frac{\varepsilon R\left\|\nabla \omega_{c}\right\|_{L^{\infty}}}{\omega_{0}^{2}} \leq \frac{1}{2}$, we have

$$
\left|A_{x}^{\varepsilon}(x, \tilde{v})\right| \leq 2\left|A_{x}(x, \tilde{v})\right|, \quad\left|A_{x}^{\varepsilon}(x, \tilde{v})-A_{x}(x, \tilde{v})\right| \leq \frac{\varepsilon|\tilde{v}|\left\|\nabla \omega_{c}\right\|_{L^{\infty}}}{\omega_{0}^{2}}\left|A_{x}^{\varepsilon}(x, \tilde{v})\right| \leq \frac{\left|A_{x}^{\varepsilon}(x, \tilde{v})\right|}{2}
$$

implying that

$$
\left|A_{x}^{\varepsilon}(x, \tilde{v})\right| \leq 2\left|A_{x}(x, \tilde{v})\right| \leq \frac{2 R^{2}\left\|\nabla \omega_{c}\right\|_{L^{\infty}}}{\omega_{0}^{2}}, \quad\left|A_{x}^{\varepsilon}(x, \tilde{v})-A_{x}(x, \tilde{v})\right| \leq 2 \varepsilon \frac{R^{3}\left\|\nabla \omega_{c}\right\|_{L^{\infty}}^{2}}{\omega_{0}^{4}} .
$$

As $\left\{\tilde{f}^{\varepsilon}(t), t \in[0, T], 0<\varepsilon \leq 1\right\}$ are uniformly compactly supported in $\mathbb{R}^{2} \times \mathbb{R}^{2}$, and thanks to the uniform bounds

$$
\sup _{\varepsilon>0, t \in[0, T]}\left\{\left\|\tilde{f}^{\varepsilon}(t)\right\|_{C^{1}\left(\mathbb{R}^{2} \times \mathbb{R}^{2}\right)}+\left\|E\left[\tilde{f}^{\varepsilon}(t)\right]\right\|_{C^{1}\left(\mathbb{R}^{2}\right)}\right\}<+\infty
$$

we have

$$
\left\|\left(a^{\varepsilon}\left[\tilde{f}^{\varepsilon}(t)\right]-a\left[\tilde{f}^{\varepsilon}(t)\right]\right) \cdot \nabla\left(\left\langle\lambda^{\varepsilon}\right\rangle \tilde{f}(t) \circ T^{\varepsilon}\right)\right\|_{L^{2}} \leq C \varepsilon, \quad t \in[0, T] .
$$

Using elliptic regularity results, the quantity

$$
\left\|\left(a\left[\tilde{f}^{\varepsilon}(t)\right]-a\left[\tilde{f}(t) \circ T^{\varepsilon}\right]\right) \cdot \nabla\left(\left\langle\lambda^{\varepsilon}\right\rangle \tilde{f}(t) \circ T^{\varepsilon}\right)\right\|_{L^{2}\left(\mathbb{R}^{2} \times \mathbb{R}^{2}\right)}
$$


is bounded by the $L^{2}$ norms of the charge and current densities

$$
\left\|\rho\left[\tilde{f}^{\varepsilon}(t)\right]-\rho\left[\tilde{f}(t) \circ T^{\varepsilon}\right]\right\|_{L^{2}\left(\mathbb{R}^{2}\right)}+\left\|j\left[\tilde{f}^{\varepsilon}(t)\right]-j\left[\tilde{f}(t) \circ T^{\varepsilon}\right]\right\|_{L^{2}\left(\mathbb{R}^{2}\right)}
$$

and thus by the $L^{2}$ norm of the particle densities $\left\|\tilde{f} \varepsilon(t)-\tilde{f}(t) \circ T^{\varepsilon}\right\|_{L^{2}\left(\mathbb{R}^{2} \times \mathbb{R}^{2}\right)}$. Therefore we have the inequality

$\left\|\left(a\left[\tilde{f}^{\varepsilon}(t)\right]-a\left[\tilde{f}(t) \circ T^{\varepsilon}\right]\right) \cdot \nabla\left(\left\langle\lambda^{\varepsilon}\right\rangle \tilde{f}(t) \circ T^{\varepsilon}\right)\right\|_{L^{2}\left(\mathbb{R}^{2} \times \mathbb{R}^{2}\right)} \leq C\left\|\tilde{f}^{\varepsilon}(t)-\tilde{f}(t) \circ T^{\varepsilon}\right\|_{L^{2}\left(\mathbb{R}^{2} \times \mathbb{R}^{2}\right)}, t \in[0, T]$.

We are done if we prove that

$\left\|a\left[\tilde{f}(t) \circ T^{\varepsilon}\right] \cdot \nabla\left(\left\langle\lambda^{\varepsilon}\right\rangle \tilde{f}(t) \circ T^{\varepsilon}\right)-\lambda^{\varepsilon}(a[\tilde{f}(t)] \cdot \nabla \tilde{f}(t)) \circ T^{\varepsilon}\right\|_{L^{2}\left(\mathbb{R}^{2} \times \mathbb{R}^{2}\right)} \leq C \varepsilon, \quad t \in[0, T], \quad \varepsilon>0$

which comes easily by the $C^{2}$ regularity of $\tilde{f}$ and the compactness of its support, and the boundedness of $\left(\frac{\lambda^{\varepsilon}-\left\langle\lambda^{\varepsilon}\right\rangle}{\varepsilon}\right)_{\varepsilon>0},\left(\frac{\nabla\left\langle\lambda^{\varepsilon}\right\rangle}{\varepsilon}\right)_{\varepsilon>0}$ on compact sets of $\mathbb{R}^{2} \times \mathbb{R}^{2}$. We only have to check the boundedness of $\left(\frac{\nabla\left\langle\lambda^{\varepsilon}\right\rangle}{\varepsilon}\right)_{\varepsilon>0}$, because that of $\left(\frac{\lambda^{\varepsilon}-\left\langle\lambda^{\varepsilon}\right\rangle}{\varepsilon}\right)_{\varepsilon>0}$ has been established before. By the statement 6 in Proposition 3.1 we know that

$$
\nabla_{x}\left\langle\lambda^{\varepsilon}\right\rangle=\left\langle\nabla_{x} \lambda^{\varepsilon}\right\rangle, \quad \nabla_{\tilde{v}}\left\langle\lambda^{\varepsilon}\right\rangle=\frac{\tilde{v} \otimes \tilde{v}}{|\tilde{v}|^{2}} \nabla_{\tilde{v}}\left\langle\lambda^{\varepsilon}\right\rangle=\left\langle\frac{\tilde{v} \cdot \nabla_{\tilde{v}} \lambda^{\varepsilon}}{|\tilde{v}|}\right\rangle \frac{\tilde{v}}{|\tilde{v}|} .
$$

Notice that

$$
\nabla_{x} \lambda^{\varepsilon}(x, \tilde{v})=\frac{\nabla \omega_{c}(x)}{\omega_{c}(\tilde{x})}-\frac{\omega_{c}(x)}{\omega_{c}^{2}(\tilde{x})} t\left(I_{2}-\varepsilon \frac{{ }^{\perp} \tilde{v} \otimes \nabla \omega_{c}(x)}{\omega_{c}^{2}(x)}\right) \nabla \omega_{c}(\tilde{x})
$$

and $\left.\nabla_{x} \lambda^{\varepsilon}(x, \tilde{v})\right|_{\varepsilon=0}=0$, implying that $\left(\frac{\nabla_{x}\left\langle\lambda^{\varepsilon}\right\rangle}{\varepsilon}\right)_{\varepsilon>0}$, and therefore $\left(\frac{\left\langle\nabla_{x} \lambda^{\varepsilon}\right\rangle}{\varepsilon}\right)_{\varepsilon>0}=\left(\frac{\nabla_{x}\left\langle\lambda^{\varepsilon}\right\rangle}{\varepsilon}\right)_{\varepsilon>0}$ is bounded on compact sets of $\mathbb{R}^{2} \times \mathbb{R}^{2}$. Similarly we have

$$
\tilde{v} \cdot \nabla_{\tilde{v}} \lambda^{\varepsilon}=-\varepsilon^{\perp} \tilde{v} \cdot \frac{\nabla_{x} \omega_{c}(\tilde{x})}{\omega_{c}^{2}(\tilde{x})}
$$

and therefore

$$
\left|\frac{\nabla_{\tilde{v}}\left\langle\lambda^{\varepsilon}\right\rangle}{\varepsilon}\right|=\left|\frac{\tilde{v} \cdot \nabla_{\tilde{v}}\left\langle\lambda^{\varepsilon}\right\rangle}{\varepsilon} \frac{\tilde{v}}{|\tilde{v}|^{2}}\right|=\left|\frac{\left\langle\tilde{v} \cdot \nabla_{\tilde{v}} \lambda^{\varepsilon}\right\rangle}{\varepsilon} \frac{\tilde{v}}{|\tilde{v}|^{2}}\right| \leq \frac{\left\|\nabla \omega_{c}\right\|_{L^{\infty}}}{\omega_{0}^{2}}, \quad \varepsilon>0 .
$$

Collecting all the previous estimates into (39) leads to the inequality

$$
\begin{aligned}
\left\|\tilde{f}^{\varepsilon}(t)-\tilde{f}(t) \circ T^{\varepsilon}\right\|_{L^{2}} & \leq\left\|r^{\varepsilon}(t)\right\|_{L^{2}}+\left\|\left(\left\langle\lambda^{\varepsilon}\right\rangle-1\right) \tilde{f}(t) \circ T^{\varepsilon}\right\|_{L^{2}}+\varepsilon^{2}\left\|\tilde{f}^{2}(t) \circ T^{\varepsilon}\right\|_{L^{2}} \\
& \leq C \varepsilon^{2}+C \varepsilon \int_{0}^{t}\left\|\tilde{f}^{\varepsilon}(s)-\tilde{f}(s) \circ T^{\varepsilon}\right\|_{L^{2}} \mathrm{~d} s, \quad t \in[0, T], \quad 0<\varepsilon \leq \varepsilon_{T}
\end{aligned}
$$

and by Gronwall lemma we deduce that

$$
\left\|\tilde{f}^{\varepsilon}(t)-\tilde{f}(t) \circ T^{\varepsilon}\right\|_{L^{2}\left(\mathbb{R}^{2} \times \mathbb{R}^{2}\right)} \leq C \varepsilon^{2} \exp (C \varepsilon t), \quad t \in[0, T], \quad 0<\varepsilon \leq \varepsilon_{T} .
$$

In particular, as the $L^{2}$ norm of the Poisson electric field is controlled by the $L^{2}$ norm of the charge density, and therefore by the $L^{2}$ norm of the particle density (because of the compactness of its support), we have

$$
\left\|E\left[\tilde{f}^{\varepsilon}(t)\right]-E\left[\tilde{f}(t) \circ T^{\varepsilon}\right]\right\|_{L^{2}\left(\mathbb{R}^{2}\right)} \leq C \varepsilon^{2}, \quad t \in[0, T], \quad 0<\varepsilon \leq \varepsilon_{T} .
$$


It is easily seen that, thanks to the inequality $\left\|\tilde{f}(t) \circ T^{\varepsilon}-\tilde{f}(t)\right\|_{L^{2}\left(\mathbb{R}^{2} \times \mathbb{R}^{2}\right)} \leq C \varepsilon$ that

$$
\left\|E\left[\tilde{f}(t) \circ T^{\varepsilon}\right]-E[\tilde{f}(t)]\right\|_{L^{2}\left(\mathbb{R}^{2}\right)} \leq C \varepsilon, \quad t \in[0, T]
$$

and therefore

$$
\left\|E\left[f^{\varepsilon}(t)\right]-E[\tilde{f}(t)]\right\|_{L^{2}\left(\mathbb{R}^{2}\right)}=\left\|E\left[\tilde{f}^{\varepsilon}(t)\right]-E[\tilde{f}(t)]\right\|_{L^{2}\left(\mathbb{R}^{2}\right)} \leq C \varepsilon, \quad t \in[0, T], \quad 0<\varepsilon \leq \varepsilon_{T} .
$$

Finally we obtain for any $t \in[0, T], 0<\varepsilon \leq \varepsilon_{T}$

$$
\begin{aligned}
& \left\{\int_{\mathbb{R}^{2}} \int_{\mathbb{R}^{2}}\left[f^{\varepsilon}(t, x, v)-\tilde{f}\left(t, x+\varepsilon \frac{{ }^{\perp} v}{\omega_{c}(x)}, v-\varepsilon \frac{{ }^{\perp} E[\tilde{f}(t)]}{B(x)}\right)\right]^{2} \mathrm{~d} v \mathrm{~d} x\right\}^{1 / 2}=\left\{\int _ { \mathbb { R } ^ { 2 } } \int _ { \mathbb { R } ^ { 2 } } \left[\tilde{f}^{\varepsilon}(t, x, \tilde{v})\right.\right. \\
& \left.\left.-\tilde{f}\left(t, x+\frac{\varepsilon}{\omega_{c}(x)} \perp\left(\tilde{v}+\varepsilon \frac{{ }^{\perp} E\left[f^{\varepsilon}(t)\right]}{B(x)}\right), \tilde{v}+\varepsilon \frac{{ }^{\perp} E\left[f^{\varepsilon}(t)\right]-{ }^{\perp} E[\tilde{f}(t)]}{B(x)}\right)\right]^{2} \mathrm{~d} \tilde{v} \mathrm{~d} x\right\}^{1 / 2} \\
& \leq\left\|\tilde{f}^{\varepsilon}(t)-\tilde{f}(t) \circ T^{\varepsilon}\right\|_{L^{2}\left(\mathbb{R}^{2} \times \mathbb{R}^{2}\right)}+\left\{\int _ { \mathbb { R } ^ { 2 } } \int _ { \mathbb { R } ^ { 2 } } \left[\tilde{f}\left(t, x+\varepsilon \frac{{ }^{\perp} \tilde{v}}{\omega_{c}(x)}, \tilde{v}\right)\right.\right. \\
& \left.\left.-\tilde{f}\left(t, x+\varepsilon \frac{{ }^{\perp} \tilde{v}}{\omega_{c}(x)}-\frac{\varepsilon^{2}}{\omega_{c}(x)} \frac{E\left[f^{\varepsilon}(t)\right]}{B(x)}, \tilde{v}+\varepsilon \frac{{ }^{\perp} E\left[f^{\varepsilon}(t)\right]-{ }^{\perp} E[\tilde{f}(t)]}{B(x)}\right)\right]^{2} \mathrm{~d} \tilde{v} \mathrm{~d} x\right\}^{1 / 2} \\
& \leq C \varepsilon^{2}+C \varepsilon\left\|E\left[f^{\varepsilon}(t)\right]-E[\tilde{f}(t)]\right\|_{L^{2}\left(\mathbb{R}^{2}\right)} \leq C \varepsilon^{2} .
\end{aligned}
$$

\section{Toward more general results}

When the magnetic field is uniform, it is possible to go further in our analysis, by considering smooth initial particle densities, not necessarily well prepared. We mention that most of the studies concentrate only on models with well prepared initial particle densities. We intend to extend the previous analysis for general initial conditions. The asymptotic behavior is more complicated because the particle densities $\left(\tilde{f}^{\varepsilon}\right)_{\varepsilon>0}$ present fast oscillations in time. We appeal to a two scale approach by working in a extended phase space supplemented by a fast time variable. For any non negative initial particle density $f_{\text {in }} \in C_{c}^{2}\left(\mathbb{R}^{2} \times \mathbb{R}^{2}\right)$, let us denote by $f^{\varepsilon} \in C^{2}\left(\mathbb{R}_{+} \times \mathbb{R}^{2} \times \mathbb{R}^{2}\right)$ the solution of (1), (2), with $E\left[f^{\varepsilon}\right] \in C^{2}\left(\mathbb{R}_{+} \times \mathbb{R}^{2}\right)$, where the magnetic field writes $B^{\varepsilon}=\frac{B}{\varepsilon}$, for some constant $B \neq 0$. As before, the change of coordinates (12), (13) leads to the problem

$$
\begin{gathered}
\partial_{t} \tilde{f}^{\varepsilon}+\varepsilon a^{\varepsilon}\left[\tilde{f}^{\varepsilon}(t)\right] \cdot \nabla_{x, \tilde{v}} \tilde{f}^{\varepsilon}+\frac{b^{\varepsilon}(x, \tilde{v})}{\varepsilon} \cdot \nabla_{x, \tilde{v}} \tilde{f}^{\varepsilon}=0, \quad(t, x, \tilde{v}) \in \mathbb{R}_{+} \times \mathbb{R}^{2} \times \mathbb{R}^{2} \\
\tilde{f}^{\varepsilon}(0, x, \tilde{v})=f_{\text {in }}\left(x, \tilde{v}+\varepsilon \frac{{ }^{\perp} E\left[f_{\text {in }}\right](x)}{B}\right), \quad(x, \tilde{v}) \in \mathbb{R}^{2} \times \mathbb{R}^{2} .
\end{gathered}
$$

Notice that there is no correction $A_{x}^{\varepsilon}$ when the magnetic field is uniform, since in that case the solution of (18) is $A_{x}^{\varepsilon}=0$. The characteristic flow of the vector field $b^{\varepsilon} \cdot \nabla_{x, \tilde{v}}=\varepsilon \tilde{v} \cdot \nabla_{x}+\omega_{c}{ }^{\perp} \tilde{v} \cdot \nabla_{\tilde{v}}$ is given by

$$
X^{\varepsilon}(s ; x, \tilde{v})=x+\varepsilon\left[I_{2}-\mathcal{R}\left(-\omega_{c} s\right)\right] \frac{{ }^{\perp} v}{\omega_{c}}, \quad \tilde{\mathcal{V}}^{\varepsilon}(s ; x, \tilde{v})=\mathcal{R}\left(-\omega_{c} s\right) \tilde{v}, \omega_{c}=\frac{q B}{m} .
$$


In order to filter out the fast oscillations corresponding to the vector field $\frac{b^{\varepsilon}(x, \tilde{v})}{\varepsilon} \cdot \nabla_{x, \tilde{v}}$, we perform one more change of coordinates

$$
\tilde{f}^{\varepsilon}(t, x, \tilde{v})=\tilde{F}^{\varepsilon}(t, X, \tilde{V}), \quad(X, \tilde{V})=\left(X^{\varepsilon}, \tilde{\mathcal{V}}^{\varepsilon}\right)(-t / \varepsilon ; x, \tilde{v}) .
$$

By applying the chain rule, we obtain

$$
\partial_{t} \tilde{F}^{\varepsilon}+\varepsilon \partial_{x, \tilde{v}}\left(X^{\varepsilon}, \tilde{\mathcal{V}}^{\varepsilon}\right)\left(-t / \varepsilon ;\left(X^{\varepsilon}, \tilde{\mathcal{V}}^{\varepsilon}\right)(t / \varepsilon ; X, \tilde{V})\right) a^{\varepsilon}\left[\tilde{F}^{\varepsilon}(t)_{-t / \varepsilon}\right]_{t / \varepsilon} \cdot \nabla_{X, \tilde{V}} \tilde{F}^{\varepsilon}=0
$$

where we have used the notations

$$
\tilde{F}^{\varepsilon}(t)_{-t / \varepsilon}=\tilde{F}^{\varepsilon}(t) \circ\left(X^{\varepsilon}, \tilde{\mathcal{V}}^{\varepsilon}\right)\left(-\frac{t}{\varepsilon}\right), a^{\varepsilon}\left[\tilde{F}^{\varepsilon}(t)_{-t / \varepsilon}\right]_{t / \varepsilon}=a^{\varepsilon}\left[\tilde{F}^{\varepsilon}(t) \circ\left(X^{\varepsilon}, \tilde{\mathcal{V}}^{\varepsilon}\right)\left(-\frac{t}{\varepsilon}\right)\right] \circ\left(X^{\varepsilon}, \tilde{\mathcal{V}}^{\varepsilon}\right)\left(\frac{t}{\varepsilon}\right) .
$$

As the characteristic flow $\left(X^{\varepsilon}, \tilde{\nu}^{\varepsilon}\right)$ in $(43)$ is linear, the jacobian matrix simply writes for any $(x, \tilde{v}) \in \mathbb{R}^{2} \times \mathbb{R}^{2}$

$$
\partial_{x, \tilde{v}}\left(X^{\varepsilon}, \tilde{\mathcal{V}}^{\varepsilon}\right)(-t / \varepsilon ; x, \tilde{v})=\left(\begin{array}{cc}
I_{2} & \frac{\varepsilon}{\omega_{c}} \mathcal{R}(-\pi / 2)\left[I_{2}-\mathcal{R}\left(\omega_{c} t / \varepsilon\right)\right] \\
O_{2} & \mathcal{R}\left(\omega_{c} t / \varepsilon\right)
\end{array}\right)
$$

and therefore (45) becomes

$$
\begin{aligned}
\partial_{t} \tilde{F}^{\varepsilon} & +\varepsilon\left\{a_{x}^{\varepsilon}\left[\tilde{F}^{\varepsilon}(t)_{-t / \varepsilon}\right]_{t / \varepsilon}+\frac{\varepsilon}{\omega_{c}} \mathcal{R}(-\pi / 2)\left[I_{2}-\mathcal{R}\left(\omega_{c} t / \varepsilon\right)\right] a_{\tilde{v}}^{\varepsilon}\left[\tilde{F}^{\varepsilon}(t)_{-t / \varepsilon}\right]_{t / \varepsilon}\right\} \cdot \nabla_{X} \tilde{F}^{\varepsilon} \\
& +\varepsilon \mathcal{R}\left(\omega_{c} t / \varepsilon\right) a_{\tilde{v}}^{\varepsilon}\left[\tilde{F}^{\varepsilon}(t)_{-t / \varepsilon}\right]_{t / \varepsilon} \cdot \nabla_{\tilde{V}} \tilde{F}^{\varepsilon}=0, \quad(t, X, \tilde{V}) \in \mathbb{R}_{+} \times \mathbb{R}^{2} \times \mathbb{R}^{2} .
\end{aligned}
$$

We have obtained a two scale problem and we expect that the asymptotic behavior when $\varepsilon$ becomes small will follow by averaging with respect to the fast time variable $s=t / \varepsilon$. After several computations, up to second order terms, we are led to the model

$\partial_{t} \tilde{F}+\varepsilon \frac{{ }^{\perp} E[\tilde{F}(t)]}{B} \cdot \nabla_{X} \tilde{F}+\varepsilon \frac{{ }^{\perp}\{j[\tilde{F}(t)]-\rho[\tilde{F}(t)] \tilde{V}\}}{2 \varepsilon_{0} B} \cdot \nabla_{\tilde{V}} \tilde{F}=0,(t, X, \tilde{V}) \in \mathbb{R}_{+} \times \mathbb{R}^{2} \times \mathbb{R}^{2}$

which is supplemented by the initial condition

$$
\tilde{F}(0, X, \tilde{V})=f_{\text {in }}\left(X-\varepsilon \frac{{ }^{\perp} \tilde{V}}{\omega_{c}}, \tilde{V}+\varepsilon \frac{{ }^{\perp} E\left[f_{\text {in }}\right](X)}{B}\right), \quad(X, \tilde{V}) \in \mathbb{R}^{2} \times \mathbb{R}^{2}
$$

We expect that solving (46) together with the initial condition (47), will provide a second order approximation for (1), (2). Although the above solution depends on $\varepsilon$, we use the notation $\tilde{F}$, saying that it is an approximation, when $\varepsilon$ becomes small. The well posedness of the limit model (46), (47) is a direct consequence of the well posedness of the vorticity formulation for the $2 D$ incompressible Euler equations, see also Lemma 3.3 [30]. The solution of (46), (47) will allow us to describe the asymptotic behavior of the family $\left(f^{\varepsilon}\right)_{\varepsilon>0}$ corresponding to the initial condition $f_{\text {in }}$, when $\varepsilon$ becomes small, see [9] for the proof details.

Theorem 6.1 Let $f_{\text {in }} \in C_{c}^{2}\left(\mathbb{R}^{2} \times \mathbb{R}^{2}\right)$ be a non negative, smooth, compactly supported particle density. We denote by $\left(f^{\varepsilon}\right)_{\varepsilon>0}$ the solutions of the Vlasov-Poisson equations 
(1), (2) with uniform external magnetic field $\frac{B}{\varepsilon} \neq 0$, corresponding to the initial condition $f_{\text {in }}$. Then for any $T \in \mathbb{R}_{+}$, there is a constant $C_{T}>0$ such that for any $\varepsilon>0$

$$
\sup _{t \in[0, T]}\left\{\int_{\mathbb{R}^{2}} \int_{\mathbb{R}^{2}}\left[f^{\varepsilon}(t, x, v)-\tilde{F}\left(t, x+\varepsilon \frac{{ }^{\perp} v}{\omega_{c}}, \mathcal{R}\left(\omega_{c} \frac{t}{\varepsilon}\right)\left(v-\varepsilon \frac{{ }^{\perp} E[\tilde{F}(t)]}{B}\right)\right)\right]^{2} \mathrm{~d} v \mathrm{~d} x\right\}^{1 / 2} \leq C_{T} \varepsilon^{2}
$$

where $\tilde{F}$ is the solution of (46), (47).

The previous results extend to the three dimensional Vlasov-Poisson system, when the curvature of the magnetic lines is not neglected anymore. The regular reformulations of this problem will be the topic of a future work [10,11]. The previous method provides limit models for both well prepared and general initial particle densities. Indeed, following the same steps, it is possible to emphasize a periodic fast dynamics, preserving the guiding center. Averaging with respect to this fast dynamics leads to regular reformulations taking into account all the curvature effects of the magnetic field. We mention that few mathematical studies investigate the three dimensional setting, with curved magnetic fields. We intend to study the well posedness of these reformulations and to establish second order estimates, following the technique employed in the two dimensional framework.

Another important direction will be to investigate models with self-consistent magnetic field, that is, to consider the Vlasov-Maxwell equations with strong external magnetic field. It will be very interesting to derive, at least formally, the effective equations, up to second order terms. Taking into account the effect of collisions, or the mass ratio between ions and electrons, could be tackled by similar arguments.

A very interesting issue will concern the comparison of the above models with respect to the results obtained by plasma physicists. As in the two dimensional setting, the periodic framework is extremely favorable, since it allows us to get completely explicit models. Nevertheless the analysis is much more difficult due to the combination between the parallel and perpendicular dynamics and to the curvature effects. A preliminary work in the two dimensional setting, by neglecting the curvature of the magnetic lines, was a very instructive starting point in order to tackle the general three dimensional setting. We also mention that these arguments, leading to similar models as those in the plasma physics literature [13, 25, 26], are based on standard results in the theory of PDEs. Indeed, our methods do not appeal to notions of hamiltonian mechanics and thus they are accessible to readers which are not familiar with the hamiltonian formalism.

\section{A Well posedness of the Vlasov-Poisson problem with external magnetic field}

Proof. (of Theorem 2.1)

We are done if we prove the well posedness on any time interval $[0, T], T \in \mathbb{R}_{+}$. We construct a map acting on electric fields. Given a $C^{1}$ electric field $E$ on $[0, T] \times \mathbb{R}^{2}$ such that $E, \partial_{x} E$ are bounded, we consider the solution by characteristics of the Vlasov problem $(7)$, (9) on $[0, T] \times \mathbb{R}^{2}$, that is

$$
f^{E}(t, x, v)=f_{\text {in }}\left(X^{E}(0 ; t, x, v), V^{E}(0 ; t, x, v)\right), \quad(t, x, v) \in[0, T] \times \mathbb{R}^{2} \times \mathbb{R}^{2}
$$


where the characteristics $\left(X^{E}, V^{E}\right)(s ; t, x, v)$ are given by

$$
\begin{gathered}
\frac{\mathrm{d} X^{E}}{\mathrm{~d} s}=V^{E}(s), \quad \frac{\mathrm{d} V^{E}}{\mathrm{~d} s}=\frac{q}{m}\left(E\left(s, X^{E}(s)\right)+B\left(X^{E}(s)\right){ }^{\perp} V^{E}(s)\right) \\
\left(X^{E}, V^{E}\right)(s=t ; t, x, v)=(x, v), \quad(s, t, x, v) \in[0, T] \times[0, T] \times \mathbb{R}^{2} \times \mathbb{R}^{2} .
\end{gathered}
$$

Notice that the right hand side terms in the characteristic equations have at most linear growth at infinity (i.e., when $s, t \in[0, T],|x|+|v| \rightarrow+\infty$ ), and therefore the characteristics exist globally on $[0, T]$ and have $C^{1}$ regularity in all the arguments. Considering now the Poisson electric field corresponding to the particle density $f$, leads to a map $\mathcal{F}$, whose fixed point gives the solution of the Vlasov-Poisson problem (7), (8), (9) on $[0, T]$

$$
E \rightarrow \mathcal{F}(E)=\frac{q}{2 \pi \varepsilon_{0}} \int_{\mathbb{R}^{2}} \int_{\mathbb{R}^{2}} f^{E}\left(t, x^{\prime}, v^{\prime}\right) \frac{x-x^{\prime}}{\left|x-x^{\prime}\right|^{2}} \mathrm{~d} v^{\prime} \mathrm{d} x^{\prime}
$$

We need to find a set $X_{T}$ of smooth electric fields, which is left invariant by the map $\mathcal{F}$, and to establish an estimate like

$$
\|\mathcal{F} E(t)-\mathcal{F} \tilde{E}(t)\|_{L^{\infty}\left(\mathbb{R}^{2}\right)} \leq C_{T} \int_{0}^{t}\|E(s)-\tilde{E}(s)\|_{L^{\infty}\left(\mathbb{R}^{2}\right)} \mathrm{d} s, \quad E, \tilde{E} \in X_{T}, t \in[0, T]
$$

for some constant $C_{T}$, not depending on $E, \tilde{E}$. After that, the well posedness of the Vlasov-Poisson system follows immediately, by iterating the map $\mathcal{F}$. We are not indicating all the details of this construction, but only the a priori estimates, for smooth solutions of (7), (8), (9). Let $f$ be a smooth solution corresponding to the non negative initial particle density $f_{\text {in }} \in C_{c}^{1}\left(\mathbb{R}^{2} \times \mathbb{R}^{2}\right)$. We are looking for estimating $E, \partial_{x} E$ in $C\left([0, T] \times \mathbb{R}^{2}\right)$. Notice that for any $R>0$ we have

$$
\begin{aligned}
\left|\varepsilon_{0} E[f(t)](x)\right| & \leq \frac{1}{2 \pi} \int_{\mathbb{R}^{2}} \mathbf{1}_{\left\{\left|x-x^{\prime}\right|<R\right\}} \frac{\rho[f(t)]\left(x^{\prime}\right)}{\left|x-x^{\prime}\right|} \mathrm{d} x^{\prime}+\frac{1}{2 \pi} \int_{\mathbb{R}^{2}} \mathbf{1}_{\left\{\left|x-x^{\prime}\right| \geq R\right\}} \frac{\rho[f(t)]\left(x^{\prime}\right)}{\left|x-x^{\prime}\right|} \mathrm{d} x^{\prime} \\
& \leq R\|\rho[f(t)]\|_{L^{\infty}\left(\mathbb{R}^{2}\right)}+\frac{1}{2 \pi R}\|\rho[f(t)]\|_{L^{1}\left(\mathbb{R}^{2}\right)} .
\end{aligned}
$$

As the total charge is conserved, after minimization with respect to $R$, that is by taking $R=\left(\left\|\rho\left[f_{\text {in }}\right]\right\|_{L^{1}\left(\mathbb{R}^{2}\right)} / 2 \pi\|\rho[f(t)]\|_{L^{\infty}\left(\mathbb{R}^{2}\right)}\right)^{1 / 2}$, we obtain

$$
\varepsilon_{0}\|E[f(t)]\|_{L^{\infty}\left(\mathbb{R}^{2}\right)} \leq\|\rho[f(t)]\|_{L^{\infty}\left(\mathbb{R}^{2}\right)}^{1 / 2}\left(\frac{|q|}{2 \pi}\left\|f_{\mathrm{in}}\right\|_{L^{1}\left(\mathbb{R}^{2} \times \mathbb{R}^{2}\right)}\right)^{1 / 2} .
$$

For estimating the $L^{\infty}$ norm of the charge density, we analyze the support of the particle density $f$. By the characteristic equations we have for any $0 \leq s \leq t \leq T$

$$
|X(s ; t, x, v)-x| \leq \int_{s}^{t}|V(\sigma ; t, x, v)| \mathrm{d} \sigma
$$

and

$$
\frac{1}{2} \frac{\mathrm{d}}{\mathrm{d} s}|V(s ; t, x, v)|^{2}=\frac{q}{m} E[f(s)](X(s ; t, x, v)) \cdot V(s ; t, x, v)
$$


implying that

$$
|| V(s ; t, x, v)|-| v|| \leq \frac{|q|}{m} \int_{s}^{t}\|E[f(\sigma)]\|_{L^{\infty}\left(\mathbb{R}^{2}\right)} \mathrm{d} \sigma .
$$

Assume that $\operatorname{supp} f_{\text {in }} \subset\left\{(x, v) \in \mathbb{R}^{2} \times \mathbb{R}^{2}:|x| \leq R_{x}^{\text {in }}\right.$ and $\left.|v| \leq R_{v}^{\text {in }}\right\}$. Clearly, for any $(x, v) \in \mathbb{R}^{2} \times \mathbb{R}^{2}$ such that $|v|>R_{v}(t):=R_{v}^{\text {in }}+\frac{|q|}{m} \int_{0}^{t}\|E[f(s)]\|_{L^{\infty}\left(\mathbb{R}^{2}\right)} \mathrm{d} s$ we have

$$
|V(0 ; t, x, v)| \geq|v|-\frac{|q|}{m} \int_{0}^{t}\|E[f(s)]\|_{L^{\infty}\left(\mathbb{R}^{2}\right)} \mathrm{d} s>R_{v}^{\text {in }}
$$

and therefore

$$
f(t, x, v)=f_{\text {in }}(X(0 ; t, x, v), V(0 ; t, x, v))=0 .
$$

Consider now $(x, v) \in \mathbb{R}^{2} \times \mathbb{R}^{2}$ such that

$$
|x|>R_{x}(t):=R_{x}^{\text {in }}+t R_{v}(t)+\int_{0}^{t} \frac{|q|}{m} \int_{s}^{t}\|E[f(\sigma)]\|_{L^{\infty}\left(\mathbb{R}^{2}\right)} \mathrm{d} \sigma \mathrm{d} s .
$$

If $|v|>R_{v}(t)$ we already know that $f(t, x, v)=0$. If $|v| \leq R_{v}(t)$, we have by (50), (51)

$$
\begin{aligned}
|X(0 ; t, x, v)| & \geq|x|-\int_{0}^{t}|V(s ; t, x, v)| \mathrm{d} s \\
& \geq|x|-\int_{0}^{t}\left[|v|+\frac{|q|}{m} \int_{s}^{t}\|E[f(\sigma)]\|_{L^{\infty}\left(\mathbb{R}^{2}\right)} \mathrm{d} \sigma\right] \mathrm{d} s \\
& \geq|x|-t R_{v}(t)-\int_{0}^{t} \frac{|q|}{m} \int_{s}^{t}\|E[f(\sigma)]\|_{L^{\infty}\left(\mathbb{R}^{2}\right)} \mathrm{d} \sigma \mathrm{d} s>R_{x}^{\text {in }}
\end{aligned}
$$

implying that $f(t, x, v)=f_{\text {in }}(X(0 ; t, x, v), V(0 ; t, x, v))=0$. We have proved that $f$ is compactly supported

$$
\operatorname{supp} f(t) \subset\left\{(x, v) \in \mathbb{R}^{2} \times \mathbb{R}^{2}:|x| \leq R_{x}(t) \text { and }|v| \leq R_{v}(t)\right\}, \quad t \in[0, T] .
$$

Notice that the above computations are not depending on the magnetic field $B$; more exactly the equations (51) will be the same when considering an external magnetic field or not. This is why the arguments used for the Vlasov-Poisson problem also apply for the Vlasov-Poisson problem with external magnetic field. The charge density is bounded by

$$
|\rho[f(t)](x)|=|q| \int_{\mathbb{R}^{2}} f_{\text {in }}(X(0 ; t, x, v), V(0 ; t, x, v)) \mathrm{d} v \leq|q|\left\|f_{\text {in }}\right\|_{L^{\infty}\left(\mathbb{R}^{2} \times \mathbb{R}^{2}\right)} \pi R_{v}^{2}(t) .(52
$$

The a priori bound for the $L^{\infty}$ norm of the electric field comes by Gronwall lemma. Actually, combining (49), (52) yields

$$
\begin{aligned}
\varepsilon_{0}\|E[f(t)]\|_{L^{\infty}\left(\mathbb{R}^{2}\right)} & \leq\left(\frac{|q|}{2 \pi}\left\|f_{\text {in }}\right\|_{L^{1}\left(\mathbb{R}^{2} \times \mathbb{R}^{2}\right)}\right)^{1 / 2}\left(|q|\left\|f_{\text {in }}\right\|_{L^{\infty}\left(\mathbb{R}^{2} \times \mathbb{R}^{2}\right)} \pi\right)^{1 / 2} R_{v}(t) \\
& =\frac{|q|}{\sqrt{2}}\left\|f_{\text {in }}\right\|_{L^{1}\left(\mathbb{R}^{2} \times \mathbb{R}^{2}\right)}^{1 / 2}\left\|f_{\text {in }}\right\|_{L^{\infty}\left(\mathbb{R}^{2} \times \mathbb{R}^{2}\right)}^{1 / 2}\left(R_{v}^{\text {in }}+\frac{|q|}{m} \int_{0}^{t}\|E[f(s)]\|_{L^{\infty}\left(\mathbb{R}^{2}\right)} \mathrm{d} s\right) .
\end{aligned}
$$

Notice that once that we have obtained a $L^{\infty}$ bound for the electric field, we also get a bound for the support size of the particle density $f$. The derivation of the $L^{\infty}$ a priori 
estimate for $\partial_{x} E$ is more elaborated. It was shown in [2] that there is a constant $\tilde{C}$ (depending only on $m, \varepsilon_{0}$ ) such that

$$
\left\|\partial_{x} E[f(t)]\right\|_{L^{\infty}} \leq \tilde{C}\left[\left(1+\|\rho[f(t)]\|_{L^{\infty}}\right)\left(1+\ln ^{+}\left\|\nabla_{x} \rho[f(t)]\right\|_{L^{\infty}}\right)+\|\rho[f(t)]\|_{L^{1}}\right] .
$$

Here the notation $\ln ^{+}$stands for the positive part of $\ln$. We already have a priori bounds for the $L^{\infty}$ norm of $\rho[f(t)]$ (use the estimate for the size of the support of $f$ ) and for the $L^{1}$ norm of $\rho[f(t)]$ (use the conservation of the total charge), and therefore (53) becomes

$$
\left\|\partial_{x} E[f(t)]\right\|_{L^{\infty}} \leq C_{1}\left(1+\ln ^{+}\left\|\nabla_{x} \rho[f(t)]\right\|_{L^{\infty}}\right), \quad t \in[0, T]
$$

for some constant $C_{1}$ depending on $m, \varepsilon_{0}, q, T$. We need to estimate the $L^{\infty}$ norm of $\nabla_{x} \rho[f(t)]$ which writes

$$
\begin{aligned}
\nabla_{x} \rho[f(t)] & =q \nabla_{x} \int_{\mathbb{R}^{2}} f_{\text {in }}(X(0 ; t, x, v), V(0 ; t, x, v)) \mathrm{d} v \\
& =q \int_{\mathbb{R}^{2}} \mathbf{1}_{\left\{|v| \leq R_{v}(t)\right\}}{ }^{t} \partial_{x} X(0 ; t, x, v)\left(\nabla_{X} f_{\text {in }}\right)(X(0 ; t, x, v), V(0 ; t, x, v)) \mathrm{d} v \\
& +q \int_{\mathbb{R}^{2}} \mathbf{1}_{\left\{|v| \leq R_{v}(t)\right\}}{ }^{t} \partial_{x} V(0 ; t, x, v)\left(\nabla_{V} f_{\text {in }}\right)(X(0 ; t, x, v), V(0 ; t, x, v)) \mathrm{d} v
\end{aligned}
$$

A straightforward computation on the characteristic equations shows that there is a constant $C_{2}\left(m, q, T,\|B\|_{W^{1, \infty}}\right) \geq 1$ such that for any $(x, v) \in \mathbb{R}^{2} \times \mathbb{R}^{2},|v| \leq R_{v}(t)$, we have

$$
\left|\partial_{x} X(0 ; t, x, v)\right|+\left|\partial_{x} V(0 ; t, x, v)\right| \leq C_{2} \exp \left(\int_{0}^{t}\left\|\partial_{x} E[f(s)]\right\|_{L^{\infty}} \mathrm{d} s\right), \quad t \in[0, T] .
$$

Coming back to (55) we obtain

$$
\left\|\nabla_{x} \rho[f(t)]\right\|_{L^{\infty}} \leq C_{3} \exp \left(\int_{0}^{t}\left\|\partial_{x} E[f(s)]\right\|_{L^{\infty}} \mathrm{d} s\right), \quad t \in[0, T]
$$

for some constant $C_{3}\left(m, q, T,\|B\|_{W^{1, \infty}}\right) \geq 1$, and therefore

$$
\ln ^{+}\left\|\nabla_{x} \rho[f(t)]\right\|_{L^{\infty}} \leq \ln C_{3}+\int_{0}^{t}\left\|\partial_{x} E[f(s)]\right\|_{L^{\infty}} \mathrm{d} s, \quad t \in[0, T]
$$

Finally, combining with (54) yields

$$
\left\|\partial_{x} E[f(t)]\right\|_{L^{\infty}} \leq C_{4}\left(1+\int_{0}^{t}\left\|\partial_{x} E[f(s)]\right\|_{L^{\infty}} \mathrm{d} s\right), \quad t \in[0, T]
$$

and the a priori estimate for the $L^{\infty}$ norm of $\partial_{x} E[f]$ follows by Gronwall lemma. Based on the $L^{\infty}$ estimates for the electric field together with its spatial derivatives, and also on the compactness of the particle density support, the inequality (48) comes immediately. 


\section{Acknowledgement}

This work has been carried out within the framework of the EUROfusion Consortium and has received funding from the Euratom research and training programme 20142018 under grant agreement No 633053. The views and opinions expressed herein do not necessarily reflect those of the European Commission.

This work has been carried out thanks to the support of the A*MIDEX project (no ANR-11-IDEX-0001-02) funded by the Investissements d'Avenir French Government program, managed by the French National Research Agency (ANR).

\section{References}

[1] A.A. Arsen'ev, Global existence of weak solution of Vlasov's system of equations, Z. Vychisl. Mat. Fiz. 15(1975) 136-147.

[2] J. Batt, Global symmetric solutions of the initial value problem in stellar dynamics, J. Differential Equations 25(1977) 342-364.

[3] M. Bostan, Transport equations with disparate advection fields. Application to the gyrokinetic models in plasma physics, J. Differential Equations 249(2010) 16201663.

[4] M. Bostan, The Vlasov-Maxwell system with strong initial magnetic field. Guiding-center approximation, SIAM J. Multiscale Model. Simul. 6(2007) 10261058.

[5] M. Bostan, Gyro-kinetic Vlasov equation in three dimensional setting. Second order approximation, SIAM J. Multiscale Model. Simul. 8(2010) 1923-1957.

[6] M. Bostan, A. Finot, M. Hauray, The effective Vlasov-Poisson system for strongly magnetized plasmas, C. R. Acad. Sci. Paris, Ser. I 354(2016) 771-777.

[7] M. Bostan, A. Finot, The effective Vlasov-Poisson system for the finite Larmor radius regime, SIAM J. Multiscale Model. Simul. 14(2016) 1238-1275.

[8] M. Bostan, Multi-scale analysis for linear first order PDEs. The finite Larmor radius regime, SIAM J. Math. Anal. 48(2016) 2133-2188.

[9] M. Bostan, Asymptotic behavior for the Vlasov-Poisson equations with strong uniform magnetic field and general initial conditions, in preparation.

[10] M. Bostan, Asymptotic behavior for the Vlasov-Poisson equations with strong external curved magnetic field. Part I : well prepared initial conditions, in preparation.

[11] M. Bostan, Asymptotic behavior for the Vlasov-Poisson equations with strong external curved magnetic field. Part II : general initial conditions, in preparation.

[12] Y. Brenier, Convergence of the Vlasov-Poisson system to the incompressible Euler equations, Comm. Partial Differential Equations 25(2000) 737-754. 
[13] A. Brizard, T.S. Hahm, Foundations of nonlinear gyrokinetic theory. Rev. Mod. Phys. 79(2007) 421-468.

[14] N. Crouseilles, M. Lemou, F. Méhats, Asymptotic preserving schemes for highly oscillatory Vlasov-Poisson equations, J. Comput. Phys. 248(2013) 287-308.

[15] P. Degond, F. Filbet, On the asymptotic limit of the three dimensional VlasovPoisson system for large magnetic field: formal derivation, J. Stat. Phys. 165(2016) 765-784.

[16] P. Degond, A. Lozinski, J. Narski, C. Negulescu, An asymptotic-preserving method for highly anisotropic elliptic equations based on a micro-macro decomposition. J. Comput. Phys. 231(2012) 2724-2740.

[17] F. Filbet, L. Rodrigues, Asymptotically preserving particle-in-cell methods for inhomogeneous strongly magnetized plasmas, SIAM J. Numer. Anal. 55(2017) 2416-2443.

[18] F. Filbet, S. Jin, A class of asymptotic-preserving schemes for kinetic equations and related problems with stiff sources, J. Comput. Phys. 229(2010) 7625-7648.

[19] E. Frénod, E. Sonnendrücker, Homogenization of the Vlasov equation and of the Vlasov-Poisson system with strong external magnetic field, Asymptotic Anal. 18(1998) 193-213.

[20] E. Frénod, E. Sonnendrücker, Long time behavior of the two-dimensional Vlasovequation with strong external magnetic field, Math. Models Methods Appl. Sci. 10(2000) 539-553.

[21] E. Frénod, E. Sonnendrücker, The finite Larmor radius approximation, SIAM J. Math. Anal. 32(2001) 1227-1247.

[22] F. Golse, L. Saint-Raymond, The Vlasov-Poisson system with strong magnetic field, J. Math. Pures Appl. 78(1999) 791-817.

[23] D. Han-Kwan, Effect of the polarization drift in a strongly magnetized plasma, Math. Model. Numer. Anal. 46(2012) 929-947.

[24] P.-L. Lions, B. Perthame, Propagation of moments and regularity for the 3dimensional Vlasov-Poisson system, Invent. Math. 105(1991) 415-430.

[25] R.G. Littlejohn, Hamiltonian formulation of guiding center motion, Phys. Fluids 24(1981) 1730-1749.

[26] R.G. Littlejohn, Variational principles of guiding centre motion, J. Plasma Phys. 29(1983) 111-125.

[27] E. Miot, On the gyrokinetic limit for the two-dimensional Vlasov-Poisson system, arXiv:1603.04502.

[28] K. Pfaffelmoser, Global classical solutions of the Vlasov-Poisson system in 3 dimensions for general initial data, J. Differential Equations 95(1992) 281-303. 
[29] L. Saint-Raymond, The gyro-kinetic approximation for the Vlasov-Poisson system, Math. Models Methods Appl. Sci. 10(2000) 1305-1332.

[30] L. Saint-Raymond, Control of large velocities in the two-dimensional gyro-kinetic approximation, J. Math. Pures Appl. 81(2002) 379-399.

[31] T. Ukai, S. Okabe, On the classical solution in the large time of the two dimensional Vlasov equations, Osaka J. Math. 5(1978) 245-261. 